The Effects Of A Novel Endothelin Receptor Antagonist, Macitentan, On Right Ventricular Substrate Utilization And Function In A Sugen5416/Hypoxia Rat Model Of Severe Pulmonary Artery Hypertension

Katarzyna Drozd

This Thesis is Submitted as Partial Fulfillment of the Master of Science Program in Cellular and Molecular Medicine

Department of Cellular and Molecular Medicine

Faculty of Medicine

University of Ottawa

(C) Katarzyna Drozd, Ottawa, Canada, 2015 


\section{ABSTRACT \\ The Effects of a Novel Endothelin Receptor Antagonist, Macitentan, on Right Ventricular Substrate Utilization and Function in a Sugen5416/Hypoxia Rat Model of Severe Pulmonary Artery Hypertension}

Background-Pulmonary artery hypertension (PAH) is characterized by progressive vascular changes causing increased pulmonary resistance and eventual right heart failure (HF). It has been suggested that altered myocardial substrate utilization may be associated with right HF, however these changes have not yet been well characterized. The aim of this study was to evaluate in vivo right ventricular (RV) function and RV glucose and fatty acid metabolism in an experimental model of PAH using non-invasive positron emission tomography (PET) imaging and to investigate the effect of a novel endothelin receptor antagonist, Macitentan, on the development of PAH and RV energetics. Methods and Results-Severe PAH was induced in a total of 11 male SpragueDawley rats using a single injection of Sugen5416 followed by chronic hypoxia. The rats were then randomized to treatment or no treatment with Macitentan (30 $\mathrm{mg} / \mathrm{kg}$ daily) Five and eight weeks post injection, substrate utilization was serially assessed with 2$\left[{ }^{18}\right.$ F]fluoro-2-deoxyglucose (FDG) and 4-[ ${ }^{18}$ F]fluoro-6-thia-heptadecanoate (FTHA) PET scans for glucose and fatty acid metabolism respectively, and reported as a standardized uptake value (SUV). This data was correlated with in vivo functional measurements with echocardiography and multi gated acquisition scans. The Sugen-hypoxia (SuHx) model resulted in an increase in RV FDG uptake over 8 weeks (SUV control: $1.56 \pm 0.38$, week 5 SuHx: $4.06 \pm 1.90$, week 8 SuHx: $4.00 \pm 1.60, p<0.005$ between control and week 5 SuHx). RV FTHA data showed a trend towards increased uptake with onset of PAH at 
week $5 \mathrm{SuHx}$ (SUV control: $1.50 \pm 0.40$, week $5 \mathrm{SuHx}: 3.06 \pm 1.10$, p $>0.05$ ). Macitentan significantly decreased RV FDG uptake (SUV week 8 SuHx: $4.00 \pm 1.60$, week 8 SuHx +ERA: $2.54 \pm 0.90, \mathrm{p}<0.05)$. This was associated with improved RV ejection fraction (PAH week 8 untreated: $53.15 \pm 9.9 \%$ vs PAH week 8 treated: $73.22 \pm 4.8 \%, \mathrm{p}<0.01$ ) and improved pulmonary artery pressures measured by pulmonary artery acceleration time (PAH week 8 untreated: $17.32 \pm 2.30 \mathrm{~ms}$ vs. PAH week 8 treated: $24.38 \pm 3.90 \mathrm{~ms}$, $\mathrm{p}<0.001)$. There was a strong correlation between increased pulmonary artery pressures and increased RV FDG uptake $(\mathrm{r}=0.87, \mathrm{p}=0.001)$ as well as a significant inverse relationship between improved RV ejection fraction and decreased RV FDG uptake ( $\mathrm{r}=-$ 0.72, $\mathrm{p}=0.01$ ). Conclusion-PAH is associated with metabolic changes in the RV, characterized by increased glucose uptake and a trend towards increased RV fatty acid uptake with onset of PAH. Macitentan attenuated RV FDG uptake and significantly increased RV function as well as hemodynamics compared to untreated group. 


\section{ACKNOWLEDGEMENTS}

First and foremost, I would like to sincerely thank my supervisors Dr. Lisa Mielniczuk and Dr. Jean DaSilva for the opportunity to do research with you, learn from you and be mentored by you.

I have never met someone so passionate and driven, as Dr. Lisa Mielniczuk, about research and helping her patients. You have rubbed off on me in such a positive way and I will always have a place for hearts in my heart.

I would also like to thank the members of my thesis advisory committee Dr. Mary-Ellen Harper and Dr. Duncan Stewart and MFI program director Dr. Rob Beanlands. The breadth of knowledge you brought to the table has truly helped me in my research and it has been a pleasure learning from you.

Dr. Yupu Deng and Dr. Baohua Jiang have helped me with the technical aspects of my project. They conducted all the hemodynamic experiments and provided the catheterization equipment to do so.

There are many people I would like to thank that helped with the imaging portion of my project. Julia Petryk: master of micro SPECT, Christine Archer, Crystal MacDonald, Dr. Etienne Croteau and Dr. Rob DeKemp: micro PET and Marika Kolajova: echocardiography. Marika has also been instrumental in helping me gain laboratory skills and has helped me with Western Blotting. Also, thank you for always making it fun! I would also like to extend my gratitude to the staff of ACVS for providing the best care for my animals throughout the study, and being patient with my equipment difficulties. Finally, I dedicate this thesis to my family and friends! Your support was the key to my success and I appreciate you all so much. 


\section{TABLE OF CONTENTS}

ABSTRACT

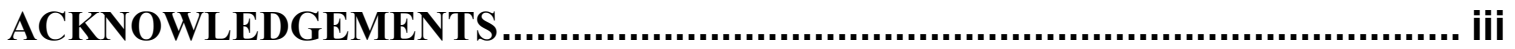

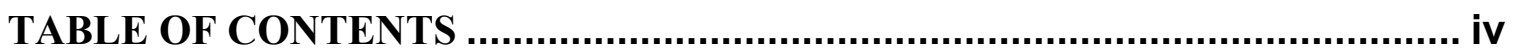

LIST OF FIGURES

LIST OF ABBREVIATIONS ......................................................................... viii

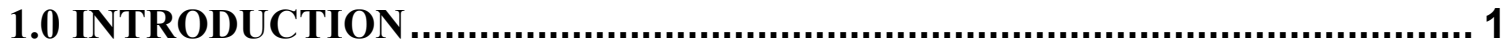

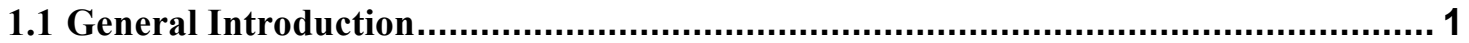

1.2 Pulmonary Artery Hypertension ..................................................................... 2

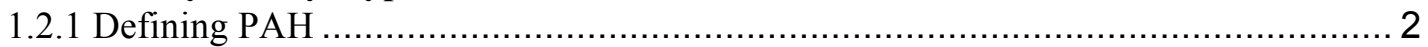

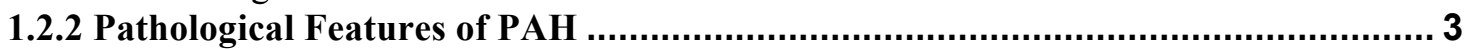

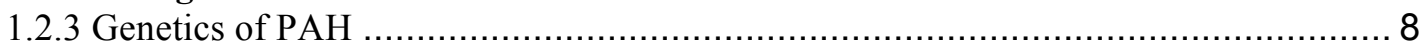

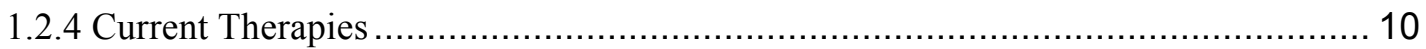

1.4 Cardiac Metabolism ............................................................................. 14

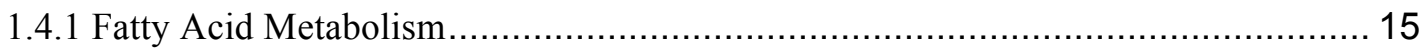

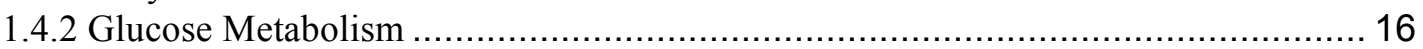

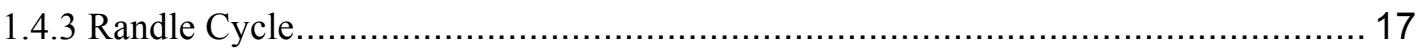

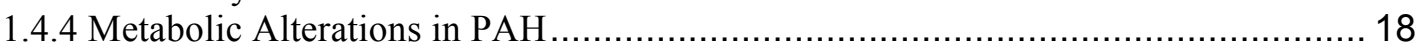

1.4.5 Metabolic Alterations in Pulmonary Vascular Cells ....................................... 19

1.4.6 Metabolic Alterations in the Right Ventricle ................................................ 20

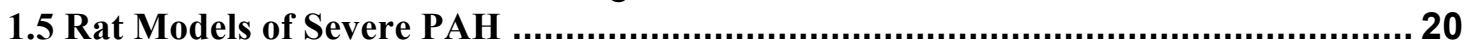

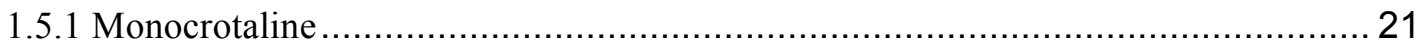

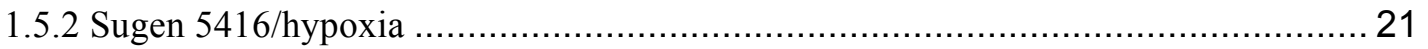

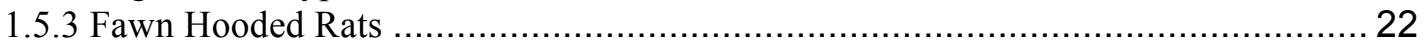

1.6 Positron Emission Tomography ....................................................................... 23

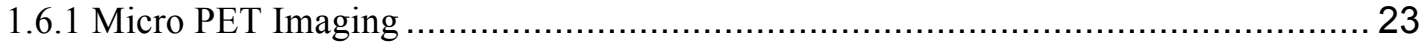

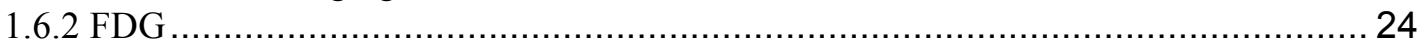

1.6.3 FTHA.

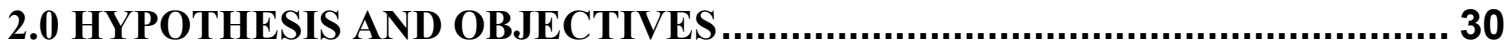

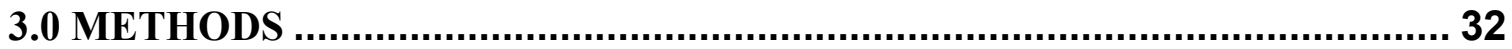

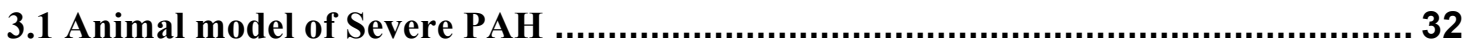

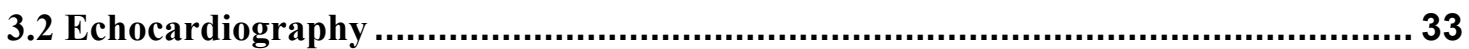

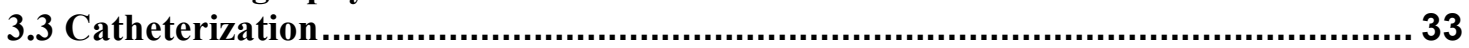

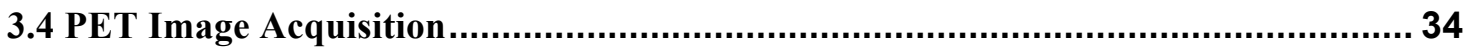

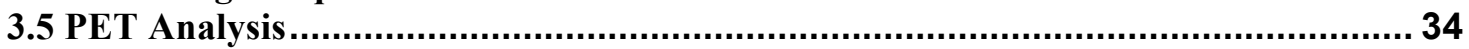

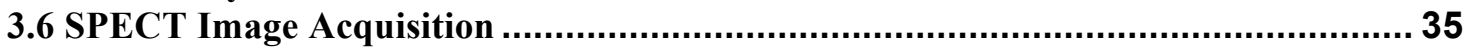

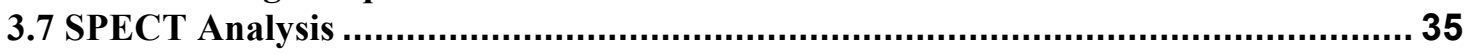

3.8 Determination of Protein Concentration ............................................................. 35

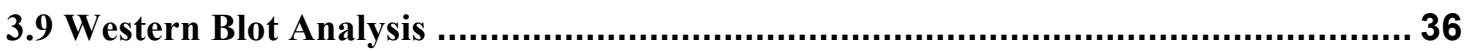

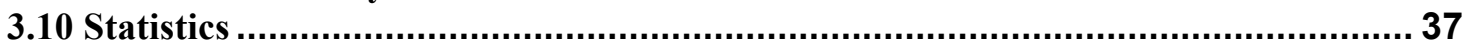




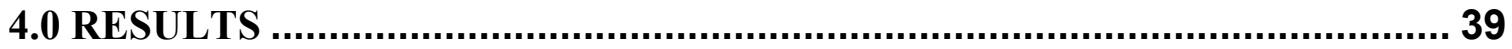

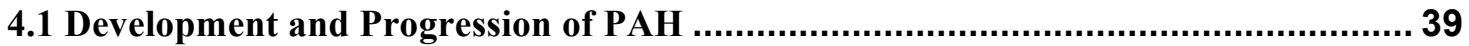

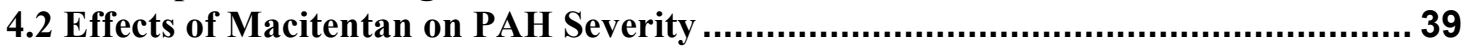

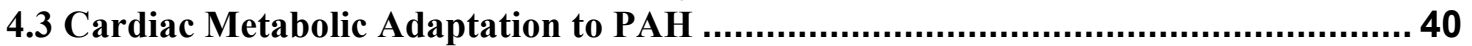

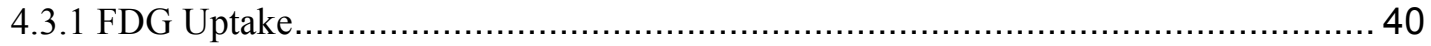

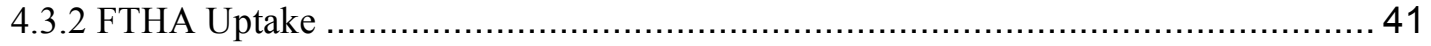

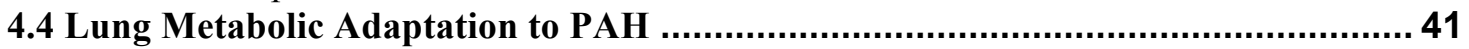

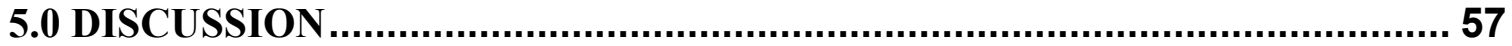

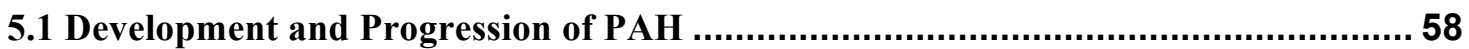

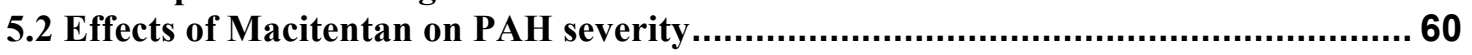

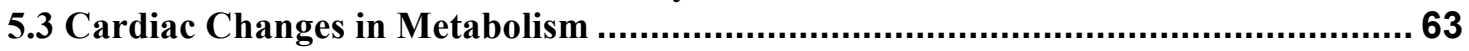

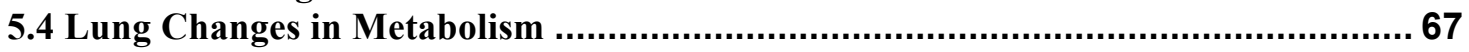

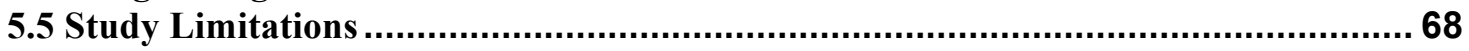

6.0 CONCLUSIONS AND FUTURE WORK ............................................. 71

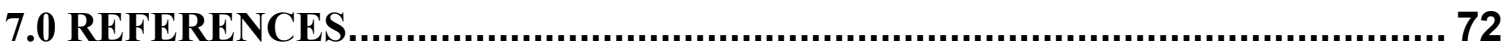




\section{LIST OF FIGURES}

Figure 1.1: Schematic Representation of the Translational Pathway from Pre-clinical Animal Studies to Clinical Research. (Page 26)

Figure 1.2: The Randle Cycle: Metabolic Relationship Between Fatty Acid Oxidation and Glucose Oxidation. (Page 27)

Figure 1.3: Annihilation Event Producing PET Signal. (Page 28)

Figure 1.4: Pet Tracers of Metabolism. (Page 29)

Figure 3.1: Experimental Protocol for the Evaluation of Endothelin Receptor Antagonist Macitentan, on Right Ventricular and Pulmonary Artery Substrate Utilization in SuHx Rodent Model of Severe PAH. (Page 38)

Figure 4.1: Development of PAH Demonstrated by Echocardiography. (Page 43)

Figure 4.2: Hemodynamic Effect of SuHx Exposed PAH Rats. (Page 44)

Figure 4.3: Measure af Right Ventricular Hypertrophy Following Macitentan Treatment. (Page 44)

Figure 4.4: Effect of Macitentan Treatment on PAAT. (Page 45)

Figure 4.5: Effect of Macitentan Treatment on hemodynamics. (Page 45)

Figure 4.6: Effect of Macitentan Treatment on RV Ejection Fraction. (Page 46)

Figure 4.7: Representative Histological and Immunohistochemical Images of Small Pulmonary Arteries at Week 8 SuHx and Following Macitentan Treatment. (Page 47)

Figure 4.8: Effect Of Macitentan Treatment on RV Hypertrophy. (Page 47)

Figure 4.9: Quantification of RV FDG Uptake Measured by PET. (Page 48)

Figure 4.10: Kinetic Analysis of Rate of Myocardial Glucose Utilization Measured in the RV. (Page 49)

Figure 4.11: Western Blot Analysis of GLUT4 in RV Tissue Normalized to GAPDH Control. (Page 50) 
Figure 4.12: Pearson Correlation Between RV FDG SUV and Pulmonary Artery Systolic Pressure (PASP). (Page 51)

Figure 4.13: Pearson Correlation Between RV FDG SUV and RV EF. (Page 51)

Figure 4.14: Quantification of LV FDG Uptake Measured by PET. (Page 52)

Figure 4.15: Quantification of RF FTHA Uptake Measured by PET. (Page 53)

Figure 4.16: Quantification of LV FTHA Uptake Measured by PET. (Page 54)

Figure 4.17: Quantification of Lung FDG Uptake Measured by PET. (Page 55)

Figure 4.18: Quantification of Lung FTHA Uptake Measured by PET. (Page 56) 


\section{LIST OF ABBREVIATIONS}

ACC - Acetyl-CoA Carboxylase

ALK-1 - Activin Receptor-like Kinase 1

AMPK - Adenosine Monophosphate Protein Kinase

ATP - Adenosine Triphosphate

BMPR2 - Bone Morphologic Protein Receptor 2

cAMP - cyclic Adenine Monophosphate

cGMP - cyclic Guanyl Monophosphate

CPT-1 - Carnitine Palmitoyl Transferase

DCA - Dichloroacetate

EC - Endothelial Cell

ECM - Extracellular Matrix

EF- Ejection Fraction

ENG - Endoglin

ERA - Endothelin Receptor Antagonist

ET-1 - Endothelin-1

ETA - Endothelin Receptor A

ETB - Endothelin Receptor B

ETC - Electron Transport Chain

FA - Fatty Acid

$\mathrm{FADH}_{2}-$ Flavin Adenine Dinucleotide

FAO - Fatty Acid Oxidation

FAT/CD36- Fatty Acid Translocase

FDG $-{ }^{18}[\mathrm{~F}]$-fluorodeoxyglucose

FHR - Fawn Hooded Rat

FTHA - ${ }^{18}[\mathrm{~F}]$-fluoro-6-thia-heptadecanoic acid

GAPDH - Glyceraldehyde 3-phosphate dehydrogenase

GLUT1 - Glucose Transporter 1

GLUT4 - Glucose Transporter 4

GO - Glucose Oxidation

HF - Heart Failure

HIF-1 $\alpha$ - Hypoxia Inducible Factor $1 \alpha$

HK - Hexokinase

Kv 1.5 - Voltage Gated Potassium Channel

LV - Left Ventricle

MCD - Malonyl CoA Decarboxylase

MCT - Monocrotaline

NADH - Nicotinamide Adenine Dinucleotide

NO - Nitric Oxide

P-ACC - Phosphorylated Acetyl-CoA Carboxylase

P-AMPK - Phosphorylated Adenosine Monophosphate Protein Kinase

PA - Pulmonary Artery 
PAAT - Pulmonary Artery Acceleration Time

PAH - Pulmonary Arterial Hypertension

PAP - Pulmonary Artery Pressure

PASMC - Pulmonary Artery Smooth Muscle Cells

PDE5 - Phosphodiesterase Type 5

PDH - Pyruvate Dehydrogenase

PDK - Pyruvate Dehydrogenase Kinase

PET - Positron Emission Tomography

PFK - Phosphofructokinase

PH - Pulmonary Hypertension

PVR - Pulmonary Vascular Resistance

RCA - Right Coronary Artery

RHF - Right Heart Failure

RV-Right Ventricle

RVH- Right Ventricular Hypertrophy

RVSP - Right Ventricular Systolic Pressure

SERT - Serotonin Transporter

sGC - Soluble Guanylate Cyclase

SMC - Smooth Muscle Cell

SNP - Single Nucleotide Polymorphism

SPECT - Single Photon Emission Computed Tomography

SuHx - Sugen 5416/ Hypoxia model

TAC - Time Activity Curve

TCA - Tricarboxylic Acid Cycle

TGF- $\beta$ - Transforming Growth Factor $\beta$

VEGF - Vascular Endothelial Growth Factor

VEGFR2 - Vascular Endothelial Growth Factor Receptor 2

WHO - World Health Organization 


\subsection{INTRODUCTION}

\subsection{General Introduction}

Pulmonary artery hypertension (PAH) is a rare and devastating disease characterized by progressive functional and structural changes to the pulmonary vasculature with the consequential result being right heart failure (RHF) (Archer et al., 2013). The mortality is high, and prognosis remains poor (Morimatsu et al., 2012; Archer et al., 2013) for patients affected by this disease and presently prescribed therapies fail to grant a significant improvement in overall patient outcome (Nicolls et al., 2012). There exists a pressing demand to better understand the pathophysiology of PAH and the contributing factors leading to the detriment of right ventricular (RV) function, to identify new therapeutic targets and accordingly develop more effective therapies. Recently, studies have revealed that metabolic perturbations may be an important mechanism of RV failure (Fang et al., 2012) and may be implicated in pathogenic pulmonary vascular remodeling (Archer et al., 2010; Marsboom et al., 2012). Additionally, metabolic modulation has been shown to have beneficial effects on RV function by therapeutically improving metabolic efficiency and enhancing glucose oxidation (GO) (Piao et al., 2010; Archer et al., 2010; Fang et al., 2012; Marsboom et al., 2012). This may provide a new treatment avenue to directly and selectively target the failing RV and improve prognosis in patients with PAH however, there have been limited studies evaluating in-vivo metabolism of the RV (Piao et al., 2010; Fang et al., 2012). Serial positron emission tomography (PET) using labeled substrates of metabolism may be used to study myocardial and lung energetics non-invasively in otherwise inaccessible 
tissues. Metabolic imaging provides the advantage of direct translation of methodology from rodent models to clinical practice and may be used as a diagnostic tool or a way to monitor response to treatment in patients with $\mathrm{PAH}$.

The Sugen5416/hypoxia ( $\mathrm{SuHx}$ ) animal model of severe PAH is a relevant model that provides the opportunity to investigate molecular mechanisms of PAH as it closely reflects the pathobiology of human PAH (Nicolls et al., 2012). This "two-hit" model exhibits severe PAH and is able to produce intimal lesions in the small pulmonary arteries (PA) that are histologically indistinguishable from the characteristic plexiform lesions found in humans (Sakao et al., 2010). The results of this study, will significantly advance our understanding of metabolic alterations and the pathogenesis of RHF; data which is currently lacking in PAH patients. In addition, this study will demonstrate a role for metabolic imaging as an in-vivo tool for the study of cardiac metabolism and has the potential for further translational study in humans. This is a vital initial step towards further research in the role of metabolic modulators in the treatment and prevention of right HF in PAH. (Figure 1.1)

\subsection{Pulmonary Artery Hypertension}

\subsubsection{Defining PAH}

Pulmonary Hypertension (PH) is a collection of complex vasculopathies that cause pressures in the arteries of the lungs to rise to dangerous levels. Recently amended by the World Health Organization (WHO), it classifies five main categories characterized by pathology and clinical presentation (McLaughlin et al., 2011). The development of a universal 
nomenclature for the grouping of different forms of $\mathrm{PH}$ has allowed better clinical evaluation and judgment for therapeutic intervention and systematic approach with better-defined groups. This has lead to the evolution of novel informative animal models for conducting research and clinical trials and development of new treatments (Humbert et al., 2004; Simonneau et al., 2008). PAH belongs to category 1 of $\mathrm{PH}$ and is further divided into 5 subsets namely idiopathic, heritable, drug- and toxic- induced, associated with connective tissue diseases and persistent pulmonary hypertension of the newborn (Simonneau et al., 2008) each sharing common hemodynamic and histological features (Ryan et al., 2014). PAH is hemodynamically defined as elevated mean pulmonary artery pressures (PAP) > $25 \mathrm{mmHg}$ at rest, increased pulmonary vascular resistance (PVR) $>3$ Wood units and a decrease in pulmonary capillary wedge pressure $<15 \mathrm{mmHg}$ and presents without underlying lung of left-heart disease (Peacock et al., 2007; Archer et al., 2010; Rabinovitch et al., 2012).

The incidence and prevalence of PAH on a global scale is difficult to assess due to a lack of clinical data and national registries on this patient population, undiagnosed or misdiagnosed cases and limited access to healthcare in developing countries (Peacock et al., 2007). Two national epidemiological studies conducted in France and Scotland have estimated an annual incidence rate of 2.4 cases/million and 7.6 cases/million respectively (Humbert et al., 2006; Peacock et al., 2007). PAH develops more frequently in females than males (2:1 ratio) and may affect individuals of any age.

\subsubsection{Pathological Features of PAH}




\subsubsection{Vascular Remodeling}

The pulmonary circulation is a low-pressure, high-flow system comprised of thin walled vessels that pass the RV output to alveolar structures in the lungs for gas exchange (Jeffery et al., 2002; Greyson et al., 2010). The blood supply is then introduced into the left side of the heart where it is pumped back into the systemic circulation, carrying oxygenated blood to various tissues. There exists a dynamic relationship between output volumes of the RV and the pressures within lungs (Greyson et al., 2010) that acts as a response buffer adapting to physiological changes. Certain pulmonary pathologies may cause persistent changes within the vasculature and increase RV afterload. The RV compensates by gaining mass in the form of hypertrophy allowing it to generate more force per contraction. However, the chronic state of stress experienced by the RV often leads to development of RHF (Greyson et al., 2010). $\mathrm{PAH}$ is a vasculopathy that affects the small PAs and is characterized by progressive structural and functional changes to the vasculature. The result is a narrowing or complete obliteration of the vessels and consequently increased PVR (Jeffery et al., 2002; Humbert et al., 2006). Changes occur in all three layers of the vessel namely the intima, media and adventitia.

Beginning with the inner most layer of the PA, there lays a single monolayer of endothelial cells (EC) responsible for the majority production and release of vasoactive agents and is involved in homeostasis, growth, differentiation and cell signaling (Galie et al., 2004; Budhiraja et al., 2004). A proposed mechanism of vascular remodeling has emerged which describes an initial apoptotic event causing an emergence of apoptosis-resistant, dysfunctional ECs (Sakao et al., 2009; Voelkel et al., 2012). With the dysfunction of ECs, comes an imbalance in production of vasoactive mediators. Production of vasodilators, nitric 
oxide (NO) and prostacyclin are decreased while vasoconstrictors thromboxane, serotonin and endothelin-1 (ET-1) are increased with the outcome favouring vasoconstriction (Archer et al., 2010; Crosswhite et al., 2014). Apart from affecting vascular tone, the endothelin releases a number of paracrine factors (Budhiraja et al., 2004; Humbert et al., 2008; Archer et al., 2010) that are important for the proliferative activity of pulmonary artery smooth muscle cells (PASMC) (Stewart et al., 1991; Farber et al., 2005). Disturbances in signaling due to dysfunctional endothelium may play a role in medial thickening observed in PAH (Stewart et al., 1991; Budhiraja et al, 2004; Humbert et al., 2008). The endothelial layer acts as a barrier between the serum and downstream vascular components and is a sensor to stimuli such as sheer stress, toxins, and signaling proteins. Exuberant EC proliferation also leads to the development of glomeruloid-like structures located at bifurcation sites of small pulmonary arteries (Jonigk et al., 2011;Voelkel et al., 2012). Commonly termed plexiform lesions, the growth of these characteristic irreversible lesions leads to vascular occlusion (Voelkel et al., 2012) and has been associated with poor prognosis in patients (Rai et al., 2008).

The next layer to be affected in pathogenic PAH is the media, comprised of vascular smooth muscle cells (SMC). In the disease state there is suppressed apoptosis, increased proliferation and muscularization of normally non-muscular distal and peripheral arteries (Humbert et al., 2004), creating a stiffened, noncompliant vessel. This phenotype is characterized by mutation or downregulation in bone morphologic protein receptor 2 (BMPR2), loss of voltage-gated potassium (Kv1.5) channels, metabolic abnormalities (Humbert et al., 2004), overexpression of serotonin transporter (5-HTT) (Guignabert et al., 2006), increased 
expression of various growth factors (Crosswhite et al., 2014) and impaired cross-talk with ECs.

The adventitia is the complex and dynamic outermost component of the vascular wall. The connective tissue provides structural integrity to the vessel and is involved in various roles such as storing and releasing regulatory molecules involved in vessel function and maintenance of vascular homeostasis (Stenmark et al., 2013). The adventitia plays the role of injury sensing and may be activated in response to hormonal, inflammatory or environmental stresses (Stenmark et al., 2013) by upregulating contractile, adhesion or extracellular matrix (ECM) proteins or releasing chemokines, cytokines or angiogenic and growth factors (Stenmark et al., 2013) that stimulate cellular responses. Adventitial changes in PAH include increased thickness and collagen deposition in response to chronic elevated intravascular pressures (Jeffery et al., 2002).

\subsubsection{Right Ventricular Dysfunction}

The consequence of the pulmonary vascular remodeling exhibited in PAH is increased RV afterload. In response to these changes, it is imperative for the RV to compensate and generate enough force to push blood through the narrowed pulmonary circulation, and this is achieved by right ventricular hypertrophy $(\mathrm{RVH})$. There is heterogeneity in the way the RV responds to chronic pressure overload, and patients may either exhibit an adaptive or maladaptive phenotype (Archer et al., 2013). Adaptive RV remodeling is associated with minimal dilatation and myocardial fibrosis and a preserved RVEF (Archer et al., 2013). In maladaptive cases there is profound RV dilatation, fibrosis and hypokinesis and steady 
deterioration of RV function (Archer et al., 2013). Interestingly both cases may display the same degree of advanced vascular remodeling, yet the clinical turnout of each case will be significantly different. Although our knowledge is limited as to what drives the RV down the maladaptive path versus adaptive, it has been suggested that metabolic adaptation may be an underlying factor (Archer et al., 2013).

An enlarged heart requires proportional increases in myocardial perfusion and oxygenation to supply the heightened demand, however studies in both patients and experimental models of PAH demonstrate the presence of RV ischemia reflected by a reduction in capillary density and impaired right coronary artery (RCA) flow (Van Wolferan et al., 2008; Archer et al., 2013). Recently, Boogard and colleagues investigated two models of PAH emulating adaptive and maladaptive RVH. An adaptive murine model of RVH is a model of chronic pressure overload and is accomplished by pulmonary artery banding (Boogard et al., 2009). The maladaptive model of RVH was induced by $\mathrm{SuHx}$ and mimicked angioproliferative PAH. The latter model was associated with a loss in microcirculation and extensive myocardial fibrosis leading to reduced RV function. The conclusion of their study was that an isolated increase in pulmonary pressures was insufficient to produce RVF and that a lack of angiogenesis to support the growth of the heart was detrimental (Boogard et al., 2009). This study is a prelude to the possibility of metabolic modulation as a therapeutic strategy, which would shift metabolism to be more efficient by yielding higher amounts of ATP with less oxygen.

RHF is the primary cause of death in patients with PAH (Drake et al., 2010). Clinical presentation of PAH includes breathlessness, syncope, weakness, angina and abdominal 
distension. Patients with deteriorating health status and development of RHF will exhibit these symptoms at rest and experience discomfort by physical activity (Galie et al., 2004). Clinically, patients with failing RV will present with reduced RV contractility, hypotension or end-organ dysfunction (Price et al., 2010). Results of a study conducted by Campo and colleagues in 2011 on outcomes of hospitalization for RHF, revealed high mortality rates and poor prognosis associated with the development of RHF in PAH patients. Excluding patients that died while in hospital, 33\% PAH patients originally hospitalized for RHF died after oneyear (Campo et al., 2011). The exceedingly high mortality rates associated with RHF are testament to the pressing need for development of new therapies that directly enhance RV function.

\subsubsection{Genetics of PAH}

The capabilities of high throughput sequencing and resulting genome-wide association studies have provided insight to the hereditary predispositions associated with PAH (Soubrier et al., 2013). Identifying genetic alterations helps pinpoint pathogenic culprits and their downstream effects, and exposes new therapeutic targets. Apart from providing molecular information, understanding the genetics behind $\mathrm{PAH}$ has created a new form of patient care with the availability of genetic testing and counseling to prevent transmission to kin.

The most common pathogenic mutation has been identified in BMPR2, a type II receptor of the transforming growth factors (TGF- $\beta$ ). The mutation is present in approximately $75 \%$ of familial cases and $25 \%$ of sporadic cases of PAH (Soubrier et al., 2013) and is associated 
with significant risk. The TGF- $\beta$ family of proteins is involved in a number of homeostatic functions and biological processes such as proliferation, differentiation, apoptosis, adhesion, migration and the production of ECM in ECs and SMCs (Pardali et al., 2012; Guo et al., 2012). The integral type II receptor binds certain ligands that activate the intracellular Smad pathway leading to the transcription of target genes (Fessel et al., 2011). The BMPR2 is also involved in a Smad-independent signaling pathway, which activates cell survival pathways (Fessel et al., 2011). TGF- $\beta$ signaling is important in vasculogenesis and angiogenesis and mutations causing improper signaling leads to a number of cardiovascular pathologies (Pardali et al., 2011) and tumorigenesis (Fessel et al., 2011). Rodent models with a homozygous deletion in BMPR2 present embryonic lethality. Additionally, engineered alterations in BMPR2 expressions have been investigated in murine models and produced many similar features to human PAH such as increased RVSP, RV dilatation, muscularization of small pulmonary arteries, and the formation of complex lesions (Fessel et al., 2013).

Although not as common as the BMPR2 mutation, other genes have been linked to the pathogenesis of PAH such as the activin receptor-like kinase 1 (ALK-1) and endoglin (ENG). Both genes are accessory receptors of the TGF- $\beta$ superfamily participating in the Smad-dependent signaling cascade, and both induce negative effects on EC function and proliferation (Nassiri et al., 2011; Pardali et al., 2011; Upton et al., 2013).

Single nucleotide polymorphism (SNP) is a genetic phenomenon described as a change in one nucleotide of a coding sequence in a gene (Archer et al., 2010). SNPS are common and occur frequently without consequence, however in some cases may affect the amino acid 
sequence of a protein and change its function. These types of variations may enhance a person's susceptibility of developing disease and may explain predisposition in PAH (Archer et al., 2010). A few SNP variants are identified to be PAH-related, including KCNA5, gene coding for Kv1.5 channels (Remillard et al., 2007) and serotonin transporters (SERT or 5HTT) (Fessel et al., 2011). Significant progress has been made in understanding genetic and molecular mechanisms of PAH. Several candidate genes involved in the regulation of vascular tone or cellular proliferation have been targets of genetic testing. However, the limited studies conducted have failed to provide confidence in statistical association as being a major risk factor for PAH and further research is warranted (Fessel et al., 2013). Identification of novel genetic alterations underlying PAH will hopefully lead to earlier diagnosis, new therapeutic targets, disease prevention and ultimately a cure (Upton et al., 2013)

\subsubsection{Current Therapies}

Current available therapies for PAH are targeted at improving the hemodynamic status of patients and at providing symptomatic relief. Disappointingly, current pharmacological options fail at significantly improving patient outcomes, reversing disease or decreasing mortality rates (Dewachter et al., 2010), and they remain expensive (Archer et al., 2010; Chakinala et al., 2013). Therefore, there exists a pressing need to explore new avenues for target therapy and to discover drugs that will directly improve RV function, which remains the main predictor of prognosis. We must also aim to better design long-term clinical trials with more clinically relevant end-points to provide better prognostic accuracy. 
There exists four classes of first-line drugs, which are available and commonly prescribed for the treatment of $\mathrm{PAH}$; prostanoids, endothelin receptor antagonists (ERA) and phosphodiesterase type 5 (PDE5) inhibitors and the newest class, soluble guanylate cyclase (sGC) stimulators (Ruan et al., 2010; Agarwal et al., 2011).

Prostanoids possess vasodilatory effects that mimic endogenous prostacyclin, which is produced by ECs and is reduced in patients with PAH (Stewart et al., 1991). Prostacyclin induces its effects by binding to G-protein coupled receptors found on the surface of ECs. This activates the G-protein and production of intracellular cyclic adenosine monophosphate (cAMP), which activates protein kinase A. In turn, the signaling cascade inhibits platelet aggregation, enables relaxation of SMCs and causes vasodilation of the PAs (Ruan et al., 2010). Treatment with prostacyclin has been challenging due to its short-half life and instability at room temperatures. However, recent chemical modifications have resulted in new analogues that may be delivered subcutaneously or as inhalants, and do not need to be refrigerated (Ruan et al., 2010). The use of prostacylin for the therapy has yielded positive effects on exercise tolerance, hemodynamics, breathing and short-term survival (Ruan et al., 2010).

PDE5 inhibitors prevent the degradation of secondary messengers, cyclic guanosine monophosphate (cGMP) and cAMP, which are induced upon signal transduction involving NO, an endogenous vasodilator. PDE5 inhibitors have shown to improve hemodynamics but also acutely improve RV contractility (Nagendran et al., 2007) 
ERAs are a class of drugs, which block the effect of circulating ET-1. ET-1 is an endogenous peptide with potent vasoconstrictive effects and is present in higher quantities in patients with PAH (Ruan et al., 2010). ET-1 binds to two main receptors; Endothelin-A (ETA) located on SMCs and Endothelin-B (ETB) located on both SMCs and ECs. The activation of ETA receptors causes vasoconstriction and proliferation of SMCs. Binding to ETB located on SMCs causes vasoconstriction but when bound to ETB on endothelial cells, the opposite effect is produced, inducing vasodilation and ET-1 clearance. Approved ERAs vary in their specificity towards the two types of receptors. Bosentan and Ambrisentan continue to be widely used and clinical studies have shown improvement in 6-minute walk test and a decrease in time to clinical worsening (Raja et al. 2008). Macitentan is a new generation ERA that has recently been approved in Canada for long-term use in PAH patients. Its benefits over Bosentan include prolonged duration of action requiring lower dosage and a good safety profile reducing complications such a liver damage (Kunita-Takanezaw, 2014).

Soluble guanylate cyclase is an enzyme involved in the NO pathway that stimulates the production of cGMP, which causes vasodilation and inhibits SMC proliferation (Stasch et al., 2011; Lang et al., 2012). Preclinical and clinical evidence shows the benefits of sGC agonists on ameliorating hemodynamic parameters with a good safety profile (Stasch et al., 2011; Lang et al., 2012). The increasing interest for developing new classes of drugs is driven by the number of patients that do not respond to current treatments (Stasch et al., 2011). Riociguat is the first sGC stimulator to enter into phase III clinical trials and clinical development. (Stasch et al., 2011). 
Often PAH patients will receive combination therapy of two or more of the drug classes listed above. Due to the heterogeneity and progressive nature of the disease, combination therapy provides clinical stability and synergizes optimal hemodynamic and functional status (Agarwal et al., 2011). Long-term trials that determine efficacy and proper criteria for treating patients with combination therapy are undergoing (Agarwal et al., 2011).

In addition to first-line therapies, adjuvant treatment is commonly provided for symptomatic relief. Therapies such a diuretics, supplemental oxygen, anticoagulants and digoxin are often prescribed. However due to lack of long-term data, the benefits they offer for PAH patients are not fully understood (Agarwal et al., 2011)

The result of current pharmacological treatment for PAH remains unsatisfactory and available treatments do not yield curative outcomes. Providing mainly vasodilator effects, they fail to regress the complex changes that occur in the pulmonary vasculature or halt the development of RHF (Morrell et al., 2013). It is indisputable that new targets for therapy are needed to make a significant impact on patient survival. Moving forward, it is of high interest to search for agents that will reverse pulmonary vascular remodeling and directly target RV function (Dewachter et al., 2010; Archer et al., 2010).

Vascular remodeling in PAH shares many features with cancer. The cancer paradigm has prompted investigators to take a lesson on treatment strategies from the cancer model and target abnormal proliferation and impaired apoptosis. A few agents previously used for the treatment of cancer have crossed over into investigational studies for treatment of PAH including tyrosine kinase inhibitors, growth factor receptor inhibitors, elastase inhibitors, 
statins, dichloroacetate, and immunosupressants (Rai et al., 2008). The premise of using these drugs are to block cellular proliferation and induce apoptosis, which would regress vascular remodeling and lower PVR (Morrell et al., 2013).

Despite the importance of RV function to survival, the basis for RV failure remains poorly understood and this area of research warrants intensive investigation. Discovering new targets that directly and selectively improve RV function will have a significant impact on the treatment and survival outcome of patients with PAH. Metabolic modulation may serve as a tool for the treatment of RHF and preliminary studies have supported this hypothesis (McMurtry et al., 2004; Nagendran et al., 2007; Sutendra et al., 2010) The premise is to target mitochondrial/metabolic alterations in pulmonary vascular cells and myocytes. The abnormalities that occur in ECs and PASMCs include a glycolytic shift accompanied by an activation of glycolytic transcription factor hypoxia inducible factor (HIF-1 $\alpha$ ), bringing forth a growth advantage and hyperpolarized mitochondria creating apoptosis resistant cells. RV myocytes also adopt this altered phenotype however the initiation event is speculated to be ischemia driven, and the result of this shift is reduced RV contractility and hypokinesis (Morrell et al., 2013).

\subsection{Cardiac Metabolism}

The heart acts as a pump that circulates oxygen and nutrient-rich blood throughout the body to nourish various tissues. The heart has a very high-energy demand to meet the requirements and this is demonstrated by its ability to completely turnover its adenosine triphosphate (ATP) pool approximately every 10 seconds (Lopaschuk et al., 2010). Therefore, the myocardium requires ample ATP to fuel its powerhouse functions and to maintain ion 
homeostasis and basal metabolic functions (Lopaschuk et al., 2010). The heart obtains over 95\% of its ATP through the oxidative phosphorylation of fatty acids (FA) and glucose with the remainder coming from glycolysis (Lopaschuk et al., 2010).

\subsubsection{Fatty Acid Metabolism}

The adult myocardium obtains the majority of its fuel (60-90\%) through the $\beta$-oxidation of FAs. The flux of FAs through this catabolic cycle yields the greatest amount of ATP compared to other carbon substrates, however in comparison to glucose metabolism, utilizes $12 \%$ more oxygen in the process. FAs enter the myocyte by either passive diffusion or by protein-mediated transport, with fatty acid translocase (FAT)/CD36 being the major carrier. Once inside the cytoplasm, the FAs are converted into long-chain acyl CoA esters with a fate of being used for synthesis of other lipid molecules or entering the mitochondria for further metabolism. Carnitine palmitoyl transferase (CPT-1) is the gatekeeper that catalyzes the long-chain acyl CoA into long-chain acyl carnitine, which is then shuttled by the protein into the mitochondria, the site of fatty acid oxidation (FAO). One cycle of $\beta$-oxidation shortens the original FA substrate by 2 carbons and yields acetyl-CoA and two reducing equivalents flavin adenine dinucleotide $\left(\mathrm{FADH}_{2}\right)$ and nicotinamide adenine dinucleotide (NADH), supplying the tricarboxylic cycle (TCA) and electron transport chain (ETC) respectively.

\subsubsection{Regulating FAO}

FAO is regulated at the rate-limiting step, which is the shuttling of FAs into the mitochondria by CPT-1. The activity of CPT-1 is inhibited by malonyl CoA, which is synthesized from acetyl CoA via acetyl CoA carboxylase (ACC) and is degraded by malonyl CoA 
decarboxylase (MCD) (Lopaschuk et al., 2006). Therefore concentrations of intracellular malonyl-CoA and rate of FAO are governed by the balance of synthesis via ACC and degradation via MCD. The activity of ACC is regulated by AMP-activated protein kinase (AMPK), which phosphorylates the enzyme and renders it inactive. This results in a reduced rate of intracellular malonyl-CoA production and relieves FAO inhibition (Lopaschuk et al., 2006). AMPK gauges the need for fuel and responds to changes in energy demand by increase or decreasing ACC activity and secondarily the production of malonyl-CoA (Lopaschuk et al., 2010). During episodes of ischemia, there is an activation of AMPK and subsequently an increase in FAO. Although overall myocardial oxidative metabolism is reduced, FAs dominate as the main substrate source for residual oxidative phosphorylation (Fang et al., 2012). Myocardial FA metabolism is accelerated (Obrzut et al., 2010) as a compensatory mechanism to generate more fuel and inhibit apoptosis when exposed to ischemic stress however, sustained accelerated FAO rates inhibit glucose oxidation and reduce cardiac efficiency (Dyck et al., 2006).

\subsubsection{Glucose Metabolism}

The complete metabolism of a glucose molecule is achieved by two pathways; cytoplasmic glycolysis which does not require oxygen, and mitochondrial GO. Glucose molecules enter cells via facilitated transport. There are two major isoforms of glucose transporters in the myocardium; insulin-independent GLUT1 and insulin-dependent GLUT4 (Kolwicz Jr. et al., 2011). Once in the cell, glucose may either be stored as glycogen or proceed through the glycolytic pathway and be broken down for energy. The product of glycolysis results in 2 ATP molecules and pyruvate, which may be further metabolized by the TCA cycle or 
conversely converted to lactate. Before entering the TCA cycle, pyruvate is converted into Acetyl-CoA by the enzyme pyruvate dehydrogenase (PDH) and this marks the rate-limiting step of glucose metabolism.

\subsubsection{Regulating Glucose Metabolism}

The conversion of pyruvate to acetyl CoA prior to entering the TCA cycle is an important step that couples the glycolytic and GO pathways. PDH is regulated by inhibition via pyruvate dehydrogenase kinase (PDK). The upregulation of PDK is one cause of impaired GO and mechanical RV function (Archer et al. 2013). HIF-1 $\alpha$ is involved in the regulation of GO by increasing the activity/expression of PDK through a feedback loop (Michelakis et al., 2014).

Product molecules from $\beta$-oxidation namely citrate and acetyl-CoA play a role in regulating GO. Citrate inhibits the glycolytic enzyme phosphofructokinase, which causes an increase in intracellular glucose-6-phosphate, which subsequently inhibits another glycolytic enzyme, fructokinase (Archer et al., 2013). Another level of regulation is exhibited by the inhibition of PDH by acetyl-CoA yielded from FAO. In both scenarios pyruvate production is decreased and GO is impaired (Archer et al., 2013). (Figure 1.2)

\subsubsection{Randle Cycle}

The Randle cycle is a reciprocal relationship between FAO and GO whereby increases in one, inhibit the other (Hue et al., 2009; Sutendra et al., 2010) Although FAO is the major source of energy production in the adult heart, it utilizes approximately $12 \%$ more oxygen per ATP molecule produced which is significant in the context of myocardial ischemia and 
myocardial hypertrophy (Lopaschuk et al., 2006; Archer et al., 2013). It has been suggested that stimulating GO via FAO inhibition may be an effective pharmacological approach to improve cardiac function and efficiency, however a limited number of studies have thoroughly studied this in animal PAH models (Ussher et al., 2009; Sutendra et al., 2010; Piao et al., 2010; Marsboom et al., 2012). The Randle cycle may be exploited therapeutically by shifting the primary fuel source from FAs and stimulating GO. The premise of rebalancing metabolism by enhancing $\mathrm{GO}$ is to prevent mitochondrial remodeling, decrease the proliferation/apoptosis ratio and reverse pulmonary vascular remodeling. Shifting metabolism towards GO would increase metabolic efficiency in myocytes and improve RV contractility (Archer et al., 2013).

\subsubsection{Metabolic Alterations in PAH}

Recent studies have identified altered myocardial and lung energy substrate metabolism as a feature of PAH that may be associated with RHF (Humbert et al., 2004; Archer et al., 2013). These metabolic alterations include an increased dependence on ATP production via the glycolytic pathway even in an environment of plentiful oxygen, known as the "Warburg effect"(Humbert et al., 2004). Parallel with cancer pathology, increased glycolysis renders an environment that favors rapid cell proliferation and apoptosis-resistant cells (Sutendra et al., 2010; Guignabert et al., 2013; Archer et al., 2013) In PAH pulmonary vascular cells and RV myocytes exhibit this phenotype. 


\subsubsection{Metabolic Alterations in Pulmonary Vascular Cells}

Impaired glucose metabolism has been described in SMCs and ECs in both humans and rodent models of PAH (Bonnet et al., 2006; Fijalkowska et al., 2010; Xu et al., 2007). There are many pathological implications of this phenotype; 1) suppressed apoptosis, 2) impaired signaling of pro-proliferative downstream targets (ie. HIF-1 $\alpha$ and $\mathrm{Kv}$ channels) 3) accumulation of non-oxidized carbon substrates 4) mitochondrial activation of inflammatory cytokines (Michelakis et al., 2013). The metabolic remodeling exhibited in PAH vascular cells is initiated by PDK-mediated inhibition of PDH (Michelakis et al., 2013). A key molecular contributor is HIF-1 $\alpha$, which activates the transcription of pro-glycolytic enzymes such as GLUT1 and PDK (Archer et al., 2010).

The important role that PDH plays in the context of pathological PAH is demonstrated by studies that restore its function using Dichloroacetate (DCA). This small molecule binds to all 4 isoforms of PDK and inhibits its phosphorylating activity, thus preventing it from inactivating $\mathrm{PDH}$. It has been shown to reverse remodeling in PASMC by shifting the proliferation/apoptosis ratio with a net effect of apoptosis, and upregulating Kv1.5 channels in this vascular layer (Michelakis et al., 2002; McMurtry et al., 2004; Sutendra et al., 2010). The use of metabolic modulator, DCA, for treatment of PAH has shown promising results in animal models (McMurtry et al., 2004; Bonnet et al., 2006). Additionally, DCA has been used as a therapy for metabolic disease and lactic acidosis, which lowers the barrier for clinical trials and translation into PAH patients (Archer et al., 2010; Michelakis et al., 2013). 


\subsubsection{Metabolic Alterations in the Right Ventricle}

The glycolytic phenotype is also present in RV myocytes in PAH. In response to increased pulmonary resistance the RV undergoes hypertrophy to allow it to generate more force to move blood through the tightened vessels. The increase stress exhibited by the RV is associated with myocyte death, increased fibrosis and presence of non-contractile proteins resulting in contractile dysfunction and heart failure (Michelakis et al., 2013). As an initial compensatory mechanism and response to ischemia, the myocytes switch to glycolysis to grow in size and prevent apoptosis. Long-term however, this phenotype has negative effects on cardiac function. Glycolysis is insufficient at producing enough energy to maintain proper contractile function of the RV myocytes. Accelerated flux through this pathway also leads to increased lactate production and an uncoupling between glycolysis and glucose oxidation causing increases in proton production. The RV is then forced to redirect its energy to reestablish ion homeostasis rather than expedite its fuel for contractile function.

\subsection{Rat Models of Severe PAH}

In order to investigate mechanisms of disease or novel pharmacological agents, an appropriate animal model may be used as a surrogate before entering the human candidate. Animal models should closely reflect the pathobiology of the disease being studied. It is doubtful that there will ever be a model that perfectly depicts the human disease and therefore preclinical data must be carefully analyzed and interpreted (Archer et al., 2010). Despite this, preclinical animal studies have provided useful insight into molecular mechanisms of disease and continue to advance our knowledge of PAH. 


\subsubsection{Monocrotaline}

Monocrotaline (MCT)-induced PAH is one of the oldest and most widely used models of PAH (Stenmark et al, 2009). MCT is a naturally occurring pyrrolizidine alkaloid toxin found extracted from the plant species Crotalaria (Nishimura et al, 2002; Stenmark et al., 2009). PAH is induced in rats by administering one single injection of the toxin, making it affordable and technically simple (Stenmark et al., 2009). The exact mechanism by which MCT induces PAH is unclear however, it has been suggested that direct endothelial damage is the initiating event (Stenmark et al, 2009). MCT causes an increase in PAP and vascular remodeling characterized by disorganized endothelial proliferation and medial hypertrophy (Stenmark et al., 2009). The model also produces significant RV hypertrophy and RV dysfunction. However, there are problems associated with this model. The degree of severity of PAH that manifests varies significantly between strains, species and animals due to differences in liver enzymes that metabolize MCT making the model unpredictable (Stenmark et al., 2009). MCT also produces systemic effects, causing injury to other tissues, including kidney and liver (Roth et al., 1981). It is interesting to point out that over 30 therapeutic agents have been tested in MCT rat model and all treatments prevented, reversed or cured PAH (Stenmark et al., 2009). The MCT model also does not develop complex plexiform lesions, a hallmark histological feature of human PAH. This suggests that perhaps the MCT model does not exhibit the complex pathobiology that is present in human PAH.

\subsubsection{Sugen 5416/hypoxia}

A common approach of inducing severe, irreversible PAH in rats is a single subcutaneous injection of SUGEN5416, a potent vascular endothelial growth factor receptor 2 (VEGFR2) 
inhibitor, combined with 3 weeks exposure to chronic hypoxia (10\% oxygen), followed by 2 weeks re-exposure to normoxia (21\% oxygen). First described by Taraseviciene-Stewart and colleagues (Taraseviciene-Stewart et al., 2001), this model is premised on the involvement of vascular endothelial growth factor (VEGF) in the proper maintenance and differentiation of ECs. This two-hit approach triggers endothelial dysfunction and selection of an apoptosis resistant cellular phenotype (Humbert et al., 2004). Contrast to other PAH rodent models, the SuHx model closely mimics the hemodynamic and histological features found in severe human idiopathic PAH (Humbert et al., 2004; Abe et al., 2010). Among these features is the formation of complex plexiform lesions in the pulmonary vasculature that are histologically analogous to those found in human arteriopathy (Abe et al., 2010). The irreversible vascular remodeling and sustained high pressures cause RV failure in this model, making it appropriate to study the basis and development of RVF (Sakao et al., 2010). Another advantage to using this model is its lung specificity. The drug combination with hypoxia only affects lung vasculature and not other tissues (Sakao et al., 2010).

\subsubsection{Fawn Hooded Rats}

The Fawn-Hooded rat (FHR) strain develops PAH spontaneously (Archer et al., 2011). Due to its predisposition, this model is regarded as informative to study the inheritable form of PAH (Van Genechten et al., 2003). FHR develops PAH following a short exposure to mild hypoxia that would have no effect in a normal animal. The structural changes within the pulmonary vasculature exhibited by this model include intimal fibrosis, medial hypertrophy and SMC proliferation leading to narrowing of arterioles and a poor prognosis (Van Genechten et al., 2003; Archer et al., 2010). 


\subsection{Positron Emission Tomography}

Positron Emission tomography (PET) is an imaging modality that is used to assess biological processes in vivo by dynamic visualization and quantification of radiolabeled tracer distribution within the body. Radiotracers are produced using a cyclotron, which undergoes a nuclear reaction that yields a neutron-deficient, unstable isotope. The isotope is then "substituted" for its non-radioactive counterpart within a target molecule. After administering the radiolabelled tracer into patient or animal subject, it will be distributed throughout the body dependent on its pharmacodynamic properties. The unstable isotope will undergo $\beta$-decay releasing a positron that will travel a short distance before colliding with nearby electrons. The annihilation event emits two antiparallel $180^{\circ}$ gamma rays of equal energy $(511 \mathrm{keV})$ that will hit a ring of detectors, which register coincident events and store them as electrical signals. (Figure 1.3) These signals get amplified, and through a series of iterative reconstructions and corrections produce a 3-dimensional dynamic picture of radiotracer distribution over time.

\subsubsection{Micro PET Imaging}

PET has good sensitivity and resolution making it applicable for investigating molecular mechanisms of disease in rodent models. Small animal PET provides a means of serially detecting and tracking changes in myocardial substrate uptake throughout the progression of PAH (Hagan et al., 2011) Positron emitting tracers designed to be analogous to substrates of cardiac metabolism, namely glucose and long-chain FAs, may be traced as they enter cellular structures and begin catabolism. Applying what we know about the tracer's kinetics, we can 
obtain a picture of myocardial metabolic activity. Kinetic analysis of tracer uptake between 10-40 minutes of a 60-minute dynamic scan allows us to measure overall influx rate $\left(\mathrm{K}_{\mathrm{i}}\right)$ into a selected tissue region. The rate of myocardial glucose utilization may be calculated by multiplying this rate constant by blood glucose concentration.

\subsubsection{FDG}

$\left[{ }^{18} \mathrm{~F}\right]$-fluorodeoxyglucose (FDG) (Figure 1.4) is a PET tracer analog of exogenous glucose labeled with ${ }^{18}$ flourine positron emitting isotope in place of the 2hydroxy group. FDG is taken up by living cells via glucose transports and enters the initial stages of the glycolytic pathway. The analog undergoes phosphorylation and the product, ${ }^{18}$ FDG-6-phosphate, cannot be further metabolized and is trapped within the cell. FDG uptake may be a measure of metabolic activity that uses glucose as a substrate, but does not distinguish between glycolysis and glucose oxidation. FDG imaging may be used as a clinical tool to monitor the right ventricle and lungs throughout the development of PAH and. Understanding how quantitative RV FDG data correlates with cardiac dysfunction may allow for better clinical management of PAH in patients.

\subsubsection{FTHA}

4- $\left[{ }^{18} \mathrm{~F}\right]$ fluoro-6-thia-heptadecanoate (FTHA) (Figure 1.4) is a long chain fatty acid analog and useful PET probe for myocardial FAO. The tracer undergoes metabolic trapping after entering $\beta$-oxidation and its uptake is proportional to the rate of myocardial fatty acid utilization. Metabolic imaging using FTHA has great potential for exploring molecular 
mechanisms involved in the pathogenesis of PAH. Future anticipated applications of FTHA would be as a clinical PET probe for diagnosis, prognosis or as a way to monitor therapeutic progress. 


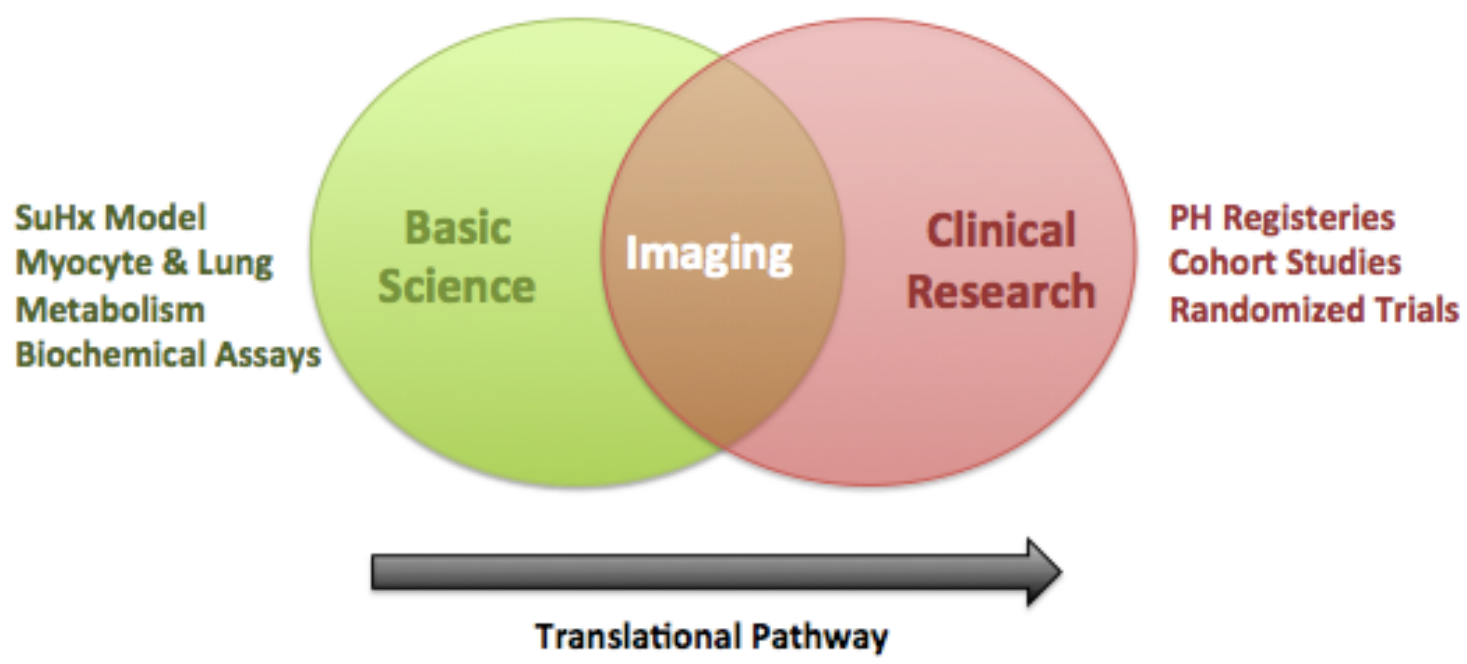

Figure 1.1: Schematic Representation of the Translational Pathway from Preclinical Animal Studies to Clinical Research. Imaging modalities such as PET provide the gateway from bench to bedside. Metabolic imaging provides the advantage of direct translation of methodology from rodent models to clinical practice and may be used as a diagnostic tool or a way to monitor response to treatment in patients with $\mathrm{PAH}$. 


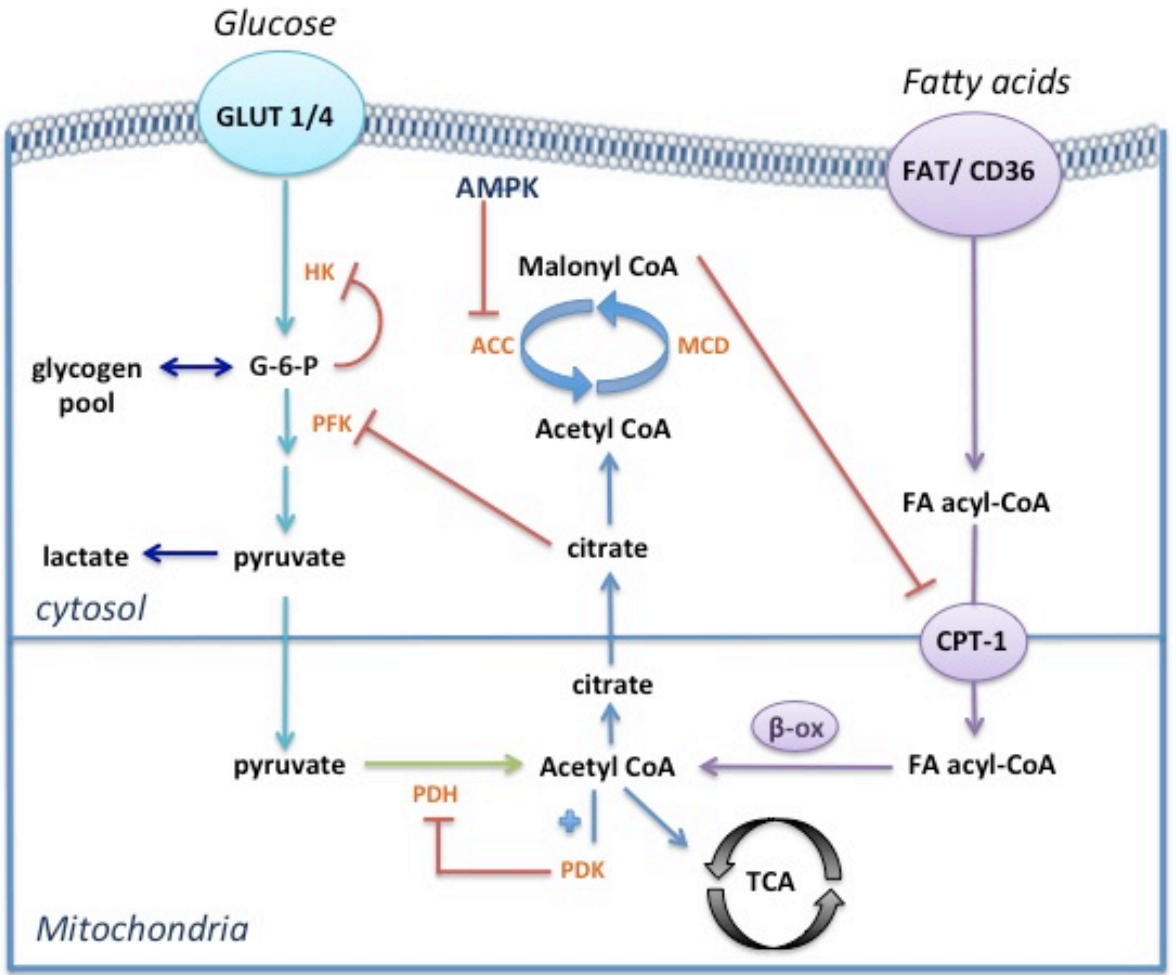

Figure 1.2: The Randle cycle: metabolic relationship between fatty acid oxidation and glucose oxidation. Acetyl $\mathrm{CoA}$ and citrate, products of $\beta$-oxidation, inhibit glycolytic enzymes phosphofructokinase (PFK) and PDH leading to a reduction in glucose oxidation. Increased intracellular Malonyl-CoA inhibits CPT-1 the gatekeeper for fatty acid oxidation. Exploiting the Randle cycle and therapeutically modulating metabolism may serve as a potential avenue for the treatment of PAH and RHF. 


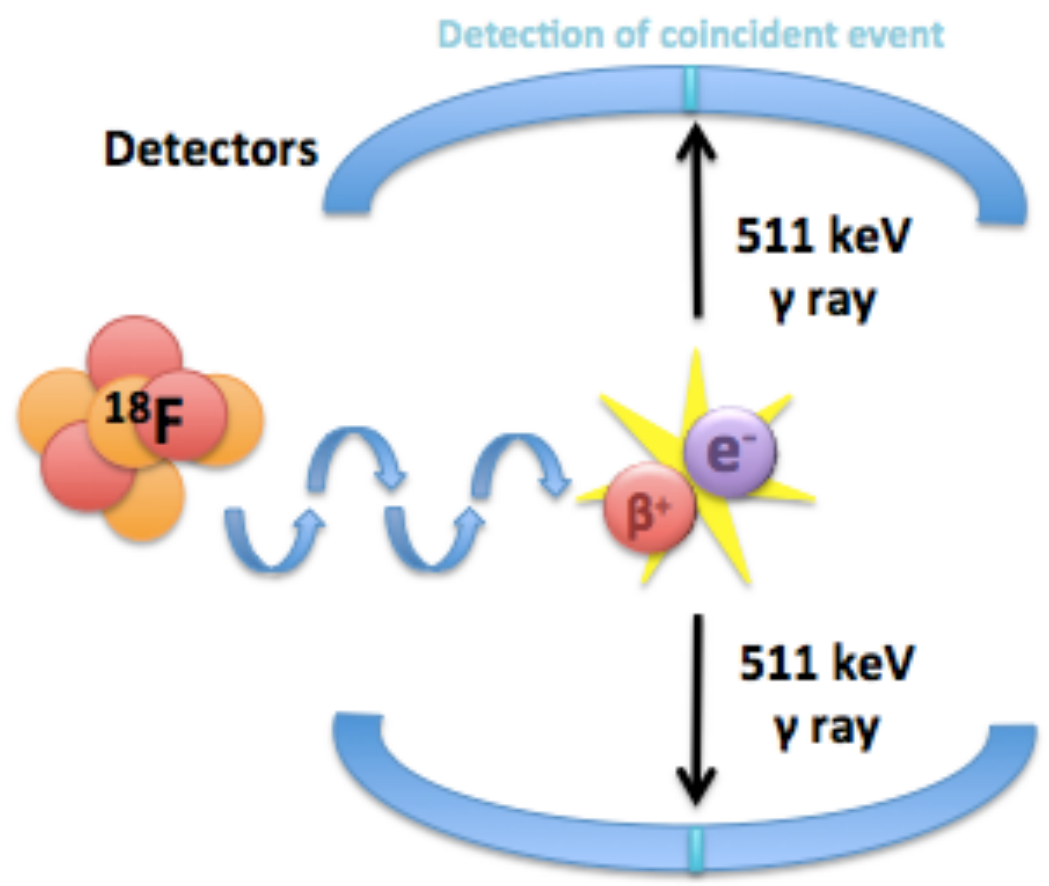

Figure 1.3: Annihilation event producing PET signal. Radiolabelled isotope undergoes beta decay emitting a positron that travels a distance and collides with an electron producing two gamma rays of equal energy $(511 \mathrm{keV})$. Surrounding detectors will detect the photons in time coincidence, which provides spatial localization. The collection of coincident events is reconstructed into a 3-dimensional image that allows for the visualization of tracer concentrations in the tissue of interest over time. 
A.

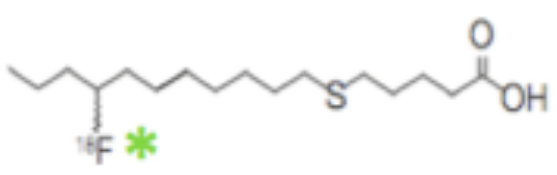

14(R,S)- $\left[{ }^{18} \mathrm{~F}\right]$ fluoro-6-thia-heptadecanoic acid (FTHA)
B.

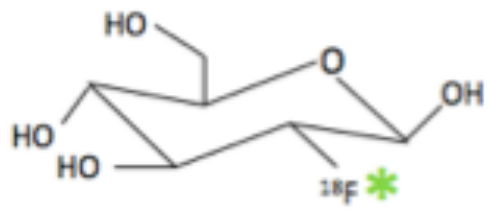

2-deoxy-2 [ $\left.{ }^{18} \mathrm{~F}\right]$ fluoro-glucose (FDG)

Figure 1.4: PET tracers of metabolism. A) FTHA is a long chain fatty acid analog that enters cells and is metabolically trapped in $\beta$-oxidation. The uptake of FTHA may be used as a measure of cellular fatty acid utilization. B) FDG is a glucose analog that is trapped after undergoing the first steps of glycolysis. FDG uptake is a measure of glucose uptake by a cell but does not distinguish between glycolysis and glucose oxidation. Asterisk (*) denotes placement of radioactive isotope. 


\subsection{HYPOTHESIS AND OBJECTIVES}

The primary hypothesis is that PAH will be associated with increased RV fatty acid and RV glucose uptake measured serially and non-invasively using small animal PET, which will be associated with pulmonary vascular remodeling and a decline in right heart function. In addition, it is hypothesized that Endothelin Receptor Antagonist therapy using Macitentan will attenuate these metabolic and functional changes.

\section{Aims}

The overall objective of this project is to evaluate the relationship between altered cardiac metabolism and the pathophysiology of PAH in the Sugen5416/hypoxia rat model of severe PAH serially using small animal PET. The specific aims of the study are as follows:

Aim 1. Determine the sequence of $R V$ metabolic changes with serial micro-PET imaging using FDG and FTHA tracer in the development of severe PAH.

1a. Correlate RV metabolic changes with RV hemodynamics.

1b. Evaluate the relationship between RV metabolic changes and RV function using echocardiography.

1c. Determine the relationship between RV metabolic changes and structural changes using RV mass. 
1d. Evaluate the relationship between PET imaging of RV metabolism and other markers of cardiac metabolism.

\section{Aim 2. Determine the sequence of lung metabolic changes with serial micro-PET imaging using FDG and FTHA tracer in the development of severe PAH}

2a. Determine if there is a relationship between lung imaging of glucose and fatty acid metabolism and pulmonary vascular remodeling.

2b. Determine the relationship between pulmonary vascular changes and the development of RV failure.

\section{Aim 3. Investigate the effect of Macitentan therapy on development of PAH and RV energetics.}

3a. Determine if Macitentan therapy is associated with a metabolic shift towards control levels using FDG PET and FTHA PET.

3b. Determine if Macitentan therapy results in regression of pulmonary vascular changes.

3c. Evaluate if Macitentan therapy is associated with improved RV function using echocardiography and multi gated acquisition scans. 


\subsection{METHODS}

A total of 40 male Sprague-Dawley rats (Charles River, Montreal, Canada) weighing 150 to $175 \mathrm{~g}$ were used for this study. Upon arrival, the rats were placed under quarantine for a 1week period of acclimatization. For the duration of the study, all rats were on a 12-hour light/dark cycle and were given standard rat chow and water ad libitum. All experimental protocols were conducted in accordance with Guide for Care and Use of Laboratory Animals and with the approval of the University of Ottawa Care and Use committee.

\subsection{Animal model of Severe PAH}

The rats were administered analgesic one hour before injection. Sugen5416 (20mg/kg, Tocris bioscience, MN, USA) was administered subcutaneously with an $18 \mathrm{G}$ needle. The rats (2 animals/cage) were immediately placed in the hypoxia chamber (coy labs, MI, USA) set at $10 \%$ oxygen for 3 weeks. After 3 weeks, animals were returned to normoxic conditions at $21 \%$ oxygen for 2 weeks. After 5 weeks post Sugen5416 injection, the rats were randomly divided into two groups: i) untreated group and ii) treated group. A control group of healthy rats was used in parallel. Treatment group animals were given a daily dose of Macitentan (30mg/kg, Actelion, Allschwil, Switzerland) mixed in a small amount of peanut butter. (Figure 3.1) 


\subsection{Echocardiography}

Transthoracic Pulsed-wave Dopplar imaging was performed in rats at 5 weeks and 8 weeks post Sugen5416 injection using Vevo 770 high-resolution imaging system (VisualSonics, Toronto, Ontario) with a 7-MHz transducer. Rats were placed on a heating pad and anaesthetized using isoflorane gas anesthesia ( $2 \%$ in oxygen). Chest and stomach hair was removed. The transducer was aligned to show pulmonary artery in the parasternal view. The sample volume was placed at $10 \%$ angle aligned with laminar flow and pulmonary outflow was recorded. Pulmonary artery acceleration time was measured using recorded pulmonary artery waveform from the time of onset of systolic flow to peak outflow velocity. Final measurements in each rat represent the sum of three measurements using manufacturer software (Vevo 770 3.0.0, Visual Sonics).

\subsection{Catheterization}

Rats were placed on a heating pad after being anesthetized with ketamine/xylazine (50:50 $1 \mathrm{~mL} / \mathrm{kg}$ IP). A small incision was made to expose the right jugular vein into which a polyvinyl catheter (Sciscense, London, Canada) was inserted and threaded through into the right ventricle for measurement of right ventricular systolic pressure. The signals were continually recorded by Sciences iWorx blood pressure system and produced measurements of RV systolic pressure. After hemodynamic assessment, each rat was euthanized by exsanguation. Hearts and lungs were then dissected for histological and immunohistochemical evaluation or for $\mathrm{RV} / \mathrm{LV}+$ Septum weight ratio measurement. 


\subsection{PET Image Acquisition}

Rats were imaged at 5 weeks and 8 weeks post Sugen5416 injection. Cardiac and lung glucose uptake and fatty acid utilization was measured in vivo in rats using FDG and FTHA tracer respectively. Rats were placed on a heating pad and anaesthetized using isoflorane gas anesthesia ( $2 \%$ in oxygen). A $26 \mathrm{G}$ catheter was placed into the tail vein and $600 \mathrm{ul}$ of blood was collected for serum glucose quantification. Animals were placed in the Siemens Inveon $^{\mathrm{TM}}$ small animal PET scanner (Siemens, Knoxville, TN, USA; $12.7 \mathrm{~cm}$ axial field-ofview, spatial resolution $<1.4 \mathrm{v} \mathrm{mm}$ ) with heart and lungs centered in the field of view. Heart rate, oxygen, body temperature, and bed temperature were constantly measured and recorded throughout the duration of the scan. A 10-minute transmission scan was acquired for anatomical reference prior to tracer injection. $30-40 \mathrm{MBq}$ of tracer was injected (0.5-1.0 ml) using a pump through the tail vein and data was acquired for 60 minutes. PET images were reconstructed using the vendor OSEM3D/MAP algorithm with 2/18 iterations respectively, into a 3D matrix of $0.31 \times 0.31 \times 0.80 \mathrm{~mm}$ voxels.

\subsection{PET Analysis}

All reconstructed dynamic PET images were quantitatively analyzed using Siemens IRW software. Regions of interest were defined to encompass the RV free wall, LV free wall, LV blood pool and lung parenchyma and threshold level was set to $50 \%$. The sampled regions were applied to all frames within the 60 -minute scan to generate representative myocardial time-activity curves of standardized uptake values over time. The image derived LV blood

pool TAC was used as an estimated input function to produce a Patlak plot, with the slope being equal to organ uptake rates (Patlak $\left.\mathrm{K}_{\mathrm{i}}\right)$. 


\subsection{SPECT Image Acquisition}

Rats were imaged at 5 weeks and 8 weeks post Sugen5416 injection. Myocardial perfusion was measured in vivo in rats using Tc-99m sestamibi blood flow tracer. Rats were placed on a heating pad and anaesthetized using isoflorane gas anesthesia ( $2 \%$ in oxygen). Rats were injected with $0.05 \mathrm{~mL}$ stannous gluceptate (IV) followed by a 30-minute incubation period. Approximately $2 \mathrm{mCi}$ of $\mathrm{Tc} 99 \mathrm{~m}-\mathrm{NaTcO} 4$ (IV) tracer was injected $(0.5 \mathrm{ml})$ and the animals were immediately imaged. Four sequential 15-minute scans were acquired using a BioScan nanoSPECT-CT scanner. SPECT images were reconstructed using 24 iterations with $45 \%$ smoothing, $100 \%$ resolution and summing all projections together to produce a 1-hour perfusion scan.

\subsection{SPECT Analysis}

All SPECT images were quantitatively analyzed using INVIA Corridor-4DM software (MI, USA) on reconstructed gated images. A contour was fitted around the right ventricular blood pool with the crosshairs located in the middle of the RV. The valve plane was oriented to be in line with the shortest line of the RV wall on the vertical long axis slice.

\subsection{Determination of Protein Concentration}

At time of sacrifice, hearts were excised and RV was separated from LV and septum. The dissected tissues were immediately frozen in liquid nitrogen and stored at $-80^{\circ} \mathrm{C}$ for later use. The frozen tissue samples were hand powdered with mortar and pestle under liquid nitrogen. Protein was combined with lysis buffer and homogenized using an electric homogenizer ( $2 \mathrm{x} 10 \mathrm{sec})$. TritonX-100 was added to tissue homogenate and kept on ice for 1 hour for cell 
lysis to occur. The samples were centrifuged for 15 mins. at $10,000 \mathrm{rpm}$ at $4^{\circ} \mathrm{C}$, supernatant was aspirated and frozen at $-80^{\circ} \mathrm{C}$ in $50 \mathrm{ul}$ aliquots. Protein concentration per sample was determined using a BCA Assay. In brief, 5 dilutions of Bovine Serum Albumin (BSA) protein standard were prepared in the following concentrations: $1000 \mathrm{ug} / \mathrm{ml}, 500 \mathrm{mg} / \mathrm{ml}, 250$ $\mathrm{mg} / \mathrm{ml}, 125 \mathrm{mg} / \mathrm{ml}, 62.5 \mathrm{mg} / \mathrm{ml}$ and blank, and pipette in $25 \mathrm{ul}$ volumes in triplicates into individual wells. Samples were prepared in a 1:30 dilution (sample:lysis buffer) and pipette in $25 \mathrm{ul}$ volumes in triplicates into individual wells. 200ul of BCA working reagent (50:1, BCA: 4\% Cupric Acid) was added to each well and the plate was incubated for 30 mins. at $30 \mathrm{rpm}$ at $40^{\circ} \mathrm{C}$. Samples were cooled to room temperature then read at an absorbance of 560 nm (Polar star Galaxy). A standard curve was generated of absorbance vs. total protein concentration and protein concentrations of original samples were determined.

\subsection{Western Blot Analysis}

Samples were boiled for 5 minutes at $95-100^{\circ} \mathrm{C}$ and centrifuged at $10000 \mathrm{rpm}$. Tissue lysate (20 ug of protein) was loaded into each well of an $8 \%$ sodium dodecyl sulfatepolyacrylamide gel and run at a voltage set to $160 \mathrm{mV}$ for approximately 1.5 hours. The proteins were then transferred onto an Immobolin-PVDF membrane overnight at $4^{\circ} \mathrm{C}$ under 40V, $0.12 \mathrm{~A}$ and $40 \mathrm{~W}$ settings. The membrane was blocked with 5\% non-fat milk in TBST for 1 hour under constant agitation and then washed several times with TBST before being incubated overnight at $4^{\circ} \mathrm{C}$ with constant agitation with primary antibody: rabbit anti-GLUT4 (1:1000 with $2.5 \%$ non-fat milk in TBST) and mouse anti-GAPDH (1:4000 with $2.5 \%$ nonfat milk in TBST). Membrane was washed several times with TBST to remove residual primary antibody then incubated for 1 hour at room temperature with the respective 
secondary antibody: goat anti-rabbit (1:5000 with 2.5\% non-fat milk in TBST) and donkey anti-mouse (1:2000 with 2.5\% non-fat milk in TBST). Membranes were prepped for protein visualization using enhanced chemilluminescence substrate for Western Blotting (Perkin Elmer Health Sciences, Toronto, Canada) and imaged using FlourChem 9900 Imaging System (AlphaInnotech/Cell Biosciences, CA, USA). Digital images were quantitively analyzed using AlphaEase FC software with band densities being normalized to GAPDH and expressed as percentage.

\subsection{Statistics}

Values were expressed as mean $+/$ - standard deviation. Data were compared using student's t-test or one-way analysis of variance (ANOVA) with multiple comparisons carried out between groups using Bonferroni's post hoc comparison. Pearson correlation and simple regression were used to relate RV FDG SUV and hemodynamics as well as RV function and a p-value $<0.05$ was considered significant. Statistical testing was done on STATA version 10. 


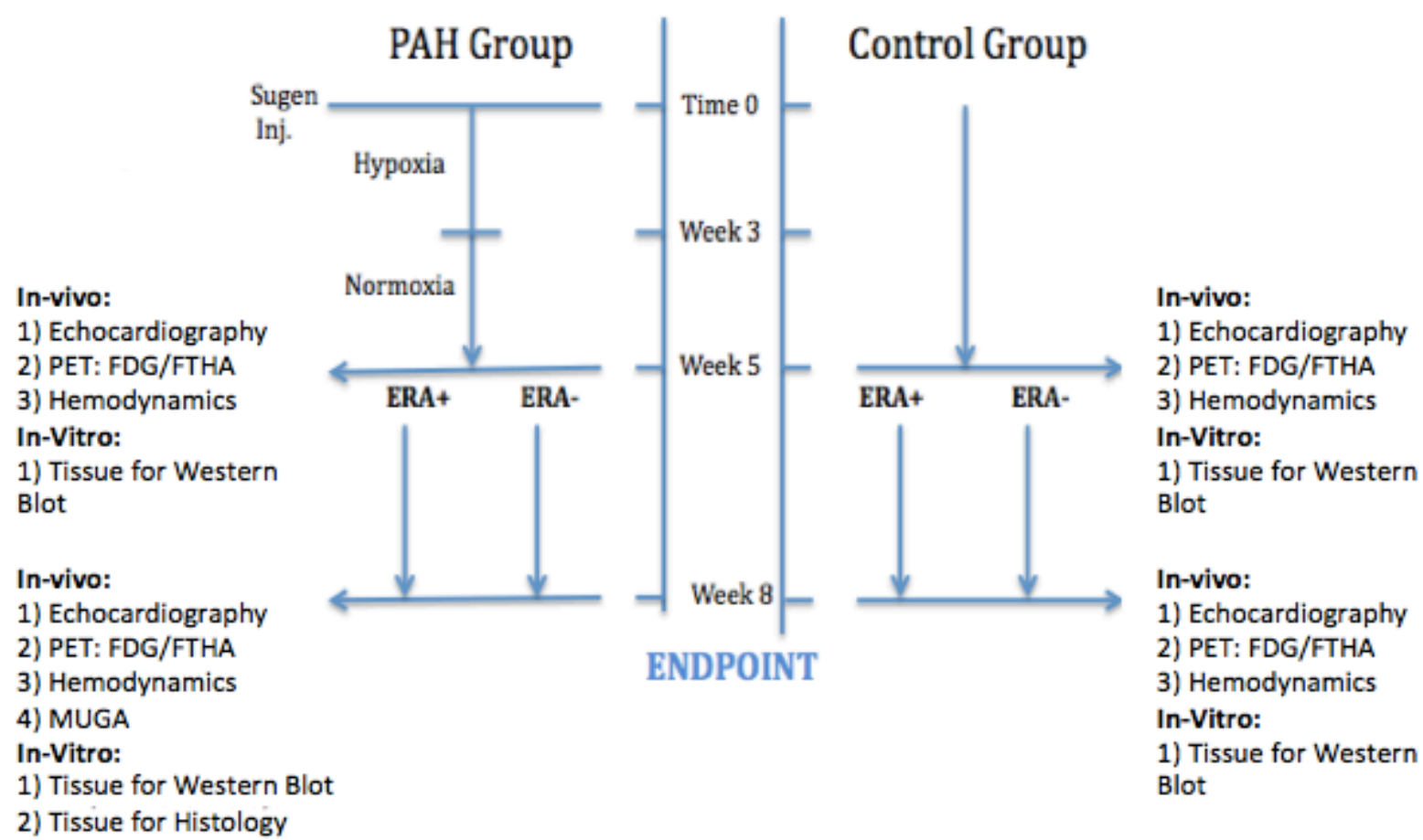

Figure 3.1. Experimental Protocol for the evaluation of Endothelin Receptor Antagonist Macitentan, on right ventricular and pulmonary artery substrate utilization in SuHx rodent model of severe PAH. In-vivo and in-vitro data was collected 5 and 8weeks post Sugen injection. 


\subsection{RESULTS}

\subsection{Development and Progression of PAH}

The SuHx model resulted in the development of severe and progressive PAH. Doppler measurements of PAAT were significantly reduced in comparison to controls at week 5 SuHx $(30.3 \pm 2.2 \mathrm{~ms}$ control, $20.6 \pm 3.7 \mathrm{~ms}$ week $5 \mathrm{SuHx}, \mathrm{p}<0.01$ compared to control $)$ and week $8 \mathrm{SuHx}(17.3 \pm 2.3 \mathrm{~ms}, \mathrm{p}<0.05$ compared to control). (Figure 4.1) By week $8 \mathrm{SuHx}$ the animals had severe PAH with the development of a characteristic mid-systolic notch on the PAAT signal. (Figure 4.1) Invasive measurements of right ventricular systolic pressure demonstrated a progressive trend with increases in pressure over 8 weeks $(27.1 \pm 1.0 \mathrm{mmHg}$ control, $53.6 \pm 9.2 \mathrm{mmHg}$ at week $5 \mathrm{SuHx}$ and $60.6 \pm 21.5 \mathrm{mmHg}$ at week $8 \mathrm{SuHx}, \mathrm{p}>0.05$ ). (Figure 4.2) This corresponded with a significant increase in RV fractional weight in week 8 SuHx rats compared to controls $(0.25 \pm 0.03 \mathrm{~g}$ control vs. $0.47 \pm 0.1 \mathrm{~g}$ at week $8 \mathrm{SuHx}$, $\mathrm{p}<0.01$ ). (Figure 4.3)

\subsection{Effects of Macitentan on PAH Severity}

Three weeks of treatment with $30 \mathrm{mg} / \mathrm{kg} /$ day of Macitentan showed a significant reduction in PAH severity compared to rats that were not given treatment. This was demonstrated by an increase in PAAT measured in week $8 \mathrm{SuHx}+$ ERA rats $(17.3 \pm 2.3 \mathrm{~ms}$ in untreated vs. 24.4 $\pm 3.9 \mathrm{~ms}$ in treated animals, $\mathrm{p}<0.001$ ). (Figure 4.4) There was a corresponding decrease in invasive measurements of RVSP, but did not reach statistical significance $(60.6 \pm 21.5$ $\mathrm{mmHg}$ in untreated vs. $46.9 \pm 10.7 \mathrm{mmHg}$ in treated animals, $\mathrm{p}>0.05$ ). (Figure 4.5) 
RV function was measured by SPECT imaging using Tc-99m blood flow tracer. Macitentan resulted in a significant increase in RV ejection fraction at week $8(53 \pm 9.9 \%$ in untreated vs. $73 \pm 4.8 \%$ in treated animals, $\mathrm{p}<0.01$ ). (Figure 4.6)

Pulmonary artery hypertension induced by the SuHx model demonstrated the expected histopathologic changes in the pulmonary arteriole (Pietra et al., 1989). (Figure 4.7) This consisted of hematoxylin and eosin staining reflecting medial hypertrophy with concentric layers of myofibroblasts, collagen and elastic fibers. Masson's trichrome stain was used to display the dense collagen fibres and smooth muscle actin stain identified evidence of smooth muscle cell hypertrophy in the vessel walls. Rats that received Macitentan treatment displayed a significantly lesser grade of histopathologic changes in small pulmonary arteries compared to the untreated group that displayed observable vascular occlusions. In addition, untreated rats had significant RV hypertrophy and remodeling which was observed to a lesser degree in rats with Macitentan treatment. (Figure 4.8)

\subsection{Cardiac Metabolic Adaptation to PAH}

\subsubsection{FDG Uptake}

There was a significant increase in measured RV FDG uptake at week $5 \mathrm{SuHx}$ compared to controls (SUV $4.06 \pm 1.9$ vs. $1.56 \pm 0.4, \mathrm{p}<0.01$ ). However there was no further significant increase in RV FDG uptake from week $5 \mathrm{SuHx}$ to week $8 \mathrm{SuHx}$ (SUV $4.06 \pm 1.9$ vs. $4.0 \pm$ 1.6, $\mathrm{p}>0.05$ ) Macitentan resulted in a significant decrease in RV FDG uptake at week 8 (SUV $4.0 \pm 1.6$ in untreated vs. $2.5 \pm 0.9$ in treated animals, $p<0.01$ ). (Figures 4.9A, 4.9B, 4.9C and 4.9D) Kinetic analysis of rMGU showed similar results with significantly increased myocardial glucose utilization with the onset of PAH at week $5 \mathrm{SuHx}$ compared to control (rMGU $0.52 \pm 0.2$ vs. $0.20 \pm 0.1, \mathrm{p}<0.01$ ). (Figure 4.10) Protein expression of GLUT 
4 was significantly increased with the onset of PAH and reduced following Macitentan treatment (Figures 4.11A and 4.11B), validating imaging findings. RV FDG uptake at week $8 \mathrm{SuHx}$ was significantly correlated with invasive pulmonary artery systolic pressure measurements at the same timepoint $(\mathrm{r}=0.87, \mathrm{p}=0.001)$ (Figure 4.12) and a negative correlation was demonstrated between RV FDG uptake and RV ejection fraction $(\mathrm{r}=-0.72$, $\mathrm{p}=0.01$ ). (Figure 4.13) In contrast, there was no significant change in LV FDG uptake throughout the period of study (SUV $3.8 \pm 1.2$ in controls, $4.8 \pm 2.1$ at week $5 \mathrm{SuHx}, 4.3 \pm$ 2.0 at week $8 \mathrm{SuHx}, 3.7 \pm 1.7$ at week $8 \mathrm{SuHx}+\mathrm{ERA}, \mathrm{p}>0.05$ for all comparisons). (Figure 4.14).

\subsubsection{FTHA Uptake}

There was a non-significant trend towards increased RV FTHA uptake at week $5 \mathrm{SuHx}$ compared to controls (SUV $3.1 \pm 1.1$ vs. $1.5 \pm 0.4, \mathrm{p}>0.05$ ), and similarly, a non-significant trend for RV FTHA uptake to decrease at week 8 SuHx (SUV $3.1 \pm 1.1$ at week 5 SuHx vs. $2.5 \pm 1.5$ at week $8 \mathrm{SuHx}, \mathrm{p}>0.05$ ). (Figures $4.15 \mathrm{~A}$ and 4.15C) There were no further significant changes in RV FTHA uptake following treatment with Macitentan. (SUV $2.5 \pm$ 1.5 in untreated vs. $2.7 \pm 0.7$ in treated animals, $p>0.05$ ). (Figures 4.15B and 4.15D) There was no significant change in LV FTHA uptake throughout the period of study. (SUV $3.2 \pm$ 0.6 in controls, $3.8 \pm 1.7$ at week $5 \mathrm{SuHx}, 3.8 \pm 1.8$ at week $8 \mathrm{SuHx}, 3.8 \pm 0.9$ at week 8 $\mathrm{SuHx}+\mathrm{ERA} \mathrm{p}>0.05$ for all comparisons). (Figure 4.16)

\subsection{Lung Metabolic Adaptation to PAH}

PET scans were evaluated to identify any changes in substrate uptake in the lung parenchyma associated with the SuHx model. This study did not demonstrate any significant changes in lung FDG uptake between controls and week 8 in treated or untreated animals. 
(SUV $0.49 \pm 0.1$ in controls, $0.46 \pm 0.2$ at week $5 \mathrm{SuHx}, 0.40 \pm 0.2$ at week $8 \mathrm{SuHx}, 0.39 \pm$ 0.1 at week $8 \mathrm{SuHx}+$ ERA, $\mathrm{p}>0.05$ for all comparisons). (Figure 4.17) In addition there were no significant changes in lung FTHA uptake over the same time period (SUV $0.31 \pm$ 0.1 in controls, $0.35 \pm 0.1$ week $5 \mathrm{SuHx}, 0.35 \pm 0.2$ week $8 \mathrm{SuHx}, 0.30 \pm 0.1$ at week $8 \mathrm{SuHx}$ + ERA, p $>0.05$ for all comparisons). (Figure 4.18). 

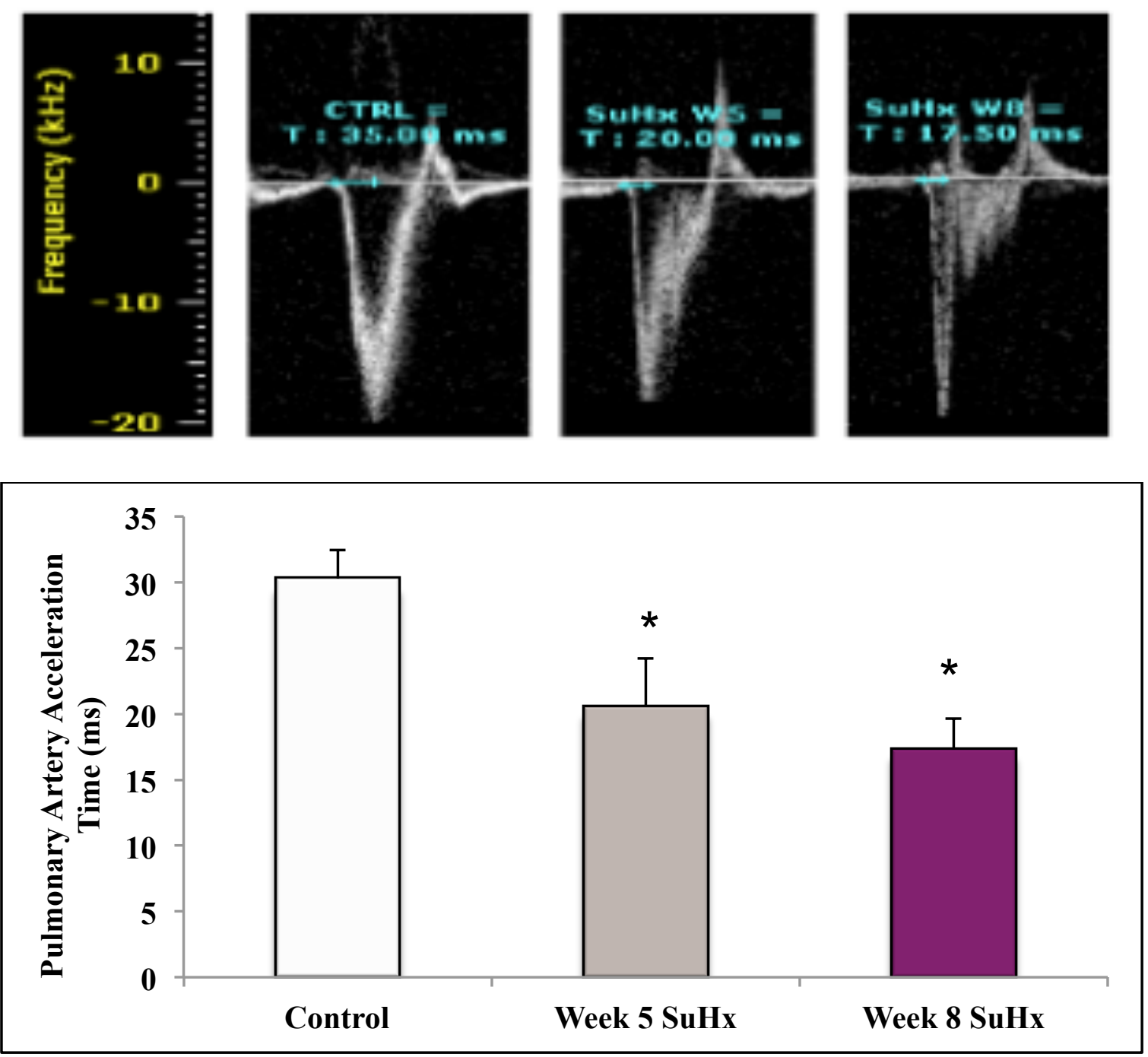

Figure 4.1: Development of PAH demonstrated by echocardiography. A) Representative images of pulsed-wave Doppler analysis B) Pulsed-wave Doppler measurements of PAAT. Control n=4, Week 5 SuHx $n=10$, Week 8 SuHx $n=10$. * $p<0.001$ compared to control, oneway ANOVA, Bonferroni post hoc comparison. 


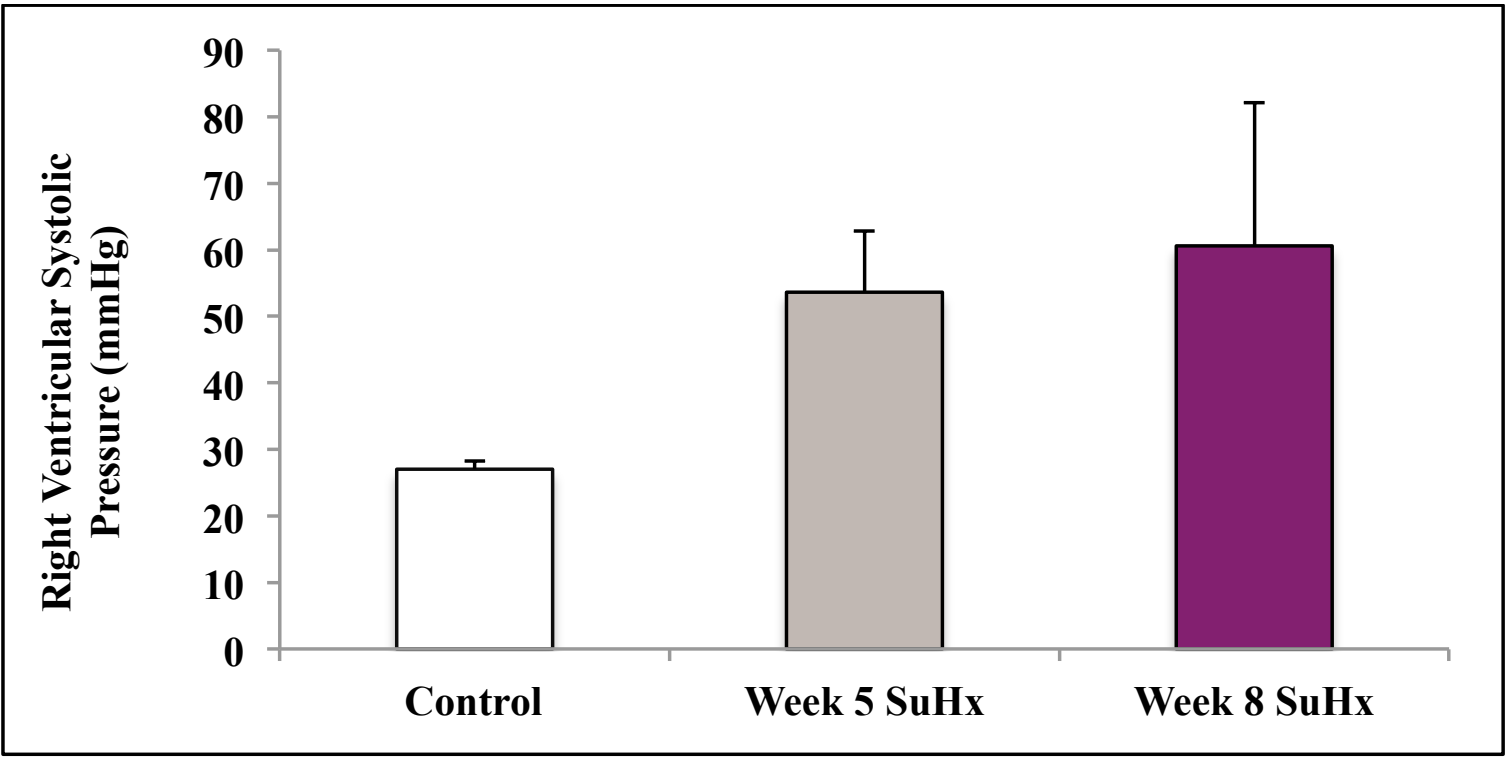

Figure 4.2: Hemodynamic effect of SuHx exposed PAH rats. Invasive catheterization data showing measurements of RVSP. Control n=3, Week 5 SuHx n=2, Week 8 SuHx n=6. $\mathrm{p}>0.05$, One-way ANOVA, Bonferroni post hoc comparison.

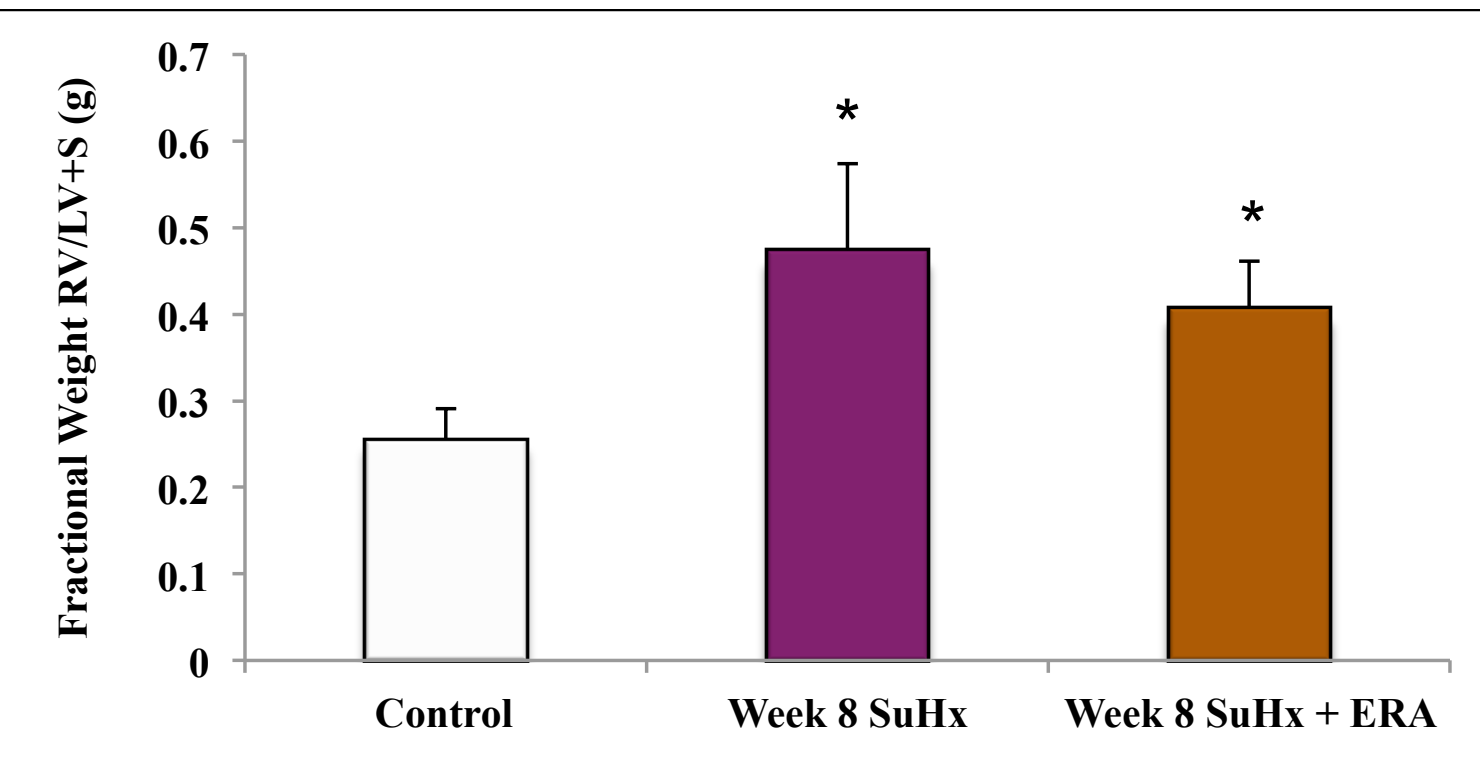

Figure 4.3: Measure of right ventricular hypertrophy following Macitentan treatment.

Fractional weight ratio $(\mathrm{RV} / \mathrm{LV}+\mathrm{S})$. Control $\mathrm{n}=6$, Week 8 SuHx $\mathrm{n}=6$, Week 8 SuHx + ERA $\mathrm{n}=3 .{ }^{*} \mathrm{p}<0.01$ compared to control, one-way ANOVA, Bonferroni post hoc comparison. 


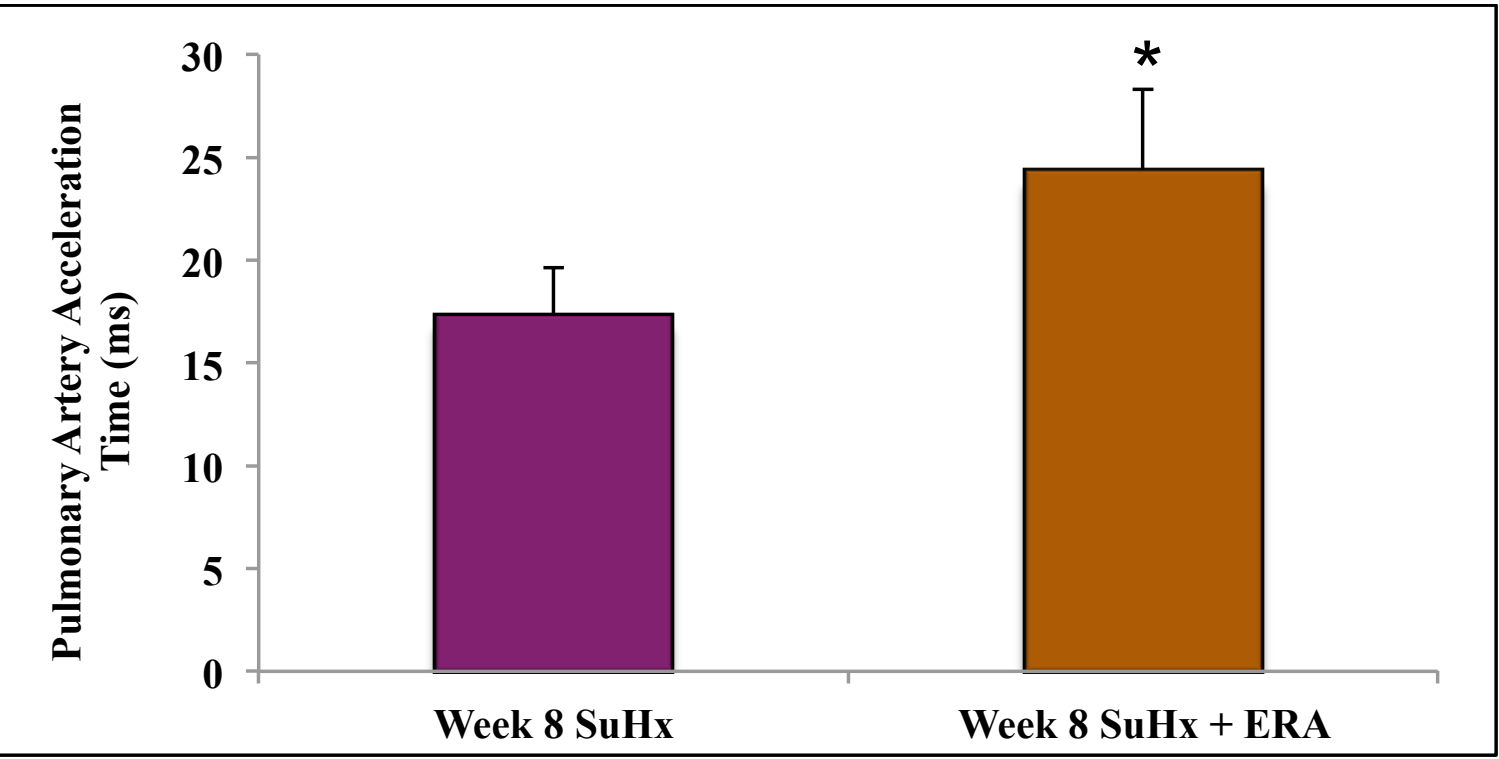

Figure 4.4: Effect of Macitentan treatment on PAAT measurements. Pulsed-wave Doppler measurements of PAAT. Week 8 SuHx $n=10$, Week 8 SuHx + ERA n=11. $* \mathrm{p}<0.001$, Student's t-test.

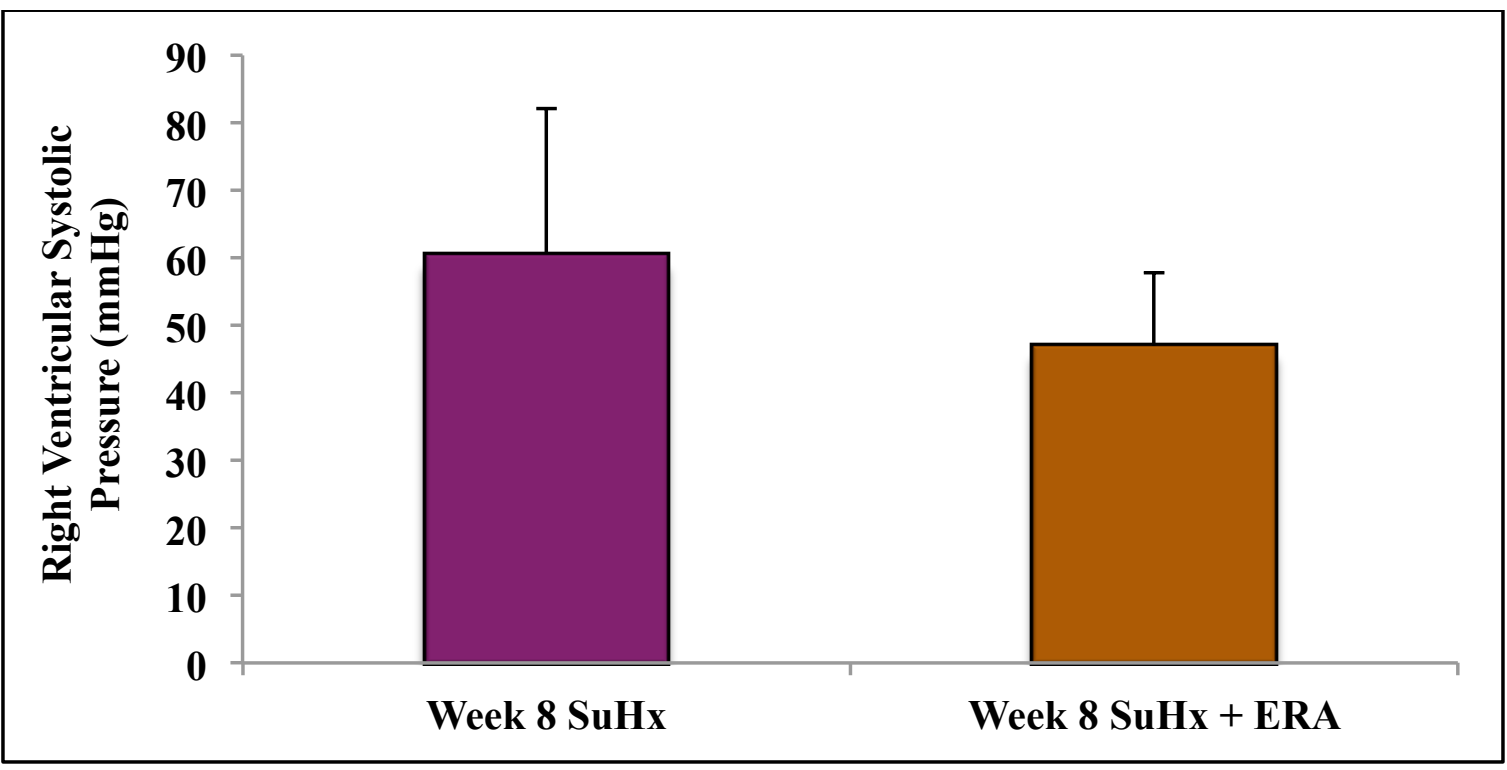

Figure 4.5: Hemodynamic effect of Macitentan treatment on SuHx exposed PAH rats. Invasive catheterization data showing measurements of RVSP. Week 8 SuHx n=6, Week 8 SuHx + ERA n=5. p >0.05, Student's t-test. 


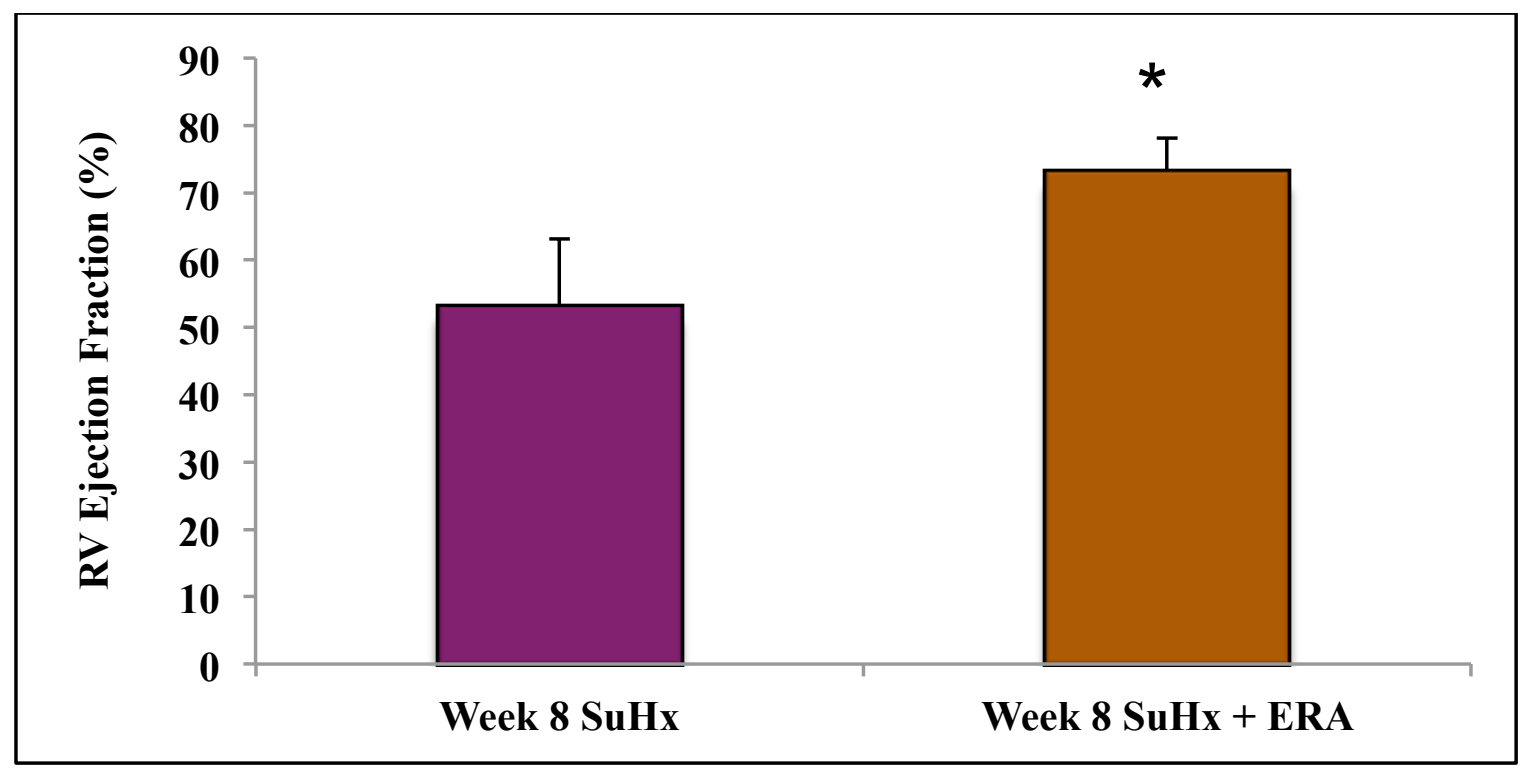

Figure 4.6: Effect of Macitentan on RV ejection fraction. RV function as assessed by SPECT imaging using Tc-99m blood flow tracer. Week 8 SuHx n=5, Week 8 SuHx+ERA $\mathrm{n}=6 .{ }^{*} \mathrm{p}<0.01$, Student's t-test. 


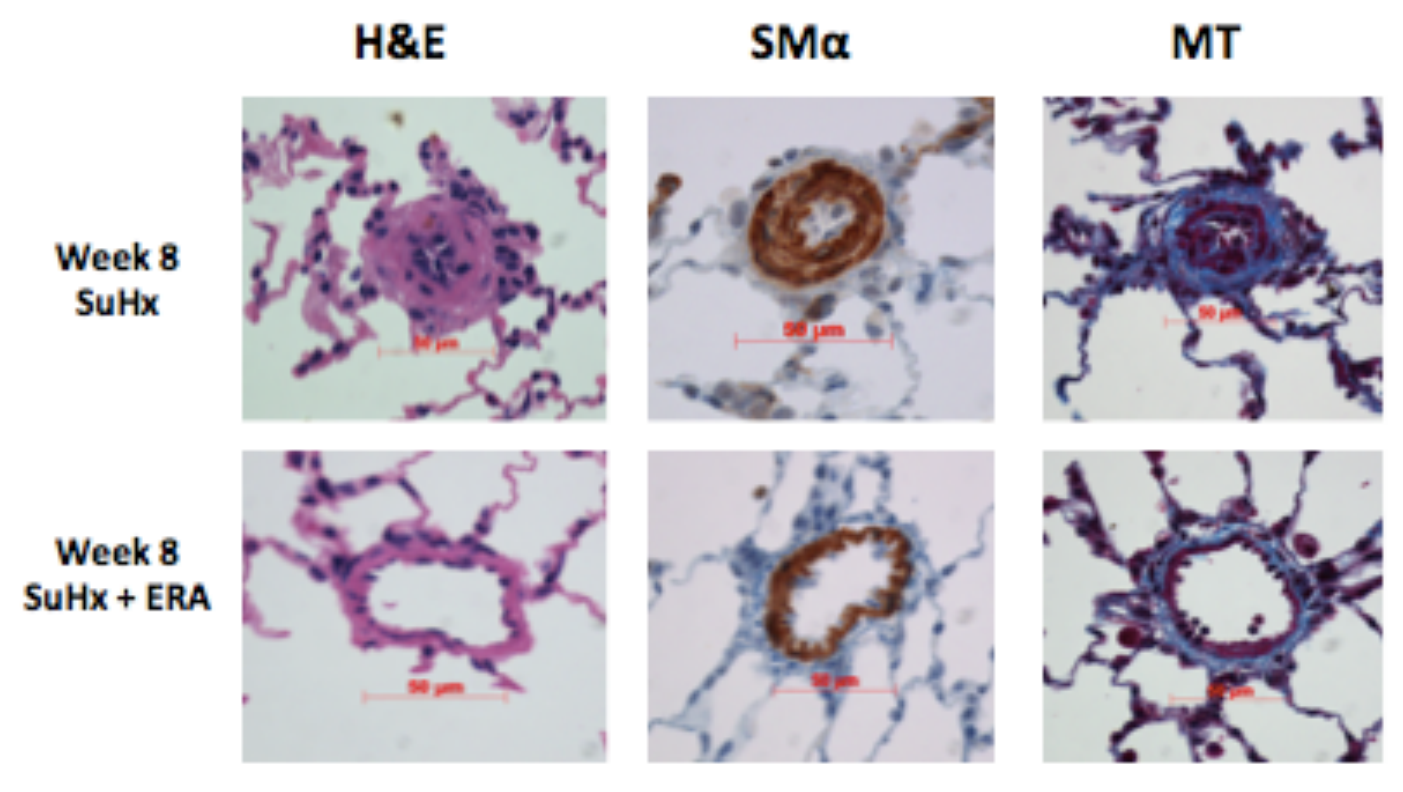

Figure 4.7: Representative histological and immunohistochemical images of small pulmonary arteries at week $8 \mathrm{SuHx}$ and following Macitentan treatment. Hematoxylin \& Eosin (H\&E) showing regression of medial thickening, smooth muscle actin (SM $\alpha$ ) displays reduced muscularization and Masson's Trichrome (MT) staining depicting fibrosis.
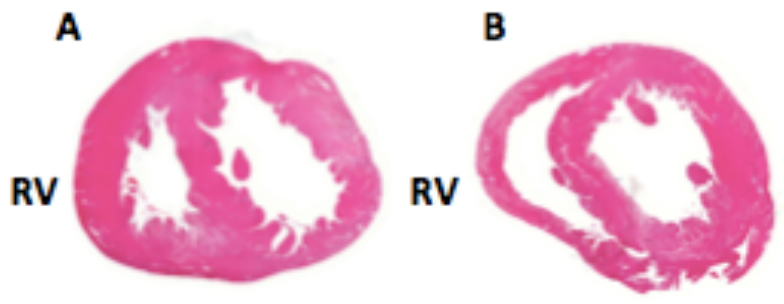

Figure 4.8: Effect of Macitentan treatment on RV hypertrophy. Representative cross section of the myocardium stained with hematoxylin \& eosin in A) untreated and B) Macitentan treated animals. 
A

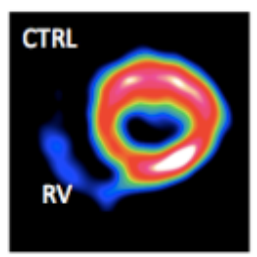

C
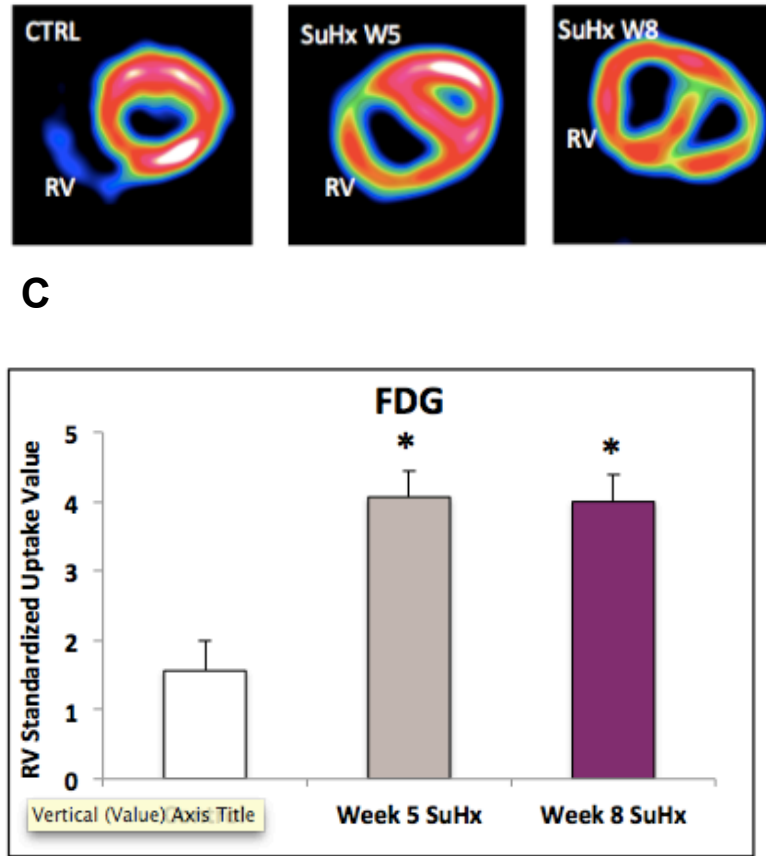

B
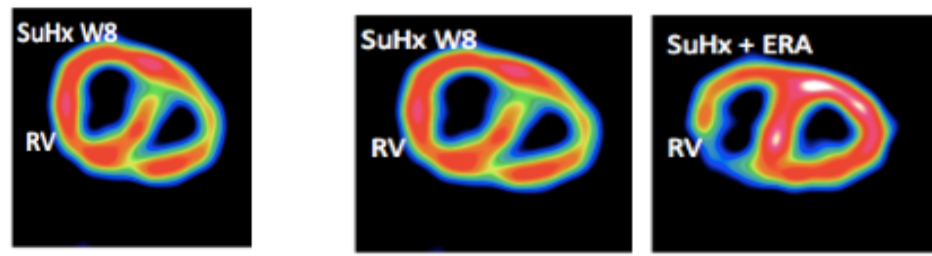

D

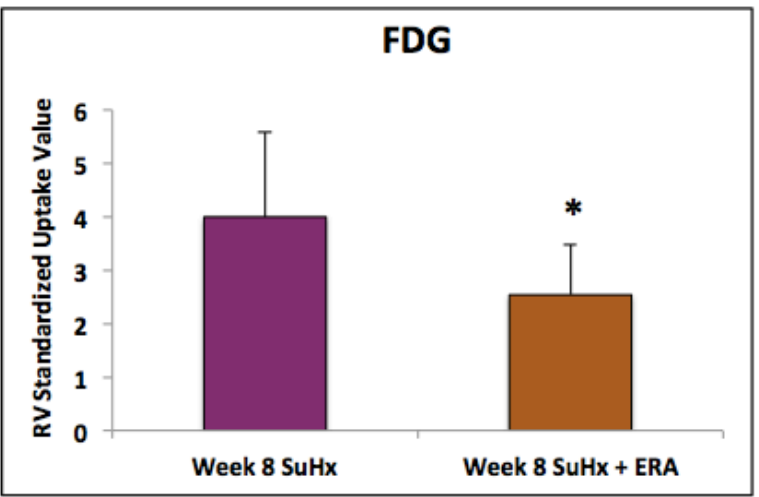

Figure 4.9: Quantification of RV FDG uptake measured by PET. A) Representative short axis FDG PET images throughout the development of PAH B) Representative short axis FDG PET images following Macitentan treatment C) Sequence of metabolic changes throughout the development of PAH. Control n=4, Week 5 SuHx n=9, Week 8 SuHx. ${ }^{*} \mathrm{p}<0.05$ compared to control, one-way ANOVA, Bonferroni post hoc comparison. D) FDG uptake following Macitentan treatment. Week 8 SuHx n=8, Week 8 SuHx + ERA n=8. $* \mathrm{p}<0.05$, Student's t-test. 


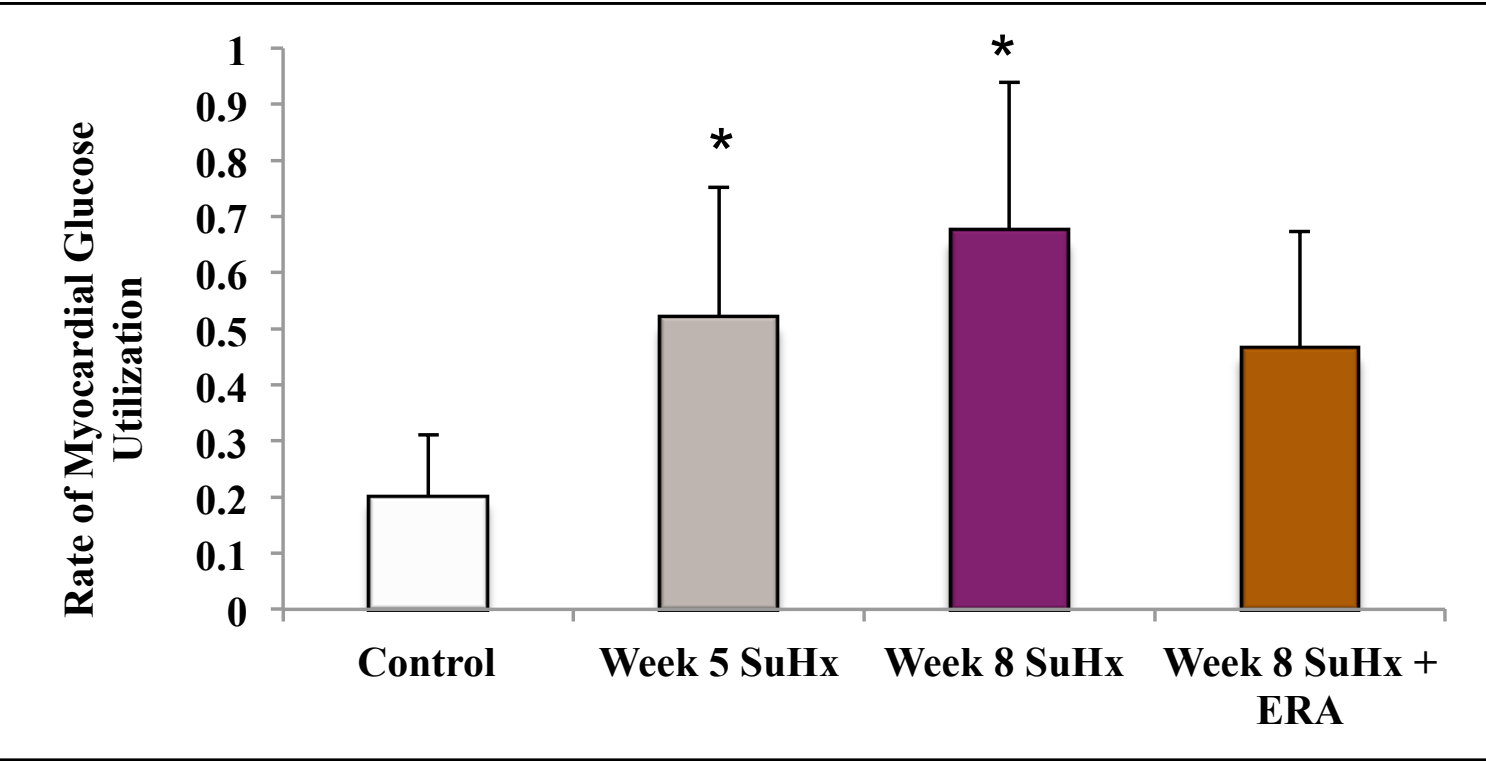

Figure 4.10: Kinetic Analysis of Rate of Myocardial Glucose Utilization measured in

the RV. Net influx of FDG tracer into RV tissue. Control $n=4$, Week $5 \mathrm{SuHx} n=9$, Week 8 SuHx $n=8$, Week 8 SuHx + ERA $n=8 . * p<0.01$ compared to control, one-way ANOVA, Bonferroni post hoc comparison. 


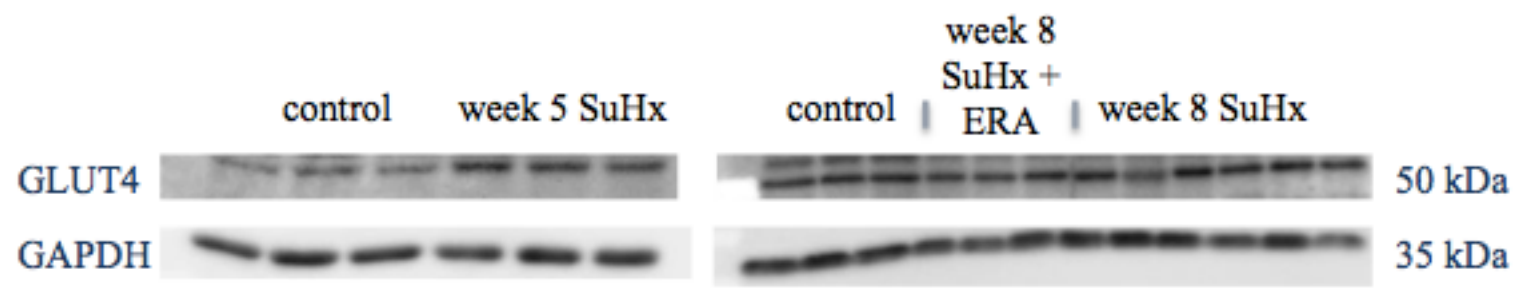

A

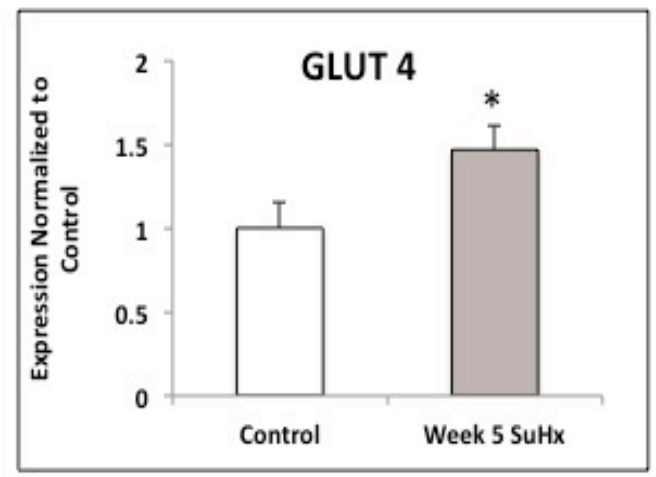

B

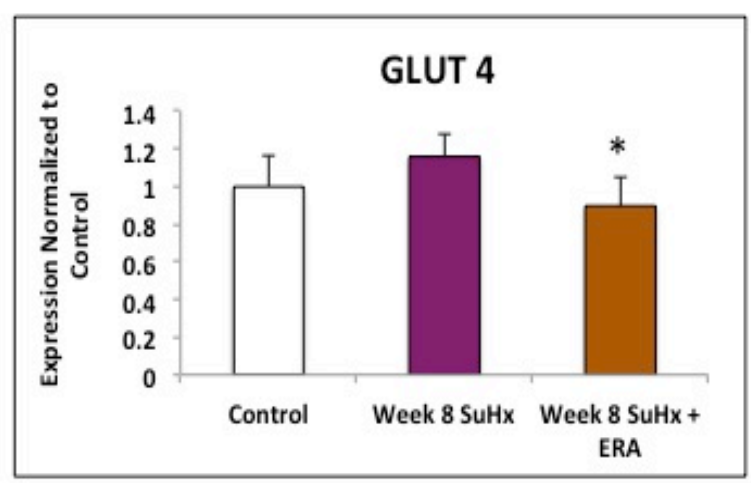

Figure 4.11: Western blot analysis of GLUT4 in RV tissue normalized to GAPDH control. A) Relative expression at week 5. Control $n=3$, Week $5 \mathrm{SuHx} n=3$. ${ }^{*} \mathrm{p}<0.05$, Student's t-test. B) Relative expression at week 8 . Control $n=3$, Week 8 SuHx n=6, Week 8 SuHx + ERA $n=3 .{ }^{*} \mathrm{p}<0.05$ compared to control, one-way ANOVA, Bonferroni post hoc comparison 


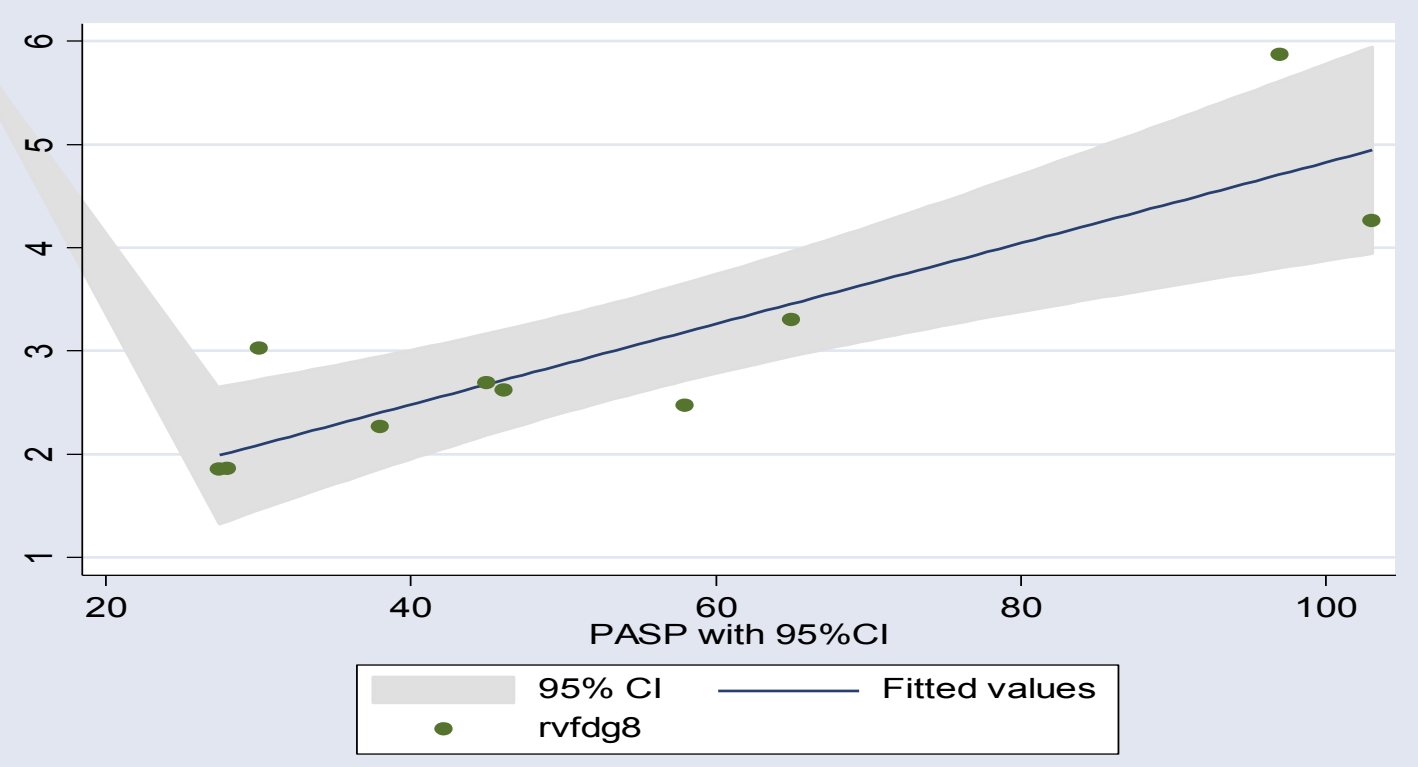

Figure 4.12: Pearson correlation between RV FDG SUV and pulmonary artery systolic pressure (PASP). A strong positive association between RV FDG uptake and increased hemodynamics $(r=0.8709, \mathrm{p}=0.001)$.

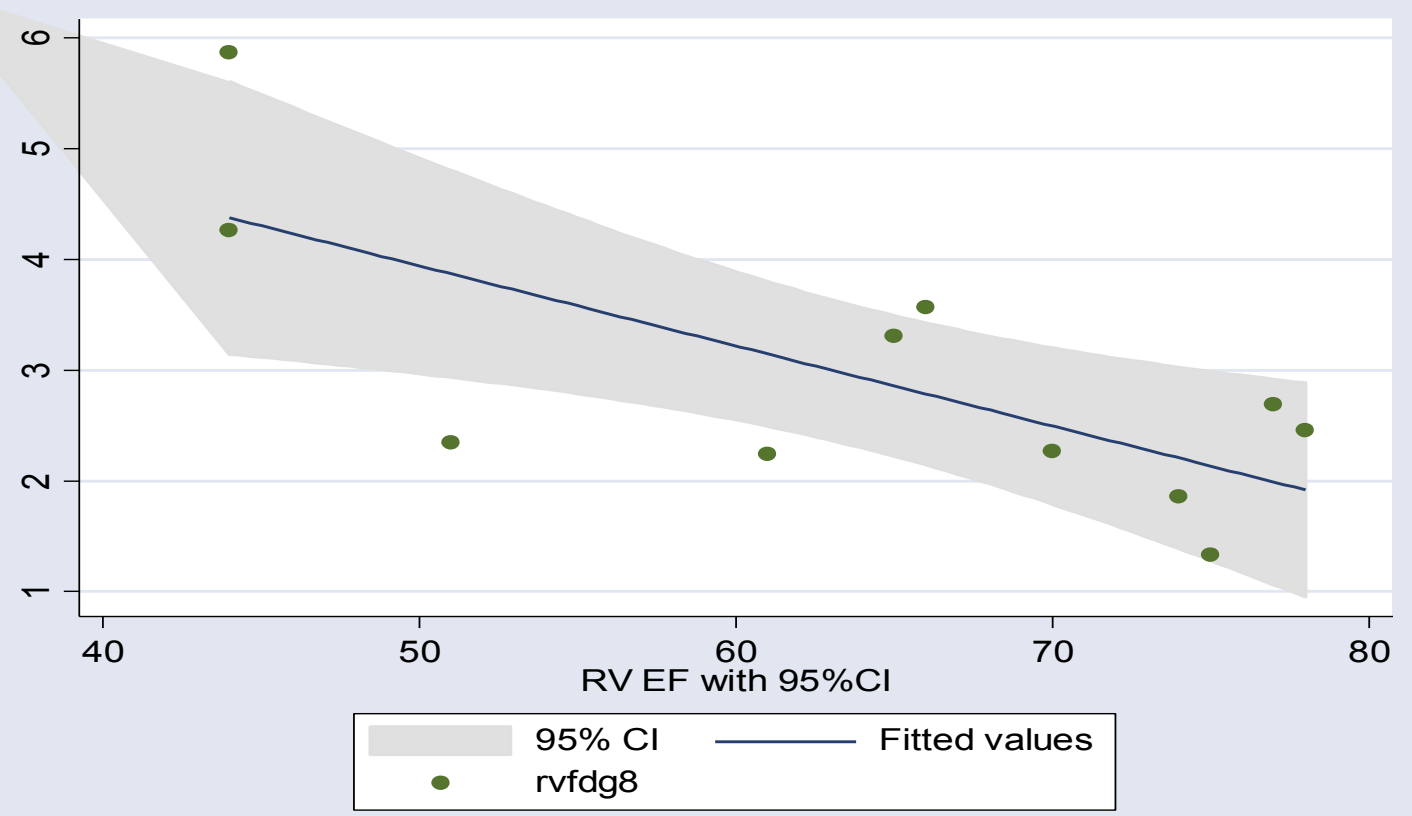

Figure 4.13: Pearson correlation between RV FDG SUV and RVEF. A strong inverse relationship between RV FDG uptake and RV function $(r=-0.719, p=0.01)$. 


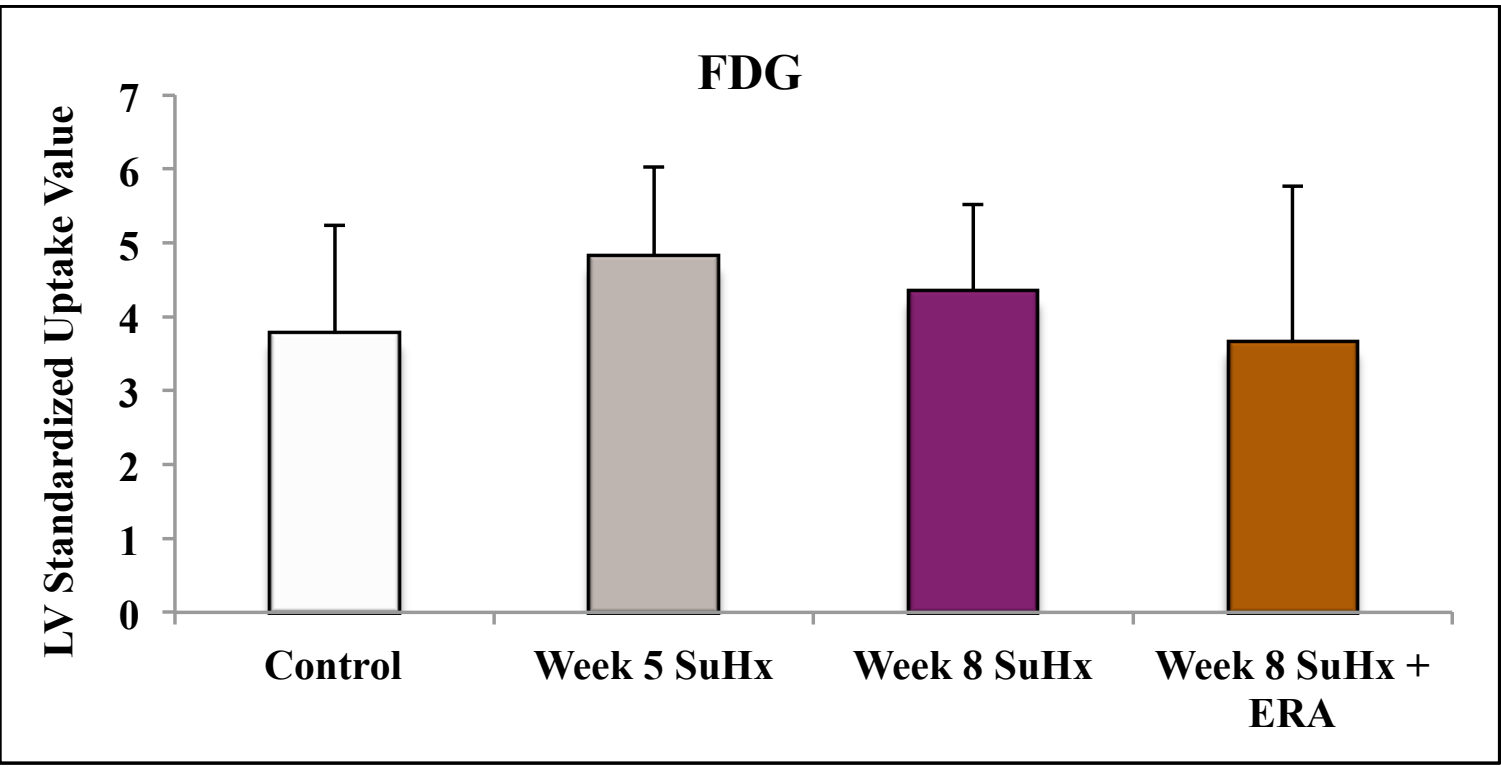

Figure 4.14: Quantification of LV FDG uptake measured by PET. Control n=4, Week 5 SuHx n=9, Week 8 SuHx n=8, Week 8 SuHx + ERA n=8. p>0.05, One-way ANOVA, Bonferroni post hoc comparison. 
A

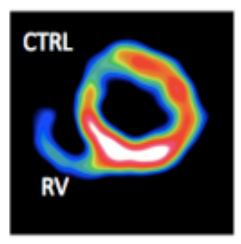

C
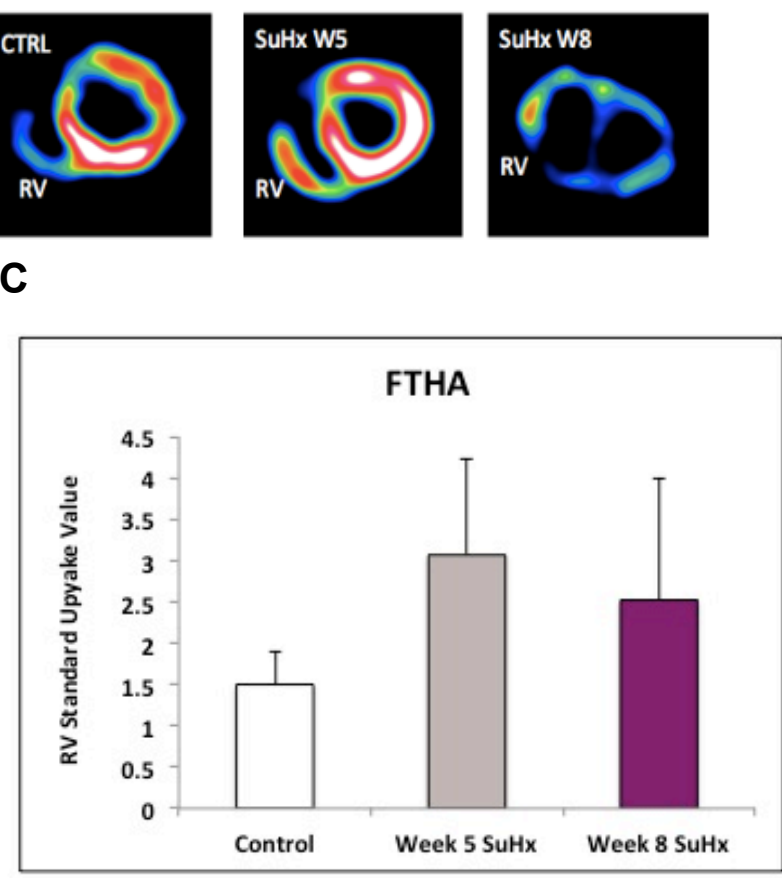

B

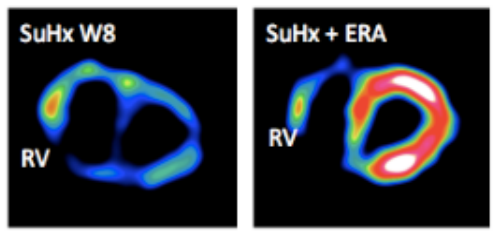

D

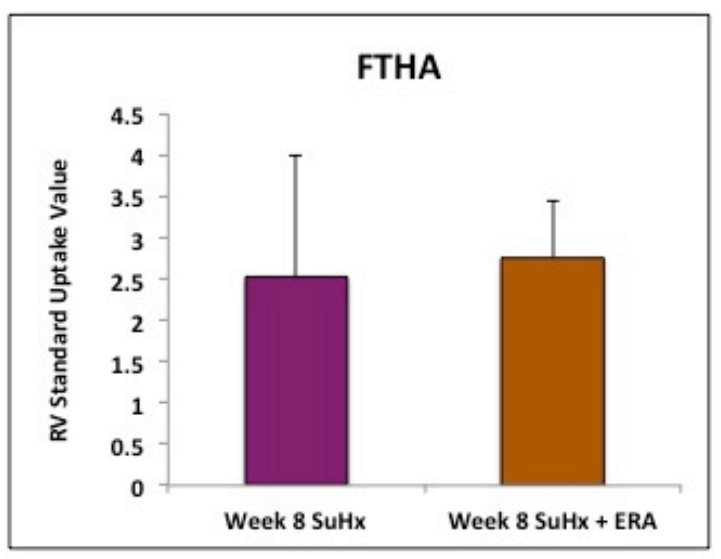

Figure 4.15: Quantification of RV FTHA uptake measured by PET. A) Representative short axis FTHA PET images throughout the development of PAH B) Representative short axis FTHA PET images following Macitentan treatment C) Sequence of metabolic changes throughout the development of PAH. Control n=4, Week 5 SuHx n=5, Week 8 SuHx n=7. $\mathrm{p}>0.05$, One-way ANOVA, Bonferroni post hoc comparison.D) FTHA uptake following Macitentan treatment. Week 8 SuHx n=7, Week 8 SuHx + ERA n=7. p>0.05, Student's ttest. 


\section{FTHA}

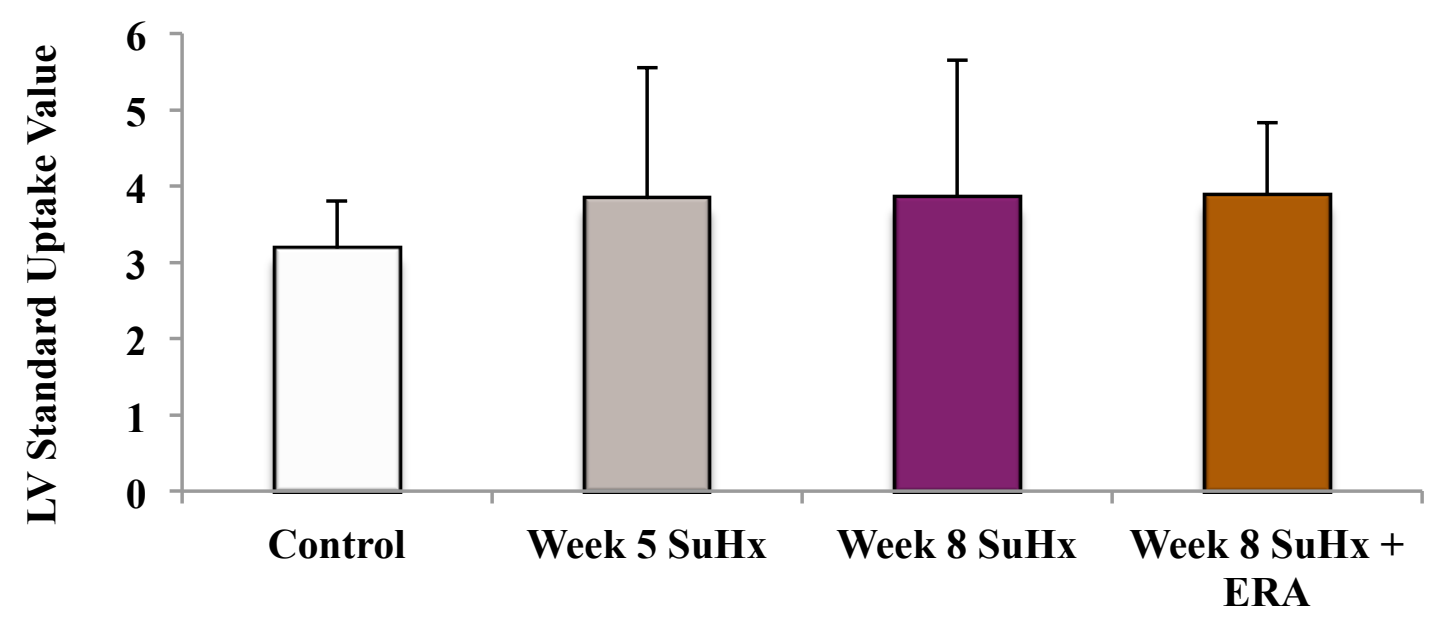

Figure 4.16: Quantification of LV FTHA uptake measured by PET. Control n=4, Week

5 SuHx n=5, Week 8 SuHx n=7, Week 8 SuHx + ERA $n=7 . p>0.05$, One-way ANOVA, Bonferroni post hoc comparison. 


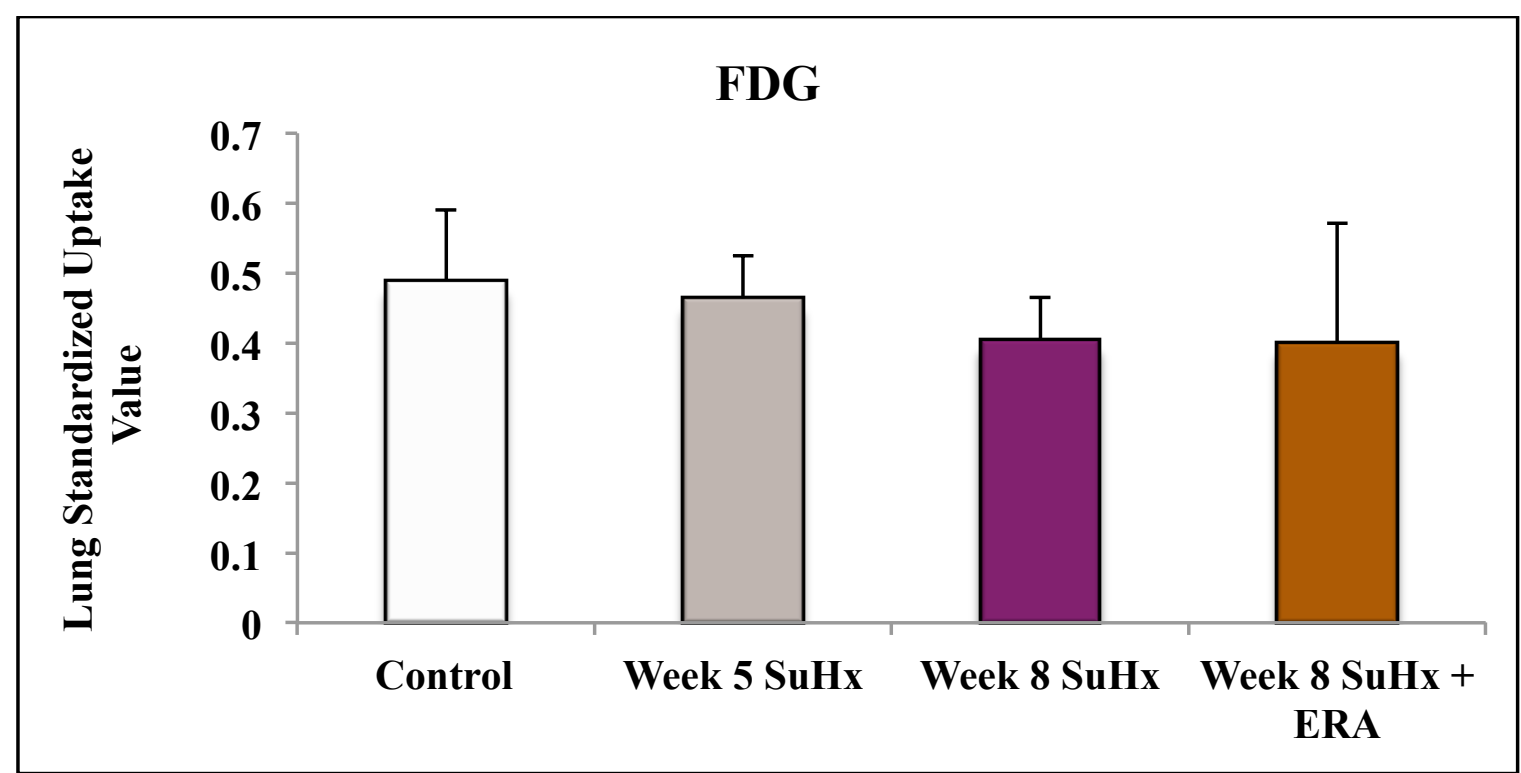

Figure 4.17: Quantification of lung FDG uptake measured by PET. Control $n=4$, Week 5 SuHx n=9, Week 8 SuHx n=8, Week 8 SuHx + ERA $n=8 \cdot p>0.05$, One-way ANOVA, Bonferroni post hoc comparison. 


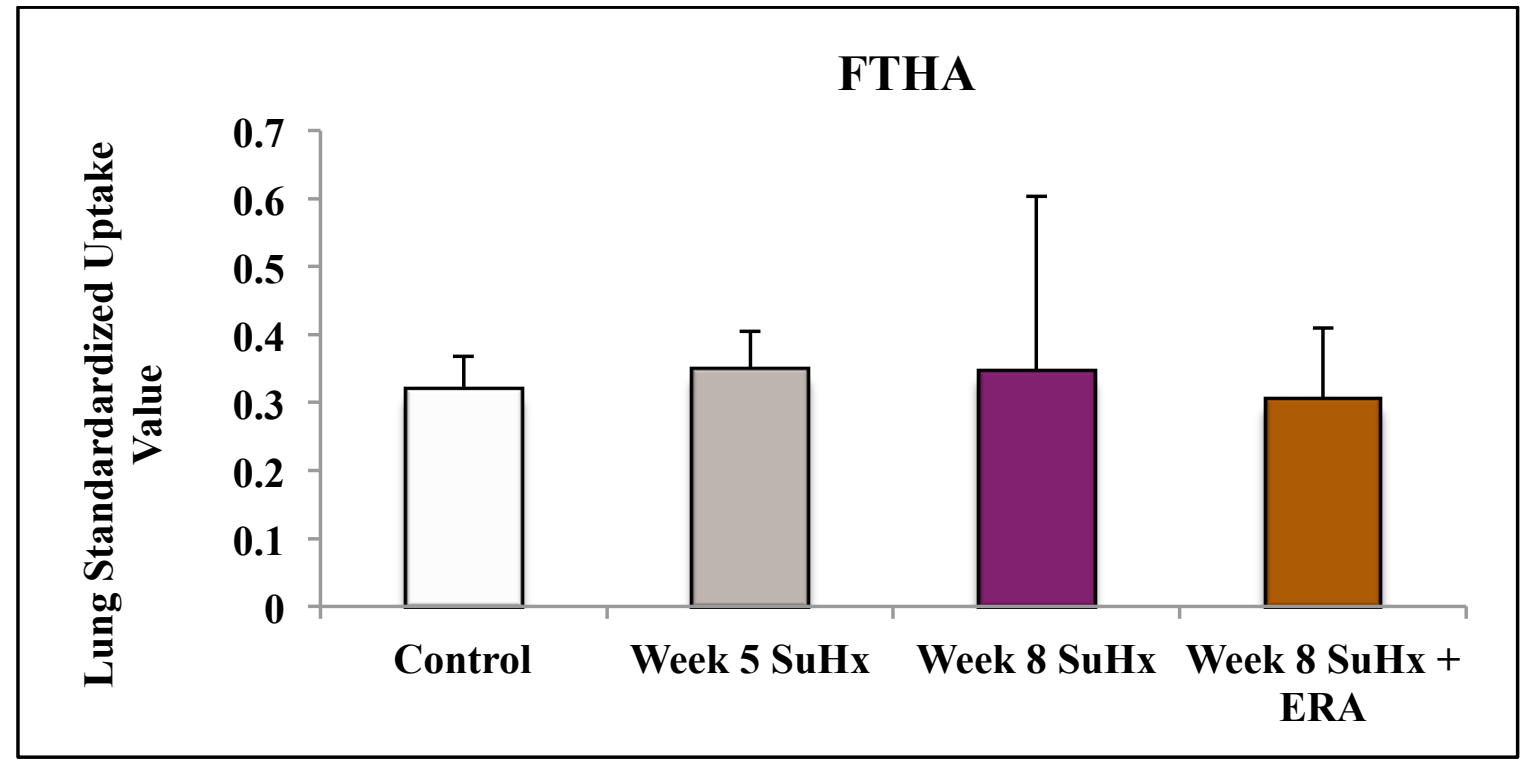

Figure 4.18: Quantification of lung FDG uptake measured by PET. Control n=4, Week 5

SuHx n=5, Week 8 SuHx n=7, Week 8 SuHx + ERA n=7. p>0.05, One-way ANOVA, Bonferroni post hoc comparison. 


\subsection{DISCUSSION}

In this study we used serial non-invasive PET imaging with FDG and FTHA to evaluate glucose and fatty acid metabolic changes throughout the progression of $\mathrm{PAH}$ in a SuHx rat model and correlated these changes with RV function and hemodynamics. This study represents the first use of FTHA PET to measure myocardial and lung fatty acid uptake over time and to evaluate the metabolic response to Macitentan, a new generation ERA recently approved by Health Canada for clinical use. The major novel findings of our study are as follows 1) The onset of PAH is associated with a metabolic shift characterized by increased fatty acid and glucose uptake in the RV; 2) Chronic treatment with Macitentan reduces FDG uptake in the RV, supported by a reduction in GLUT4; 3) There is a strong inverse correlation between reduction of RV FDG uptake and improvement in RV function following Macitentan treatment 4) There is a strong correlation between reduction of RV FDG uptake and improvement in hemodynamics following Macitentan treatment also depicted by regression in pulmonary vascular remodeling. Our study provides evidence that there is a metabolic switch described not only by changes in glucose metabolism but also FAO, and that these changes are associated with worsening of RV function and hemodynamics. Although the changes in glucose uptake were clear and consistent over time, there was greater inconsistency with respect to the changes in fatty acids. Obtaining a better overall understanding of changes in metabolic flux throughout progressive stages of PAH and the effects on RV function will offer the potential of developing more effective therapies and validate the use of PET imaging as a diagnostic tool or for monitoring response to treatment. 


\subsection{Development and Progression of PAH}

The SuHx model was chosen to study metabolic alterations and effect of ERA therapy in the setting of severe PAH. The model was associated with trending elevated RVSP measurements, which persisted from week $5 \mathrm{SuHx}$ to week $8 \mathrm{SuHx}$. This is consistent with other reports using this model (Taraseviciene-Stewart et al., 2001; Boogard et al., 2009; Abe et al., 2010; Kunita-Takanezawa et al., 2014). The developmental hemodynamic elevations exhibited in this model are also representative of clinical presentation of PAH (Steele et al., 2010). PAH is diagnosed by the presence of mPAP greater than $25 \mathrm{mmHg}$ in a patient's hemodynamic profile and is commonly the first test for evaluation of the disease (McGoon et al., 2009). SuHx proves to be a relevant model of severe PAH because of the achievement of high persisting pressures, which progress even upon re-exposure to normoxia (TarasevicieneSewart et al., 2001). In some rodent models such as chronic hypoxia or MCT, vascular remodeling may be reversed after rodents are re-exposed to normoxia, or with the passing of time post drug administration (Taraseviciene-Sewart et al., 2001; Sebkhi et al., 2003; Schermuly et al., 2004; FosoClozel et al., 2006). Inadequate animal models of PAH could be the reason for the disappointing clinical data. The notion that "almost everything inhibits monocrotaline-induced pulmonary hypertension" suggests that investigators should carry out pre-clinical research in additional animal models of PAH (Bauer et al., 2007).

To better characterize the severity of PAH in the animals, functional measurements were obtained at week $5 \mathrm{SuHx}$ and week $8 \mathrm{SuHx}$ using echocardiography. As expected, Doppler measurements showed progressive shortening in PAAT throughout the experimental time 
course as well as changes in pulmonary artery flow profiles corresponding to severity of PAH. In a healthy subject, pulmonary artery flow signal takes the form of a symmetrical round-shaped contour (Ginghina et al., 2009; Koskenvuo et al., 2010). As pulmonary artery pressures increase, RV stroke work increases, causing a triangular flow profile (Koskenvuo et al., 2010). Echocardiographic evaluation also revealed a characteristic mid-systolic notching in the pulmonary artery waveform at week $8 \mathrm{SuHx}$ time point caused by a pathologic wave reflection in the presence of excessive pressures.

Histological examination of the small pulmonary arteries (outer diameter $\sim 50 \mu \mathrm{m}$ ) at week 8 SuHx revealed significant vascular remodeling. Hematoxylin \& Eosin staining of the lung tissue showed intimal proliferation causing luminal occlusion. Medial changes were evaluated by immunostaining with smooth muscle cell marker $\alpha$-actin and revealed a pronounced degree of medial thickening. There was markedly more fibrosis surrounding the small pulmonary arteries. One advantage to using the SuHx model is the development of severe complex lesions, histologically indistinguishable from those found in humans. The role that plexiform lesions play in the development of PAH remains controversial and incompletely understood (White et al., 2007). Our model did not produce obvious observable plexiform lesions throughout the experimental time course. Previous studies have shown however, that a much longer duration of time is required for the development of complex lesions in the SuHx model. Abe and colleagues extensively described the formation of complex plexiform lesions in a SuHx model and found that it took 13-14 weeks from initial Sugen injection for these pathological lesions to appear (Abe et al., 2010). It is worth noting, that the development of complex plexiform lesions in humans is also delayed from onset of severe PAH and considered a late-stage change, consistent with Abe's observations in the 
rodent model (Heath et al., 1987; Abe et al., 2010). This evidence suggests that the progression of severe lesions is a product of chronic exposure to elevations in intravascular pressures rather than the source for hypertension (Abe et al., 2010). The progression of exuberant endothelial proliferation to complex plexiform lesion remains an area of investigation that warrants more study. The role that plexiform lesions play in the clinical worsening of PAH and response to therapy remains to be explored in more detail (Stenmark et al., 2009).

The temporal relationship between the histological and hemodynamic changes in the pulmonary vasculature were associated with the deterioration of RV function. The findings of this study underline the relevance and usefulness of the SuHx model to identify new mechanisms of pathology and therapeutic targets. Using this informative model for future studies will aid in advancing our knowledge of PAH.

\subsection{Effects of Macitentan on PAH severity}

Macitentan is a novel ERA that works by blocking the negative effects of circulating Endothelin-1, a potent vasoconstrictor and smooth muscle cell mitogen, which has been shown to be upregulated in patients with PAH (Archer et al., 2013; Pisurcik et al., 2013; Lim et al., 2013). ERAs remain to be used as first-line treatment in PAH. Bosentan is one of the most common non-specific ERAs, which has been approved and prescribed since 2001 (Agarwal et al., 2011). Its beneficial effects include improvement in 6-minute walk test and delayed time to clinical worsening. However, the drug fails in improving survival and (Rubin et al., 2002) and is associated with adverse effects that have the potential of causing liver 
injury (Gabbay et al., 2007). The pursuit of discovering an ERA with better efficacy and safety profile has lead to the chemical modification of Bosentan and development of Macitentan. Macitentan has improved tissue distribution and receptor binding affinity and is required in a lower dose (Pulido et al., 2013; Kunita-Takanezawa et al., 2014). The long-term trial SERAPHIN, assessing the efficacy of Macitentan showed improved hemodynamics, a decrease in morbidity and mortality and good safety profile (Pulido et al., 2013).

Chronic treatment with Macitentan in SuHx model was associated with an improvement in PAH severity. This was demonstrated by a significant increase in PAAT and RV EF, parameters of pulmonary pressures and RV function, respectively. Histological findings revealed observably less degree of RV hypertrophy, noticeable regression of occlusive lesions and reduction in medial wall thickening, and fibrosis compared to animals that received no treatment. These results agree with our hypothesis that Macitentan has the potential of improving hemodynamics, pulmonary vascular remodeling and RV function. There was a corresponding decrease in invasive RVSP measurements however the data did not reach significance. The absence of significance may be explained by the varying degree of severity in the development of PAH in the untreated group of rats at week 8 in conjunction with the small sample size, producing high standard deviations within this group. KunitaTakanezawa and colleagues' recent study evaluating the efficacy of Macitentan in a SuHx model showed a mean reduction of RSVP to approximately $45 \mathrm{mmHG}$ following 3 weeks of treatment with Macitentan (Kunita-Takanezawa et al., 2014). The hemodynamic measurements post-Macitentan in our study (RVSP $46.9 \pm 10.7 \mathrm{mmHg}$ at week $8 \mathrm{SuHx}+$ ERA) were very similar to Kinuta-Takanezawa's published data. The pathophysiology of $\mathrm{PAH}$ is known to be a heterogeneous process varying in intensity in people and in animals 
(Jeffery et al., 2002; Archer et al., 2010; Piao et al., 2010). It is possible that there were some animals that were non-responders to the SuHx protocol and that appropriate phenotyping and subsequential stratification of rodents should be carried out in future experiments. Currently there is no published data highlighting the robustness of the SuHx model however, unpublished data from our collaborators found that a subset of rats would not develop severe PAH. As this model has becomes increasingly popular for the study of PAH, it is important to appropriately characterize it and understand its strengths and weaknesses.

Weight ratio measurements demonstrated a trend towards a decrease in RV hypertrophy following Macitentan treatment compared to untreated group, however the data failed to reach significance. We hypothesized that Macitentan treatment would regress RV hypertrophy. As previously mentioned, this discrepancy may be due to the varying degree of PAH induced in the rodents. Another explanation may be improper or inconsistent technical surgical skills when separating the 4 chambers of the heart prior to weighing. Failing to adequately dry the ventricles from blood before weight measurements would have an impact on weight measurements obtained. Histological assessment of RV hypertrophy showed observable less degree of hypertrophy compared to untreated group therefore the discrepancy between these two findings may be due to technical/human error.

The effects mediated by circulating ET-1 include vasoconstriction and platelet-aggregation. An elevation of ET-1 peptide in PAH is a known contributor in the pathogenesis and pulmonary vascular remodeling (Budhiraja et al., 2004). ET-1 is also involved in the regulation of oxygen-dependent transcription factor HIF-1 $\alpha$, a regulator of cellular metabolism (Pisurcik et al., 2013). HIF-1 $\alpha$ is a master regulator of several genes associated 
with cellular contraction, migration and proliferation and codes for various glycolytic proteins (Valle-Casuso et al., 2012). Studies have demonstrated that ET-1 induces HIF-1 $\alpha$ expression and decreases HIF-1 $\alpha$ degradation in PASMC (Pisurcik et al., 2013). In the adult myocardium, ET-1 plays a role in proper coronary blood flow by regulating vascular tone (Drawnell et al., 2012). Elevated levels of ET-1 have been inversely correlated with cardiac function and have been suggested as a marker for future heart failure (Selvais et al., 2000; Zolk et al., 2002). Treatment with endothelin receptor antagonist in our study was associated with a decrease in RV glucose uptake, improved hemodynamics and RV function. Concordant with out hypothesis, this demonstrates that the pathogenic effects of elevated ET-1 may be reversed by treatment with Macitentan. Additionally, this suggests a possible link between the endothelin system and metabolic alterations in PAH. A better understanding of the interplay between ET-1 and metabolism and the development of RHF may lead to a novel diagnostic marker of RHF (Stewart et al., 1991).

\subsection{Cardiac Changes in Metabolism}

Recent human and animal studies show evidence that there is a glycolytic shift in PAH and that these alterations are associated with an activation of glycolytic genes such as GLUT1, PDH and PDK (Oikawa et al., 2005; Fang et al. 2012; Archer et al. 2013) Our findings support this premise as demonstrated by elevated FDG SUVs in the RV and associated upregulation in glucose transporter GLUT4. There was a strong inverse correlation between increased FDG uptake and RV function suggesting that the glycolytic shift is maladaptive. Glycolysis is less efficient at producing ATP than oxidative metabolism and only yields 2ATP as opposed to 36ATP per glucose molecule (Hue et al., 2009). As such, impaired ATP 
generation may not support the cardiac demand to produce adequate contractile power and thus contribute to RV dysfunction. There have been few studies evaluating changes in glucose metabolism serially over time but research suggests that RV glucose uptake increases as the severity of RV dysfunction increases (Archer et al., 2013) Although our research demonstrated a significant increase in RV FDG at week $5 \mathrm{SuHx}$ and week $8 \mathrm{SuHx}$ compared to control, there was no significant progressive increase from week $5 \mathrm{SuHx}$ to week $8 \mathrm{SuHx}$. This may be due to methodological and technical issues. The heterogeneity of disease development may be a reason as to why FDG SUVs showed such variability. Omitting rodents that do not develop severe PAH should be performed in future experiments. Additionally, increasing the number of animals to produce a larger sample size may decrease variability and reveal a more accurate picture of metabolic changes. Another possibility may be explained by physiology. It may be hypothesized that at 5 weeks, the maximum cellular FDG uptake is reached, and hence no measured differences are detected between week 5 and week 8. Performing additional biochemical assays that measure changes in metabolic protein concentrations would be beneficial to better characterize the sequence of metabolic alterations.

Overall RV kinetic analysis of FDG was concordant with the measured RV FDG SUV at 5 weeks and 8 weeks post Sugen, showing only a significant increase at 5 weeks with the onset of PAH. Unexpectedly, rMGU following Macitentan treatment did not match what was observed in FDG SUV data, and showed no significant change with treatment. Kinetic analysis takes into account the metabolic status of the animals at the time of the scan. Measured serum glucose levels were variable within the group of animals and the added factor played a role in augmenting standard deviations. A possible control for this may be to 
fast the animals, or use an insulin-glucose clamp and standardize the metabolic status of the animals (Vitale et al., 2001). Furthermore, an alternative explanation for this discrepancy may be suboptimal source of image derived blood input function used for the generation of Patlak graph. In this study, a region of interest was drawn within the LV cavity to generate the blood input curve. Due to the small size of the cavity, and perhaps the effect of a dilated RV impeding on the left side of the heart, partial volume effects and spillover would produce an overestimation in radioactivity concentration. Thorn described a method for assessing myocardial FDG kinetics using a vena cava image-derived blood input function in a mouse model (Thorn et al., 2013). This may be an alternative way of obtaining more accurate rate of myocardial glucose uptake measurements.

Little is known about the adaptive changes in fatty acid metabolism and the cross-talk between glucose-fatty acid cycle in the right ventricle (Fang et al., 2012). The benefit of therapeutically inhibiting FAO on improving RV function has previously been shown (Fang et al., 2012; Archer et al., 2013). The premise is driven by the Randle cycle, which describes the dynamic and competitive relationship between glucose and FAO. Under these conditions, energy production is rerouted from FAO, which utilizes $12 \%$ more oxygen for yielding ATP to the more metabolically efficient glucose oxidation (Xu et al., 2007). Our study is the first to temporally evaluate fatty acid utilization in vivo with PET imaging in the SuHx rat model of PAH. There was an observed trend towards increased fatty acid uptake with the onset of PAH, and a trend towards elevated RV FTHA PET signals at the week 8 SuHx time point with no treatment, however variability within this group was high, and the changes failed to reach significance. 
The published data exploring changes in fatty acid metabolism in RHF and PAH is conflicting. Certain author's suggest there is a metabolic switch associated with increased FAO in RVH (Sutendra et al., 2010; Fragasso et al., 2012 Fang et al., 2012) or in ischemic heart disease (Dyck et al., 2004) that contributes to reduced contractility and a decline in ventricular function. Other studies however, show a downregulation of fatty acid metabolism during RV hypertrophy and heart failure (Kolwicz Jr. et al., 2011; Gomez-Arroyo et al., 2013). It has been proposed that there is a switch to the fetal gene program, in which a heart under stress reverts to using glycolysis primarily for energy production (Taegtmeyer et al., 2010). In an adaptive response to stress, the heart will reactivate the fetal gene program to try and escape cell death however in the long term this process is detrimental and leads to the decline of heart function (Taegtmeyer et al., 2010). Although our knowledge of changes in fatty acid metabolism is incomplete, the benefit of inhibiting fatty acid metabolism to stimulate glucose oxidation has proven valuable. Fang and colleagues demonstrated the benefits of clinically available metabolic modulators Ranolizine and Trimetazidine, on improving RV function in an animal model of pressure overload (Fang et al., 2011). Lopaschuk and colleagues reviewed the phenomenon of elevated fatty acids following a myocardial ischemic episode and the cardioprotective effects of using an MCD inhibitor to inhibit FAO and stimulate GO (Lopaschuk et al., 2006). Additionally, Lopaschuk's group engineered mice to lack MCD (-/-) and demonstrated that in this model, the animals were able to recover from ischemic events with improved cardiac contractility (Lopaschuk et al., 2006). Studies that show the beneficial effects of inhibiting fatty acid oxidation on RV function provide a novel approach to treating RHF associated with PAH. 
Our findings suggest that there may be a link between increased FAO rates at week $5 \mathrm{SuHx}$ and impaired cardiac performance (Bonnett et al., 2006; Archer et al., 2013). Although the data showed a trend, no significant change in FA uptake was measured throughout the progression of $\mathrm{PAH}$ or following Macitentan. This may again be explained by the heterogeneity of PAH development or by methodological concerns and small sample size. It is also possible that FAO reaches maximal rates at week $5 \mathrm{SuHx}$ and with the development of advanced heart failure, becomes downregulated. Neubauer reviewed the energy deprived failing heart and described the heterogeneity and complexity associated with metabolic changes that the myocardium undergoes (Neubauer et al. 2007). In this review Neubauer cites a few studies describing elevations in FAO during early heart failure, and significantly decreased rates of FAO in advanced heart failure, (Osorio et al., 2002; Chandler et al., 2004; Stanley et al., 2005; Neubauer et al. 2007) agreeing with our preliminary findings. This area of investigation remains rudimentary and characterizing changes in fatty acid oxidation in RHF will add to our understanding of the development of heart failure and provide novel avenues for treatment via metabolic modulation.

There was no change in LV tracer uptake throughout the development of PAH with both FDG and FTHA tracer. This is in line with what is observed in clinical cases of PAH (Oikawa et al., 2005; Lundgrin et al., 2012; unpublished data from our group).

\subsection{Lung Changes in Metabolism}

There is increasing evidence that pathologic remodeling of the pulmonary vasculature is associated with abnormal cellular metabolism and studies with patients and animals have 
shown increased lung uptake with FDG PET (Hagan et al., 2011; Marsboom et al., 2012; Zhao et al., 2013; Ryan et al., 2013) Two studies using the monocrotaline rat model of severe PAH have shown that increased accumulation of FDG tracer may be detected in lung parenchyma and that there is an associated increased expression of GLUT1 and other glycolytic proteins ( McLaughlin et al., 2004; Zhao et al., 2013) Marsboom's group demonstrated that elevated lung FDG signals may be detected during early, mild PAH and that the signal progressively increases with evolving PAH. Both groups also showed that lung FDG PET was sensitive enough to detect the therapeutic benefit of drugs. In contrast to Zhao and Marsboom, we did not find any changes in lung FDG uptake throughout the time course of PAH development or in response to therapy. Additionally, no changes were observed with FTHA tracer. This discord may be explained by differences in the animal models. Monocrotaline is a toxic substance that induces endothelial damage and is associated with inflammation (Rubin et al., 2002). PET imaging with FDG tracer has been used to image several diseases associated with inflammation due to its accelerated accumulation within inflamed tissue (Miyagawa et al., 2014). It could be possible that elevated lung FDG uptake in the MCT model may be in part due to the inflammatory response to the toxic substance. Marsboom however, did use the SuHx model to validate lung FDG PET imaging for detecting metabolic changes and identified accelerated glucose uptake within the tissue. More studies serially evaluating glucose uptake in both humans and experimental PAH are warranted.

\subsection{Study Limitations}


A limitation to using FDG PET to study and measure changes in glucose metabolism is presented by fact that FDG does not distinguish between glycolysis and glucose oxidation. Therefore, careful interpretation when using metabolic imaging is needed, and alternate ways of validating results should be carried out in preclinical studies. The increase in GLUT 4 expression found in this study supports the hypothesis that the increased FDG uptake represents increased glycolysis. One advantage to using PET for research is that it can be used serially and non-invasively, and thus it represents a useful tool for translation into clinical studies. Translation to clinical studies will allow us to gain further insight into mechanisms of disease as well as evaluate the role of novel imaging in the diagnosis and management of disease. Another limitation to using PET imaging is partial volume effect (PVE). This technical issue may decrease quantitative accuracy and is a result of limited resolution of the imaging device causing regional values to be under-estimated. This becomes specifically important when dealing with small structures such as the heart walls. To correct for this in the future, a secondary imaging modality such as computed tomography (CT) or magnetic resonance imaging (MRI) may be used to obtain accurate anatomical reference, which may properly correct the regional values (Su et al., 2009). Image derived blood input function may also be subject to PVE due to small cavity size and therefore may bias the kinetic analysis results. For a more reliable means of kinetic analysis, manual blood collection during the time of scan would provide the most accurate input function however this methodology is invasive and does not typically permit serial imaging in the same animal.

Due to small sample size and variability, changes in invasively measured right ventricular systolic pressures failed to reach significance in this study. Despite this, we were able to demonstrate significant metabolic change with PET imaging, vascular remodeling on 
histology and improvements with Macitentan therapy. In future studies, phenotyping the rodents at an early timepoint and eliminating non-responders will ensure that the rats being carried through to subsequent evaluations have developed severe PAH. By stratifying the animals, this may lower variability within the group and allow to differentiate between adaptive, non-adaptive, mild and severe pathophysiology of $\mathrm{PAH}$.

Conducting numerous serial in-vivo evaluations requiring anesthesia in the animals caused

Metabolic changes were not observed with lung PET imaging. This may be a limitation of the SuHx model as discussed previously or may represent true heterogeneity in lung metabolism in PAH, especially in small animal models. There are limited studies evaluating changes in lung metabolism, and most of the published results have been investigated in MCT rat model. It has previously been demonstrated that lung FDG uptake is heterogeneous in clinical patients (Zhao et al., 2013).

Macitentan resulted in a significant decrease in RV FDG uptake corresponding to an improvement in RV function and reduction of PAH severity. Whether Macitentan had a direct effect on improving RV energetics through ET-1 receptor blockade or indirectly through the successful treatment of PAH (and subsequent RV afterload) cannot be determined from this study. Future studies demonstrating the effects of Macitentan in PAH independent models of RV failure (such as pulmonary artery banding) are required. 


\subsection{CONCLUSIONS AND FUTURE WORK}

In conclusion SuHx induced $\mathrm{PAH}$, a model of maladaptive $\mathrm{RVH}$, is associated with metabolic changes in the RV, characterized by increased glucose uptake and a trends towards increased fatty acid uptake. Macitentan attenuated RV FDG uptake and significantly increased RV function as well as showed trends towards lowered hemodynamics and regressed vascular remodeling compared to untreated group.

The results of this study has advanced our understanding of metabolic alterations and the pathogenesis of right HF; data which is currently lacking in this field. In addition, this study demonstrates a role for metabolic imaging as an in-vivo tool for the study of cardiac metabolism and has the potential for further translational study in humans. This is a vital initial step towards further research in the role of metabolic modulators in the treatment and prevention of right $\mathrm{HF}$ in PAH and may have future implications for the growing patient population with other forms of right HF. The long-term goal is to evaluate whether metabolic modulation may be used in the prevention and treatment of right HF. In order to achieve this goal several fundamental questions have been addressed with this project and subsequent studies using novel in-vivo imaging techniques are currently underway. 


\subsection{REFERENCES}

Abe, K., Toba, M., Alzoubi, A., Ito, M., Fagan, K. A., Cool, C. D., . . . Oka, M. (2010). Formation of plexiform lesions in experimental severe pulmonary arterial hypertension. Circulation, 121(25), 2747-2754.

Agarwal, R., \& Gomberg-Maitland, M. (2011). Current therapeutics and practical management strategies for pulmonary arterial hypertension. Am Heart J, 162(2), 201213.

Archer, S. L. (2013). Mitochondrial dynamics--mitochondrial fission and fusion in human diseases. N Engl J Med, 369(23), 2236-2251.

Archer, S. L., Weir, E. K., \& Wilkins, M. R. (2010). Basic science of pulmonary arterial hypertension for clinicians: new concepts and experimental therapies. Circulation, 121(18), 2045-2066.

Bauer, N. R., Moore, T. M., \& McMurtry, I. F. (2007). Rodent models of PAH: are we there yet? Am J Physiol Lung Cell Mol Physiol, 293(3), L580-582.

Bogaard, H. J., Abe, K., Vonk Noordegraaf, A., \& Voelkel, N. F. (2009). The right ventricle under pressure: cellular and molecular mechanisms of right-heart failure in pulmonary hypertension. Chest, 135(3), 794-804.

Bogaard, H. J., Natarajan, R., Henderson, S. C., Long, C. S., Kraskauskas, D., Smithson, L., . . . Voelkel, N. F. (2009). Chronic pulmonary artery pressure elevation is insufficient to explain right heart failure. Circulation, 120(20), 1951-1960.

Bonnet, S., Michelakis, E. D., Porter, C. J., Andrade-Navarro, M. A., Thebaud, B., Bonnet, S., . . . Archer, S. L. (2006). An abnormal mitochondrial-hypoxia inducible factorlalpha-Kv channel pathway disrupts oxygen sensing and triggers pulmonary arterial hypertension in fawn hooded rats: similarities to human pulmonary arterial hypertension. Circulation, 113(22), 2630-2641.

Budhiraja, R., Tuder, R. M., \& Hassoun, P. M. (2004). Endothelial dysfunction in pulmonary hypertension. Circulation, 109(2), 159-165.

Campo, A., Mathai, S. C., Le Pavec, J., Zaiman, A. L., Hummers, L. K., Boyce, D., . . . Hassoun, P. M. (2011). Outcomes of hospitalisation for right heart failure in pulmonary arterial hypertension. Eur Respir J, 38(2), 359-367. 
Chakinala, M. M., \& Barst, R. (2013). From short-term benefits to long-term outcomes: the evolution of clinical trials in pulmonary arterial hypertension. Pulm Circ, 3(3), 507522.

Chandler, M. P., Kerner, J., Huang, H., Vazquez, E., Reszko, A., Martini, W. Z., . . Stanley, W. C. (2004). Moderate severity heart failure does not involve a downregulation of myocardial fatty acid oxidation. Am J Physiol Heart Circ Physiol, 287(4), H15381543.

Clozel, M., Hess, P., Rey, M., Iglarz, M., Binkert, C., \& Qiu, C. (2006). Bosentan, sildenafil, and their combination in the monocrotaline model of pulmonary hypertension in rats. Exp Biol Med (Maywood), 231(6), 967-973.

Crosswhite, P., \& Sun, Z. (2014). Molecular mechanisms of pulmonary arterial remodeling. Mol Med, 20, 191-201.

Dewachter, L., Dewachter, C., \& Naeije, R. (2010). New therapies for pulmonary arterial hypertension: an update on current bench to bedside translation. Expert Opin Investig Drugs, 19(4), 469-488.

Drake, J. M. (2010). Shunt failure. J Neurosurg Pediatr, 6(4), 316-317; discussion 317.

Drawnel, F. M., Archer, C. R., \& Roderick, H. L. (2013). The role of the paracrine/autocrine mediator endothelin-1 in regulation of cardiac contractility and growth. $\mathrm{Br} J$ Pharmacol, 168(2), 296-317.

Dyck, J. R., \& Lopaschuk, G. D. (2006). AMPK alterations in cardiac physiology and pathology: enemy or ally? J Physiol, 574(Pt 1), 95-112.

Fang, Y. H., Piao, L., Hong, Z., Toth, P. T., Marsboom, G., Bache-Wiig, P., . . Archer, S. L. (2012). Therapeutic inhibition of fatty acid oxidation in right ventricular hypertrophy: exploiting Randle's cycle. J Mol Med (Berl), 90(1), 31-43.

Farber, H. W., \& Loscalzo, J. (2005). Mechanism of disease: Pulmonary hypertension. Discov Med, 5(25), 80-87.

Fessel, J. P., Flynn, C. R., Robinson, L. J., Penner, N. L., Gladson, S., Kang, C. J., . . West, J. D. (2013). Hyperoxia synergizes with mutant bone morphogenic protein receptor 2 to cause metabolic stress, oxidant injury, and pulmonary hypertension. Am J Respir Cell Mol Biol, 49(5), 778-787.

Fessel, J. P., Loyd, J. E., \& Austin, E. D. (2011). The genetics of pulmonary arterial hypertension in the post-BMPR2 era. Pulm Circ, 1(3), 305-319.

Fijalkowska, I., Xu, W., Comhair, S. A., Janocha, A. J., Mavrakis, L. A., Krishnamachary, B., . . . Tuder, R. M. (2010). Hypoxia inducible-factor1alpha regulates the metabolic shift of pulmonary hypertensive endothelial cells. Am J Pathol, 176(3), 1130-1138. 
Fragasso, G., Rosano, G., Baek, S. H., Sisakian, H., Di Napoli, P., Alberti, L., . . . Belardinelli, R. (2013). Effect of partial fatty acid oxidation inhibition with trimetazidine on mortality and morbidity in heart failure: results from an international multicentre retrospective cohort study. Int J Cardiol, 163(3), 320-325.

Gabbay, E., Fraser, J., \& McNeil, K. (2007). Review of bosentan in the management of pulmonary arterial hypertension. Vasc Health Risk Manag, 3(6), 887-900.

Galie, N., Manes, A., \& Branzi, A. (2004a). The endothelin system in pulmonary arterial hypertension. Cardiovasc Res, 61(2), 227-237.

Galie, N., Manes, A., \& Branzi, A. (2004b). Evaluation of pulmonary arterial hypertension. Curr Opin Cardiol, 19(6), 575-581.

Galie, N., Manes, A., \& Branzi, A. (2004c). New insights on pulmonary arterial hypertension. Rev Esp Cardiol (Engl Ed), 57(7), 603-607.

Ginghina, C., Muraru, D., Vladaia, A., Jurcut, R., Popescu, B. A., Calin, A., \& Giusca, S. (2009). Doppler flow patterns in the evaluation of pulmonary hypertension. Rom $J$ Intern Med, 47(2), 109-121.

Gomez-Arroyo, J., Mizuno, S., Szczepanek, K., Van Tassell, B., Natarajan, R., dos Remedios, C. G., . . . Voelkel, N. F. (2013). Metabolic gene remodeling and mitochondrial dysfunction in failing right ventricular hypertrophy secondary to pulmonary arterial hypertension. Circ Heart Fail, 6(1), 136-144.

Greyson, C. R. (2010). The right ventricle and pulmonary circulation: basic concepts. Rev Esp Cardiol (Engl Ed), 63(1), 81-95. doi: 10.1016/S1885-5857(10)70012-8

Guignabert, C., \& Dorfmuller, P. (2013). Pathology and pathobiology of pulmonary hypertension. Semin Respir Crit Care Med, 34(5), 551-559.

Guignabert, C., Izikki, M., Tu, L. I., Li, Z., Zadigue, P., Barlier-Mur, A. M., . . Eddahibi, S. (2006). Transgenic mice overexpressing the 5-hydroxytryptamine transporter gene in smooth muscle develop pulmonary hypertension. Circ Res, 98(10), 1323-1330.

Guo, H., Bao, Z., Li, J., Lian, S., Wang, S., He, Y., . . . Hu, X. (2012). Molecular characterization of TGF-beta type I receptor gene (Tgfbr1) in Chlamys farreri, and the association of allelic variants with growth traits. PLoS One, 7(11), e51005.

Hagan, G., Southwood, M., Treacy, C., Ross, R. M., Soon, E., Coulson, J., . . . Rudd, J. H. (2011). (18)FDG PET imaging can quantify increased cellular metabolism in pulmonary arterial hypertension: A proof-of-principle study. Pulm Circ, 1(4), 448455. 
Heath, D., Smith, P., Gosney, J., Mulcahy, D., Fox, K., Yacoub, M., \& Harris, P. (1987). The pathology of the early and late stages of primary pulmonary hypertension. Br Heart $J$, 58(3), 204-213.

Hue, L., \& Taegtmeyer, H. (2009). The Randle cycle revisited: a new head for an old hat. Am J Physiol Endocrinol Metab, 297(3), E578-591.

Humbert, M., Montani, D., Perros, F., Dorfmuller, P., Adnot, S., \& Eddahibi, S. (2008). Endothelial cell dysfunction and cross talk between endothelium and smooth muscle cells in pulmonary arterial hypertension. Vascul Pharmacol, 49(4-6), 113-118.

Humbert, M., Morrell, N. W., Archer, S. L., Stenmark, K. R., MacLean, M. R., Lang, I. M., . . Rabinovitch, M. (2004). Cellular and molecular pathobiology of pulmonary arterial hypertension. J Am Coll Cardiol, 43(12 Suppl S), 13S-24S.

Jeffery, T. K., \& Morrell, N. W. (2002). Molecular and cellular basis of pulmonary vascular remodeling in pulmonary hypertension. Prog Cardiovasc Dis, 45(3), 173-202.

Jonigk, D., Golpon, H., Bockmeyer, C. L., Maegel, L., Hoeper, M. M., Gottlieb, J., . . . Laenger, F. (2011). Plexiform lesions in pulmonary arterial hypertension composition, architecture, and microenvironment. Am J Pathol, 179(1), 167-179.

Kolwicz, S. C., Jr., \& Tian, R. (2011). Glucose metabolism and cardiac hypertrophy. Cardiovasc Res, 90(2), 194-201.

Koskenvuo, J. W., Mirsky, R., Zhang, Y., Angeli, F. S., Jahn, S., Alastalo, T. P., . . . Yeghiazarians, Y. (2010). A comparison of echocardiography to invasive measurement in the evaluation of pulmonary arterial hypertension in a rat model. Int $J$ Cardiovasc Imaging, 26(5), 509-518.

Kunita-Takanezawa, M., Abe, K., Hirooka, Y., Kuwabara, Y., Hirano, K., Oka, M., \& Sunagawa, K. (2014). Novel Dual Endothelin Receptor Antagonist Macitentan Reverses Severe Pulmonary Arterial Hypertension in Rats. J Cardiovasc Pharmacol.

Lang, M., Kojonazarov, B., Tian, X., Kalymbetov, A., Weissmann, N., Grimminger, F., . . Schermuly, R. T. (2012). The soluble guanylate cyclase stimulator riociguat ameliorates pulmonary hypertension induced by hypoxia and SU5416 in rats. PLoS One, 7(8), e43433.

Lim, C. S., Kiriakidis, S., Sandison, A., Paleolog, E. M., \& Davies, A. H. (2013). Hypoxiainducible factor pathway and diseases of the vascular wall. J Vasc Surg, 58(1), 219230.

Lopaschuk, G. D., \& Stanley, W. C. (2006). Malonyl-CoA decarboxylase inhibition as a novel approach to treat ischemic heart disease. Cardiovasc Drugs Ther, 20(6), 433439. 
Lopaschuk, G. D., Ussher, J. R., Folmes, C. D., Jaswal, J. S., \& Stanley, W. C. (2010). Myocardial fatty acid metabolism in health and disease. Physiol Rev, 90(1), 207-258.

Marsboom, G., Wietholt, C., Haney, C. R., Toth, P. T., Ryan, J. J., Morrow, E., . . Archer, S. L. (2012). Lung (1)(8)F-fluorodeoxyglucose positron emission tomography for diagnosis and monitoring of pulmonary arterial hypertension. Am J Respir Crit Care Med, 185(6), 670-679.

McGoon, M. D., \& Kane, G. C. (2009). Pulmonary hypertension: diagnosis and management. Mayo Clin Proc, 84(2), 191-207.

McLaughlin, V. V. (2004). Classification and epidemiology of pulmonary hypertension. Cardiol Clin, 22(3), 327-341, v.

McLaughlin, V. V., Davis, M., \& Cornwell, W. (2011). Pulmonary arterial hypertension. Curr Probl Cardiol, 36(12), 461-517.

McLaughlin, V. V., \& Rich, S. (2004). Pulmonary hypertension. Curr Probl Cardiol, 29(10), 575-634.

McMurtry, M. S., Bonnet, S., Wu, X., Dyck, J. R., Haromy, A., Hashimoto, K., \& Michelakis, E. D. (2004). Dichloroacetate prevents and reverses pulmonary hypertension by inducing pulmonary artery smooth muscle cell apoptosis. Circ Res, 95(8), 830-840.

Michelakis, E. D. (2014). Pulmonary arterial hypertension: yesterday, today, tomorrow. Circ Res, 115(1), 109-114.

Miyagawa, K., \& Emoto, N. (2014). Current state of endothelin receptor antagonism in hypertension and pulmonary hypertension. Ther Adv Cardiovasc Dis, 8(5), 202-216.

Morimatsu, Y., Sakashita, N., Komohara, Y., Ohnishi, K., Masuda, H., Dahan, D., . . . Marthan, R. (2012). Development and characterization of an animal model of severe pulmonary arterial hypertension. J Vasc Res, 49(1), 33-42.

Morrell, N. W., Archer, S. L., Defelice, A., Evans, S., Fiszman, M., Martin, T., . . . Stenmark, K. R. (2013). Anticipated classes of new medications and molecular targets for pulmonary arterial hypertension. Pulm Circ, 3(1), 226-244.

Nagendran, J., Archer, S. L., Soliman, D., Gurtu, V., Moudgil, R., Haromy, A., . . . Michelakis, E. D. (2007). Phosphodiesterase type 5 is highly expressed in the hypertrophied human right ventricle, and acute inhibition of phosphodiesterase type 5 improves contractility. Circulation, 116(3), 238-248.

Nassiri, F., Cusimano, M. D., Scheithauer, B. W., Rotondo, F., Fazio, A., Yousef, G. M., .. . Lloyd, R. V. (2011). Endoglin (CD105): a review of its role in angiogenesis and tumor diagnosis, progression and therapy. Anticancer Res, 31(6), 2283-2290. 
Neubauer, S. (2007). The failing heart--an engine out of fuel. N Engl J Med, 356(11), 11401151.

Nicolls, M. R., Mizuno, S., Taraseviciene-Stewart, L., Farkas, L., Drake, J. I., Al Husseini, A., . . . Bogaard, H. J. (2012). New models of pulmonary hypertension based on VEGF receptor blockade-induced endothelial cell apoptosis. Pulm Circ, 2(4), 434442.

Nishimura, T., Faul, J. L., Berry, G. J., Vaszar, L. T., Qiu, D., Pearl, R. G., \& Kao, P. N. (2002). Simvastatin attenuates smooth muscle neointimal proliferation and pulmonary hypertension in rats. Am J Respir Crit Care Med, 166(10), 1403-1408.

Obrzut, S., Jamshidi, N., Karimi, A., Birgersdotter-Green, U., \& Hoh, C. (2010). Imaging and modeling of myocardial metabolism. J Cardiovasc Transl Res, 3(4), 384-396.

Oikawa, M., Kagaya, Y., Otani, H., Sakuma, M., Demachi, J., Suzuki, J., . . . Shirato, K. (2005). Increased [18F]fluorodeoxyglucose accumulation in right ventricular free wall in patients with pulmonary hypertension and the effect of epoprostenol. $\mathrm{J} \mathrm{Am}$ Coll Cardiol, 45(11), 1849-1855.

Osorio, J. C., Stanley, W. C., Linke, A., Castellari, M., Diep, Q. N., Panchal, A. R., . . . Recchia, F. A. (2002). Impaired myocardial fatty acid oxidation and reduced protein expression of retinoid $\mathrm{X}$ receptor-alpha in pacing-induced heart failure. Circulation, 106(5), 606-612.

Pardali, E., \& Ten Dijke, P. (2012). TGFbeta signaling and cardiovascular diseases. Int J Biol Sci, 8(2), 195-213.

Peacock, A. J., Murphy, N. F., McMurray, J. J., Caballero, L., \& Stewart, S. (2007). An epidemiological study of pulmonary arterial hypertension. Eur Respir J, 30(1), 104109.

Phillips, P. G., Long, L., Wilkins, M. R., \& Morrell, N. W. (2005). cAMP phosphodiesterase inhibitors potentiate effects of prostacyclin analogs in hypoxic pulmonary vascular remodeling. Am J Physiol Lung Cell Mol Physiol, 288(1), L103-115.

Piao, L., Fang, Y. H., Cadete, V. J., Wietholt, C., Urboniene, D., Toth, P. T., . . Archer, S. L. (2010). The inhibition of pyruvate dehydrogenase kinase improves impaired cardiac function and electrical remodeling in two models of right ventricular hypertrophy: resuscitating the hibernating right ventricle. J Mol Med (Berl), 88(1), 47-60.

Piao, L., Marsboom, G., \& Archer, S. L. (2010). Mitochondrial metabolic adaptation in right ventricular hypertrophy and failure. J Mol Med (Berl), 88(10), 1011-1020. 
Pietra, G. G., Edwards, W. D., Kay, J. M., Rich, S., Kernis, J., Schloo, B., . . et al. (1989). Histopathology of primary pulmonary hypertension. A qualitative and quantitative study of pulmonary blood vessels from 58 patients in the National Heart, Lung, and Blood Institute, Primary Pulmonary Hypertension Registry. Circulation, 80(5), 11981206.

Pisarcik, S., Maylor, J., Lu, W., Yun, X., Undem, C., Sylvester, J. T., ... Shimoda, L. A. (2013). Activation of hypoxia-inducible factor-1 in pulmonary arterial smooth muscle cells by endothelin-1. Am J Physiol Lung Cell Mol Physiol, 304(8), L549-561.

Price, L. C., Wort, S. J., Finney, S. J., Marino, P. S., \& Brett, S. J. (2010). Pulmonary vascular and right ventricular dysfunction in adult critical care: current and emerging options for management: a systematic literature review. Crit Care, 14(5), R169.

Pulido, T., Adzerikho, I., Channick, R. N., Delcroix, M., Galie, N., Ghofrani, H. A., . . . Investigators, S. (2013). Macitentan and morbidity and mortality in pulmonary arterial hypertension. $N$ Engl J Med, 369(9), 809-818.

Rabinovitch, M. (2004). The mouse through the looking glass: a new door into the pathophysiology of pulmonary hypertension. Circ Res, 94(8), 1001-1004.

Rabinovitch, M. (2012). Molecular pathogenesis of pulmonary arterial hypertension. J Clin Invest, 122(12), 4306-4313.

Rai, P. R., Cool, C. D., King, J. A., Stevens, T., Burns, N., Winn, R. A., . . Voelkel, N. F. (2008). The cancer paradigm of severe pulmonary arterial hypertension. Am J Respir Crit Care Med, 178(6), 558-564.

Raja, S. G., \& Dreyfus, G. D. (2008). Current status of bosentan for treatment of pulmonary hypertension. Ann Card Anaesth, 11(1), 6-14.

Remillard, C. V., Tigno, D. D., Platoshyn, O., Burg, E. D., Brevnova, E. E., Conger, D., . . . Yuan, J. X. (2007). Function of Kv1.5 channels and genetic variations of KCNA5 in patients with idiopathic pulmonary arterial hypertension. Am J Physiol Cell Physiol, 292(5), C1837-1853.

Roth, R. A., Dotzlaf, L. A., Baranyi, B., Kuo, C. H., \& Hook, J. B. (1981). Effect of monocrotaline ingestion on liver, kidney, and lung of rats. Toxicol Appl Pharmacol, 60(2), 193-203.

Ruan, C. H., Dixon, R. A., Willerson, J. T., \& Ruan, K. H. (2010). Prostacyclin therapy for pulmonary arterial hypertension. Tex Heart Inst J, 37(4), 391-399.

Rubin, L. J., Badesch, D. B., Barst, R. J., Galie, N., Black, C. M., Keogh, A., . . Simonneau, G. (2002). Bosentan therapy for pulmonary arterial hypertension. $N$ Engl J Med, 346(12), 896-903. 
Ryan, J. J., \& Archer, S. L. (2014). The right ventricle in pulmonary arterial hypertension: disorders of metabolism, angiogenesis and adrenergic signaling in right ventricular failure. Circ Res, 115(1), 176-188.

Sakao, S., Tatsumi, K., \& Voelkel, N. F. (2009). Endothelial cells and pulmonary arterial hypertension: apoptosis, proliferation, interaction and transdifferentiation. Respir Res, 10, 95. doi: 10.1186/1465-9921-10-95

Sakao, S., Tatsumi, K., \& Voelkel, N. F. (2010). Reversible or irreversible remodeling in pulmonary arterial hypertension. Am J Respir Cell Mol Biol, 43(6), 629-634.

Schermuly, R. T., Kreisselmeier, K. P., Ghofrani, H. A., Yilmaz, H., Butrous, G., Ermert, L., .. . Grimminger, F. (2004). Chronic sildenafil treatment inhibits monocrotalineinduced pulmonary hypertension in rats. Am J Respir Crit Care Med, 169(1), 39-45.

Sebkhi, A., Strange, J. W., Phillips, S. C., Wharton, J., \& Wilkins, M. R. (2003). Phosphodiesterase type 5 as a target for the treatment of hypoxia-induced pulmonary hypertension. Circulation, 107(25), 3230-3235.

Selvais, P. L., Robert, A., Ahn, S., van Linden, F., Ketelslegers, J. M., Pouleur, H., \& Rousseau, M. F. (2000). Direct comparison between endothelin-1, N-terminal proatrial natriuretic factor, and brain natriuretic peptide as prognostic markers of survival in congestive heart failure. J Card Fail, 6(3), 201-207.

Simonneau, G. (2008). [Pulmonary hypertension. Major therapeutic advances]. Rev Prat, 58(18), 1989-1990.

Soubrier, F., Chung, W. K., Machado, R., Grunig, E., Aldred, M., Geraci, M., .. . Humbert, M. (2013). Genetics and genomics of pulmonary arterial hypertension. J Am Coll Cardiol, 62(25 Suppl), D13-21.

Stanley, W. C., \& Sabbah, H. N. (2005). Metabolic therapy for ischemic heart disease: the rationale for inhibition of fatty acid oxidation. Heart Fail Rev, 10(4), 275-279.

Stasch, J. P., Pacher, P., \& Evgenov, O. V. (2011). Soluble guanylate cyclase as an emerging therapeutic target in cardiopulmonary disease. Circulation, 123(20), 2263-2273.

Steele, P., Strange, G., Wlodarczyk, J., Dalton, B., Stewart, S., Gabbay, E., \& Keogh, A. (2010). Hemodynamics in pulmonary arterial hypertension (PAH): do they explain long-term clinical outcomes with PAH-specific therapy? BMC Cardiovasc Disord, 10,9 .

Stenmark, K. R., Meyrick, B., Galie, N., Mooi, W. J., \& McMurtry, I. F. (2009). Animal models of pulmonary arterial hypertension: the hope for etiological discovery and pharmacological cure. Am J Physiol Lung Cell Mol Physiol, 297(6), L1013-1032. 
Stewart, D. J., Levy, R. D., Cernacek, P., \& Langleben, D. (1991). Increased plasma endothelin-1 in pulmonary hypertension: marker or mediator of disease? Ann Intern Med, 114(6), 464-469.

Su, K. H., Lee, J. S., Li, J. H., Yang, Y. W., Liu, R. S., \& Chen, J. C. (2009). Partial volume correction of the microPET blood input function using ensemble learning independent component analysis. Phys Med Biol, 54(6), 1823-1846.

Sutendra, G., Bonnet, S., Rochefort, G., Haromy, A., Folmes, K. D., Lopaschuk, G. D., . . . Michelakis, E. D. (2010). Fatty acid oxidation and malonyl-CoA decarboxylase in the vascular remodeling of pulmonary hypertension. Sci Transl Med, 2(44), 44ra58.

Taegtmeyer, H., Sen, S., \& Vela, D. (2010). Return to the fetal gene program: a suggested metabolic link to gene expression in the heart. Ann N Y Acad Sci, 1188, 191-198.

Taraseviciene-Stewart, L., Kasahara, Y., Alger, L., Hirth, P., Mc Mahon, G., Waltenberger, J., . . Tuder, R. M. (2001). Inhibition of the VEGF receptor 2 combined with chronic hypoxia causes cell death-dependent pulmonary endothelial cell proliferation and severe pulmonary hypertension. FASEB J, 15(2), 427-438.

Thorn, S. L., deKemp, R. A., Dumouchel, T., Klein, R., Renaud, J. M., Wells, R. G., . . . DaSilva, J. N. (2013). Repeatable noninvasive measurement of mouse myocardial glucose uptake with 18F-FDG: evaluation of tracer kinetics in a type 1 diabetes model. J Nucl Med, 54(9), 1637-1644.

Upton, P. D., \& Morrell, N. W. (2013). The transforming growth factor-beta-bone morphogenetic protein type signalling pathway in pulmonary vascular homeostasis and disease. Exp Physiol, 98(8), 1262-1266.

Ussher, J. R., \& Lopaschuk, G. D. (2009). Targeting malonyl CoA inhibition of mitochondrial fatty acid uptake as an approach to treat cardiac ischemia/reperfusion. Basic Res Cardiol, 104(2), 203-210.

Valle-Casuso, J. C., Gonzalez-Sanchez, A., Medina, J. M., \& Tabernero, A. (2012). HIF-1 and c-Src mediate increased glucose uptake induced by endothelin-1 and connexin43 in astrocytes. PLoS One, 7(2), e32448.

Van Genechten, J., Brouns, I., Scheuermann, D. W., Timmermans, J. P., \& Adriaensen, D. (2003). Reduced number of intrinsic pulmonary nitrergic neurons in Fawn-Hooded rats as compared to control rat strains. Anat Rec A Discov Mol Cell Evol Biol, 272(1), 446-453.

van Wolferen, S. A., Marcus, J. T., Westerhof, N., Spreeuwenberg, M. D., Marques, K. M., Bronzwaer, J. G., . . . Vonk-Noordegraaf, A. (2008). Right coronary artery flow impairment in patients with pulmonary hypertension. Eur Heart J, 29(1), 120-127.

Vitale, G. D., deKemp, R. A., Ruddy, T. D., Williams, K., \& Beanlands, R. S. (2001). Myocardial glucose utilization and optimization of (18)F-FDG PET imaging in 
patients with non-insulin-dependent diabetes mellitus, coronary artery disease, and left ventricular dysfunction. $J$ Nucl Med, 42(12), 1730-1736.

Voelkel, N. F., Gomez-Arroyo, J., Abbate, A., Bogaard, H. J., \& Nicolls, M. R. (2012). Pathobiology of pulmonary arterial hypertension and right ventricular failure. Eur Respir J, 40(6), 1555-1565.

Watabe, H., Ikoma, Y., Kimura, Y., Naganawa, M., \& Shidahara, M. (2006). PET kinetic analysis--compartmental model. Ann Nucl Med, 20(9), 583-588.

White, R. J., Meoli, D. F., Swarthout, R. F., Kallop, D. Y., Galaria, II, Harvey, J. L., . . . Taubman, M. B. (2007). Plexiform-like lesions and increased tissue factor expression in a rat model of severe pulmonary arterial hypertension. Am J Physiol Lung Cell Mol Physiol, 293(3), L583-590.

Xu, W., Koeck, T., Lara, A. R., Neumann, D., DiFilippo, F. P., Koo, M., . . Erzurum, S. C. (2007). Alterations of cellular bioenergetics in pulmonary artery endothelial cells. Proc Natl Acad Sci U S A, 104(4), 1342-1347.

Zhao, L., Ashek, A., Wang, L., Fang, W., Dabral, S., Dubois, O., . . Wilkins, M. R. (2013). Heterogeneity in lung (18)FDG uptake in pulmonary arterial hypertension: potential of dynamic (18)FDG positron emission tomography with kinetic analysis as a bridging biomarker for pulmonary vascular remodeling targeted treatments. Circulation, 128(11), 1214-1224.

Zolk, O., Quattek, J., Seeland, U., El-Armouche, A., Eschenhagen, T., \& Bohm, M. (2002). Activation of the cardiac endothelin system in left ventricular hypertrophy before onset of heart failure in TG(mREN2)27 rats. Cardiovasc Res, 53(2), 363-371. 